\title{
Supporting Information for Reversible Intramolecular Cycloaddition of Phosphaalkene to an Arene Ring
}

\author{
Liu Leo Liu, Jiliang Zhou, Levy L. Cao, Youngsuk Kim and Douglas W. Stephan* \\ Email: dstephan@chem.utoronto.ca
}

\section{Contents:}

1. General and Synthesis ----------------------------------------------------------------S2-6

2. Copies of NMR Spectra ---

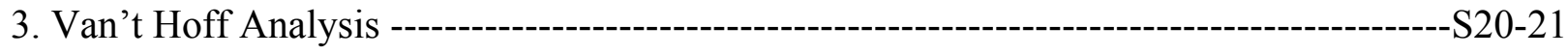

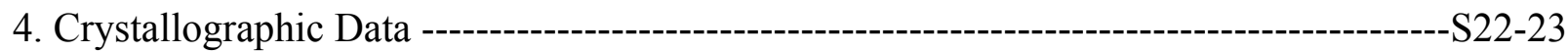

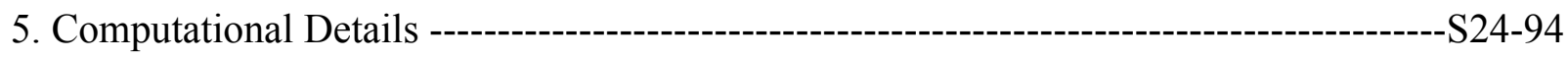

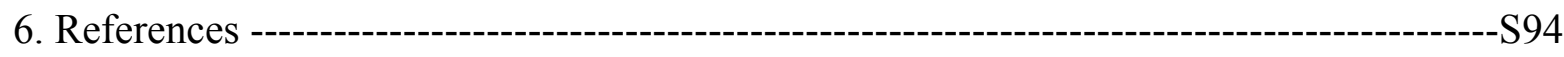




\section{General and Synthesis}

\section{Experimental Section}

General Considerations. All manipulations were performed under an atmosphere of dry, oxygen-free $\mathrm{N}_{2}$ by means of standard Schlenk or glovebox techniques (MBraun LABmaster SP drybox and Innovation Technology glovebox both equipped with a $-35^{\circ} \mathrm{C}$ freezer). Pentane was collected from a Grubbs-type column system manufactured by Innovative Technology. Benzene, $\mathrm{FC}_{6} \mathrm{H}_{5}, \mathrm{ClC}_{6} \mathrm{H}_{5}$ and 1,2-difluorobenzene were dried over $4 \AA$ molecular sieves. Molecular sieves, type $4 \AA$ (pellets, $3.2 \mathrm{~mm}$ diameter) purchased from Sigma-Aldrich were activated prior to usage by iteratively heating with $1050 \mathrm{~W}$ Haier microwave for $5 \mathrm{~min}$ and cooling under vacuo. The process was repeated until no further moisture was released upon heating. $\mathrm{CDCl}_{3}$ and $\mathrm{C}_{6} \mathrm{D}_{6}$, purchased from Cambridge Isotope Laboratories, was degassed and stored over $4 \AA$ molecular sieves in the glovebox for at least $8 \mathrm{~h}$ prior to use. KHMDS, $\mathrm{S}_{8}$ and MeOTf were purchased and used directly. $\operatorname{IPr}^{\mathrm{S} 1}$ and salts $\mathbf{1}$ and $\mathbf{3}^{\mathrm{S} 2}$ were prepared using literature methods. Spectra were recorded on an Agilent DD2 $400 \mathrm{MHz}$, a Bruker Avance III $400 \mathrm{MHz}$ or an Agilent DD2 600 $\mathrm{MHz}$ spectrometer and spectra were referenced to the residual solvent of $\mathrm{CDCl}_{3}\left({ }^{1} \mathrm{H}=7.26 \mathrm{ppm}\right.$; $\left.{ }^{13} \mathrm{C}=77.0 \mathrm{ppm}\right)$ and $\mathrm{C}_{6} \mathrm{D}_{6}\left({ }^{1} \mathrm{H}=7.15 \mathrm{ppm} ;{ }^{13} \mathrm{C}=128.0 \mathrm{ppm}\right)$. Chemical shifts $(\delta)$ are reported in ppm and the absolute values of the coupling constants $(J)$ are in Hz. High resolution mass spectra (HRMS) were obtained on a JMS-T100LC JEOL DART or AB Sciex QStarXL ESI.

Snythsis of 2. Method A: $\mathrm{C}_{6} \mathrm{H}_{6}(3 \mathrm{~mL})$ was added into a mixture of KHMDS (6.1 mg, 0.0305 $\mathrm{mmol})$ and 1 (20 mg, $0.0305 \mathrm{mmol})$ at room temperature. After stirring for $30 \mathrm{~min}$, the solvent and HMDS were removed in vacuo to give an oily product. The crude product was extracted by pentane $(1.5 \mathrm{~mL})$ for recrystallization. The colorless single crystals of 2 were obtained at $-20{ }^{\circ} \mathrm{C}$ overnight (10.2 mg, 66 \%). Method B: $\mathrm{IPr}(4.6 \mathrm{mg}, 0.0305 \mathrm{mmol})$ was added into a $\mathrm{C}_{6} \mathrm{H}_{6}(2 \mathrm{~mL})$ solution of 1 (20 mg, $0.0305 \mathrm{mmol}$ ) at room temperature. After stirring for $30 \mathrm{~min}$, the solvent was removed in vacuo to give an oily crude product, which was extracted by pentane $(1.5 \mathrm{~mL})$. Storing the pentane solution at $-20{ }^{\circ} \mathrm{C}$ overnight gave 2 as colorless single crystals $(11.0 \mathrm{mg}$, $71 \%$ ). ${ }^{1} \mathrm{H}\left\{{ }^{31} \mathrm{P}\right\}$ NMR (400 MHz, $\left.\mathrm{CDCl}_{3}\right): \delta$ (ppm) 6.07 (dd (pseudo triplet), ${ }^{3} J_{\mathrm{H}-\mathrm{H}} \approx{ }^{3} J_{\mathrm{H}-\mathrm{H}}=8$ $\mathrm{Hz}, 1 \mathrm{H}$, Alkenyl- $H), 5.86\left(\mathrm{~d},{ }^{3} \mathrm{~J}_{\mathrm{H}-\mathrm{H}}=8 \mathrm{~Hz}, 1 \mathrm{H}\right.$, Alkenyl- $\left.H\right), 3.59\left(\mathrm{~m}, 1 \mathrm{H}, \mathrm{PCH}_{2} \mathrm{CH}\right), 2.85$ 
(sept, ${ }^{3} J_{\mathrm{H}-\mathrm{H}}=7 \mathrm{~Hz}, 1 \mathrm{H}, \mathrm{C} / \mathrm{MMe}_{2}$ ), $2.28\left(\mathrm{sept},{ }^{3} \mathrm{H}_{\mathrm{H}-\mathrm{H}}=7 \mathrm{~Hz}, 1 \mathrm{H}, \mathrm{C} / \mathrm{Me}_{2}\right), 2.02-1.91(\mathrm{~m}$, $\sim 13 \mathrm{H}, \mathrm{Ad}-\mathrm{H}$ and/or $\left.\mathrm{CH}_{2}\right), 1.72-1.50\left(\mathrm{~m}, \sim 10 \mathrm{H}, \mathrm{Ad}-\mathrm{H}\right.$ and/or $\left.\mathrm{CH}_{2}\right), 1.34(\mathrm{~s}, 3 \mathrm{H}, \mathrm{CMe})$, $1.32(\mathrm{~s}, 3 \mathrm{H}, \mathrm{CMe}), 1.23\left(\mathrm{~d},{ }^{3} \mathrm{~J}_{\mathrm{H}-\mathrm{H}}=7 \mathrm{~Hz}, 3 \mathrm{H}, \mathrm{CH} M e_{2}\right), 1.02\left(\mathrm{~d},{ }^{3} J_{\mathrm{H}-\mathrm{H}}=7 \mathrm{~Hz}, 3 \mathrm{H}\right.$, $\left.\mathrm{CH} M e_{2}\right), 0.988\left(\mathrm{~d},{ }^{3} \mathrm{JH}_{\mathrm{H}-\mathrm{H}}=7 \mathrm{~Hz}, 3 \mathrm{H}, \mathrm{CH} M e_{2}\right), 0.987\left(\mathrm{t},{ }^{3} \mathcal{H}_{\mathrm{H}-\mathrm{H}}=7 \mathrm{~Hz}, 3 \mathrm{H}, \mathrm{CH}_{2} M e\right), 0.89(\mathrm{t}$, $\left.{ }^{3} J_{\mathrm{H}-\mathrm{H}}=7 \mathrm{~Hz}, 3 \mathrm{H}, \mathrm{CH}_{2} M e\right), 0.88\left(\mathrm{~d},{ }^{3} J_{\mathrm{H}-\mathrm{H}}=7 \mathrm{~Hz}, 3 \mathrm{H}, \mathrm{CH} M e_{2}\right) .{ }^{1} \mathrm{H}$ NMR $(400 \mathrm{MHz}$, $\mathrm{CDCl}_{3}$ ): $\delta(\mathrm{ppm}) 6.07$ (ddd, ${ }^{3} \mathrm{~J}_{\mathrm{H}-\mathrm{H}}=8 \mathrm{~Hz},{ }^{3} J_{\mathrm{H}-\mathrm{H}}=7 \mathrm{~Hz},{ }^{4} J_{\mathrm{P}-\mathrm{H}}=4 \mathrm{~Hz}, 1 \mathrm{H}$, Alkenyl- $H$ ), 5.86 $\left(\mathrm{ddd},{ }^{3} J_{\mathrm{H}-\mathrm{H}}=8 \mathrm{~Hz},{ }^{4} J_{\mathrm{H}-\mathrm{H}}=2 \mathrm{~Hz},{ }^{3} \mathrm{~J}_{\mathrm{P}-\mathrm{H}}=2 \mathrm{~Hz}, 1 \mathrm{H}\right.$, Alkenyl- $H$ ), $3.59\left(\mathrm{~m}, 1 \mathrm{H}, \mathrm{PCH}_{2} \mathrm{CH}\right)$, 2.85 (sept, ${ }^{3} \mathrm{~J}_{\mathrm{H}-\mathrm{H}}=7 \mathrm{~Hz}, 1 \mathrm{H}, \mathrm{C} / \mathrm{MMe}_{2}$ ), 2.28 (sept, ${ }^{3} \mathrm{~J}_{\mathrm{H}-\mathrm{H}}=7 \mathrm{~Hz}, 1 \mathrm{H}, \mathrm{C} / \mathrm{Me}_{2}$ ), 2.02-1.91 (m, $\sim 13 \mathrm{H}, \mathrm{Ad}-H$ and/or $\left.\mathrm{CH}_{2}\right), 1.72-1.50\left(\mathrm{~m}, \sim 10 \mathrm{H}, \mathrm{Ad}-H\right.$ and/or $\left.\mathrm{CH}_{2}\right), 1.34(\mathrm{~s}, 3 \mathrm{H}$, $\mathrm{CMe}_{2}$ ), $1.32\left(\mathrm{~s}, 3 \mathrm{H}, \mathrm{C} M e_{2}\right), 1.23\left(\mathrm{dd},{ }^{3} \mathrm{~J}_{\mathrm{H}-\mathrm{H}}=7 \mathrm{~Hz},{ }^{4} \mathrm{JP}_{-\mathrm{H}}=3 \mathrm{~Hz}, 3 \mathrm{H}, \mathrm{CH} M e_{2}\right), 1.02(\mathrm{~d}$, $\left.{ }^{3} J_{\mathrm{H}-\mathrm{H}}=7 \mathrm{~Hz}, 3 \mathrm{H}, \mathrm{CH} M e_{2}\right), 0.988\left(\mathrm{~d},{ }^{3} \mathrm{~J}_{\mathrm{H}-\mathrm{H}}=7 \mathrm{~Hz}, 3 \mathrm{H}, \mathrm{CH} M e_{2}\right), 0.987\left(\mathrm{t},{ }^{3} \int_{\mathrm{H}-\mathrm{H}}=7 \mathrm{~Hz}\right.$, $\left.3 \mathrm{H}, \mathrm{CH}_{2} M e\right), 0.89\left(\mathrm{t},{ }^{3} \mathrm{~J}_{\mathrm{H}-\mathrm{H}}=7 \mathrm{~Hz}, 3 \mathrm{H}, \mathrm{CH}_{2} M e\right), 0.88\left(\mathrm{~d},{ }^{3} \mathrm{~J}_{\mathrm{H}-\mathrm{H}}=7 \mathrm{~Hz}, 3 \mathrm{H}, \mathrm{CH} M e_{2}\right)$. ${ }^{13} \mathrm{C}\left\{{ }^{1} \mathrm{H}\right\} \operatorname{NMR}\left(126 \mathrm{MHz}, \mathrm{CDCl}_{3}\right): \delta(\mathrm{ppm}) 159.6\left(\mathrm{~d}, J_{\mathrm{C}-\mathrm{P}}=6 \mathrm{~Hz}\right), 147.4\left(\mathrm{~d}, J_{\mathrm{C}-\mathrm{P}}=5 \mathrm{~Hz}\right), 136.2$ $\left(\mathrm{d}, J_{\mathrm{C}-\mathrm{P}}=2 \mathrm{~Hz}\right), 132.3\left(\mathrm{~d}, J_{\mathrm{C}-\mathrm{P}}=6 \mathrm{~Hz}\right), 127.7\left(\mathrm{~d}, J_{\mathrm{C}-\mathrm{P}}=3 \mathrm{~Hz}\right), 104.7\left(\mathrm{~d}, J_{\mathrm{C}-\mathrm{P}}=39 \mathrm{~Hz}\right)($ AlkenylC), $59.9(\mathrm{~s}), 52.7(\mathrm{~s}), 50.9\left(\mathrm{~d}, J_{\mathrm{C}-\mathrm{P}}=17 \mathrm{~Hz}\right), 49.1(\mathrm{~s}), 45.6\left(\mathrm{~d}, J_{\mathrm{C}-\mathrm{P}}=41 \mathrm{~Hz}\right), 44.6\left(\mathrm{~d}, J_{\mathrm{C}-\mathrm{P}}=15\right.$ $\mathrm{Hz}), 39.8\left(\mathrm{~d}, J_{\mathrm{C}-\mathrm{P}}=27 \mathrm{~Hz}\right), 37.0\left(\mathrm{~d}, J_{\mathrm{C}-\mathrm{P}}=1 \mathrm{~Hz}\right), 34.7\left(\mathrm{~d}, J_{\mathrm{C}-\mathrm{P}}=5 \mathrm{~Hz}\right), 32.0(\mathrm{~s}), 31.9(\mathrm{~s}), 30.9(\mathrm{~d}$, $\left.J_{\mathrm{C}-\mathrm{P}}=16 \mathrm{~Hz}\right), 29.7(\mathrm{~s}), 29.1(\mathrm{~s}), 25.6\left(\mathrm{~d}, J_{\mathrm{C}-\mathrm{P}}=13 \mathrm{~Hz}\right), 24.9\left(\mathrm{~d}, J_{\mathrm{C}-\mathrm{P}}=4 \mathrm{~Hz}\right), 23.4(\mathrm{~s}), 21.8\left(\mathrm{~d}, J_{\mathrm{C}-}\right.$ $\mathrm{P}=27 \mathrm{~Hz}), 21.0(\mathrm{~s}), 9.9\left(\mathrm{~d}, J_{\mathrm{C}-\mathrm{P}}=36 \mathrm{~Hz}\right)($ Saturated $C), 34.3,21.7,14.2($ pentane- $C) .{ }^{31} \mathrm{P}$ NMR $\left(162 \mathrm{MHz}, \mathrm{CDCl}_{3}\right): \delta(\mathrm{ppm}) 3.9\left(\mathrm{dm}, \mathrm{JP}_{\mathrm{H}}=25 \mathrm{~Hz}\right) .{ }^{31} \mathrm{P}\left\{{ }^{1} \mathrm{H}\right\} \mathrm{NMR}\left(162 \mathrm{MHz}, \mathrm{CDCl}_{3}\right): \delta(\mathrm{ppm})$ 3.9 (s). MS (DART) $[\mathrm{M}+1] \mathrm{C}_{34} \mathrm{H}_{53} \mathrm{NP}^{+}$calc. $506.3910 \mathrm{~m} / \mathrm{z}$, found $506.3908 \mathrm{~m} / \mathrm{z}$. 
Synthesis and low temperature isolation of 4 . $\mathrm{IPr}(4 \mathrm{mg}, 0.0260 \mathrm{mmol})$ was added into a $\mathrm{FC}_{6} \mathrm{H}_{5}$ $(2 \mathrm{~mL})$ solution of $3(15 \mathrm{mg}, 0.0260 \mathrm{mmol})$ at $-20{ }^{\circ} \mathrm{C}$. After stirring for $20 \mathrm{~min}$ at $-20{ }^{\circ} \mathrm{C}$, the solvent was removed at $-20{ }^{\circ} \mathrm{C}$ in vacuo to give an oily crude product, which was extracted by cold pentane $(1.5 \mathrm{~mL})$. After removing pentane, 4 was isolated as slightly yellow oil (3.8 $\mathrm{mg}$, $34 \%) .{ }^{1} \mathrm{H}\left\{{ }^{31} \mathrm{P}\right\} \operatorname{NMR}\left(500 \mathrm{MHz}, \mathrm{CDCl}_{3},-20{ }^{\circ} \mathrm{C}\right): \delta(\mathrm{ppm}) 7.18\left(\mathrm{t},{ }^{3} \mathrm{~J}_{\mathrm{H}-\mathrm{H}}=7 \mathrm{~Hz}, 1 \mathrm{H}, \mathrm{Ar} H\right)$, $7.12\left(\mathrm{~d},{ }^{3} J_{\mathrm{H}-\mathrm{H}}=7 \mathrm{~Hz}, 2 \mathrm{H}, \mathrm{ArH}\right), 6.92\left(\mathrm{~d},{ }^{2} \mathcal{J}_{\mathrm{H}-\mathrm{H}}=5 \mathrm{~Hz}, 1 \mathrm{H}, \mathrm{P}=\mathrm{C} H_{2}\right), 6.88\left(\mathrm{~d},{ }^{2}{ } \mathrm{H}_{\mathrm{H}}=5 \mathrm{~Hz}\right.$, 1H, $\left.\mathrm{P}=\mathrm{C} H_{2}\right), 3.49\left(\mathrm{~m}, 2 \mathrm{H}, \mathrm{C} H \mathrm{Me}_{2}\right), 2.40\left(\mathrm{~m}, 2 \mathrm{H}, \mathrm{CH}_{2} \mathrm{Me}\right), 2.09(\mathrm{~m}, 2 \mathrm{H}, \mathrm{CH} / \mathrm{Me}), 1.88(\mathrm{~s}$, $\left.2 \mathrm{H}, \mathrm{CH}_{2}\right), 1.26\left(\mathrm{~d},{ }^{3} \mathrm{~J}_{\mathrm{H}-\mathrm{H}}=6 \mathrm{~Hz}, 6 \mathrm{H}, \mathrm{CH} M e_{2}\right), 1.23\left(\mathrm{~s}, 6 \mathrm{H}, \mathrm{C} M e_{2}\right), 1.21\left(\mathrm{~d},{ }^{3} J_{\mathrm{H}-\mathrm{H}}=6 \mathrm{~Hz}\right.$, $\left.6 \mathrm{H}, \mathrm{CH} M e_{2}\right), 1.02\left(\mathrm{t},{ }^{3} \mathrm{~J}_{\mathrm{H}-\mathrm{H}}=7 \mathrm{~Hz}, 3 \mathrm{H}, \mathrm{CH}_{2} M e\right), 0.87\left(\mathrm{t},{ }^{3} J_{\mathrm{H}-\mathrm{H}}=7 \mathrm{~Hz}, 3 \mathrm{H}, \mathrm{CH}_{2} M e\right), 0.81$ (s, 9H, CMe $).{ }^{1} \mathrm{H}$ NMR $\left(500 \mathrm{MHz}, \mathrm{CDCl}_{3},-20^{\circ} \mathrm{C}\right): \delta(\mathrm{ppm}) 7.18\left(\mathrm{t},{ }^{3} J_{\mathrm{H}-\mathrm{H}}=7 \mathrm{~Hz}, 1 \mathrm{H}\right.$, $\operatorname{Ar} H), 7.12\left(\mathrm{~d},{ }^{3} J_{\mathrm{H}-\mathrm{H}}=7 \mathrm{~Hz}, 2 \mathrm{H}, \mathrm{Ar} H\right), 6.92\left(\mathrm{dd},{ }^{2} J_{\mathrm{P}-\mathrm{H}}=28 \mathrm{~Hz},{ }^{2} \mathcal{J}_{\mathrm{H}-\mathrm{H}}=5 \mathrm{~Hz}, 1 \mathrm{H}, \mathrm{P}=\mathrm{C} H_{2}\right)$, $6.88\left(\mathrm{dd},{ }^{2} \mathrm{JP}_{-\mathrm{H}}=33 \mathrm{~Hz},{ }^{2} \mathrm{~J}_{\mathrm{H}-\mathrm{H}}=5 \mathrm{~Hz}, 1 \mathrm{H}, \mathrm{P}=\mathrm{C} H_{2}\right), 3.49\left(\mathrm{~m}, 2 \mathrm{H}, \mathrm{C} / \mathrm{Me}_{2}\right), 2.40(\mathrm{~m}, 2 \mathrm{H}$, $\left.\mathrm{CH}_{2} \mathrm{Me}\right), 2.09\left(\mathrm{~m}, 2 \mathrm{H}, \mathrm{CH} H_{2} \mathrm{Me}\right), 1.88\left(\mathrm{~s}, 2 \mathrm{H}, \mathrm{CH}_{2}\right), 1.26\left(\mathrm{~d},{ }^{3} \mathrm{~J}_{\mathrm{H}-\mathrm{H}}=6 \mathrm{~Hz}, 6 \mathrm{H}, \mathrm{CH} M e_{2}\right)$, $1.23(\mathrm{~s}, 6 \mathrm{H}, \mathrm{CMe}), 1.21\left(\mathrm{~d},{ }^{3} \mathrm{~J}_{\mathrm{H}-\mathrm{H}}=6 \mathrm{~Hz}, 6 \mathrm{H}, \mathrm{CH} M e_{2}\right), 1.02\left(\mathrm{t},{ }^{3} J_{\mathrm{H}-\mathrm{H}}=7 \mathrm{~Hz}, 3 \mathrm{H}, \mathrm{CH}_{2} M e\right)$, $0.87\left(\mathrm{t},{ }^{3} \mathrm{~J}_{\mathrm{H}-\mathrm{H}}=7 \mathrm{~Hz}, 3 \mathrm{H}, \mathrm{CH}_{2} \mathrm{Me}\right), 0.81(\mathrm{~s}, 9 \mathrm{H}, \mathrm{CMe}) .{ }^{31} \mathrm{P} \mathrm{NMR}\left(162 \mathrm{MHz}, \mathrm{C}_{6} \mathrm{D}_{6}, 6{ }^{\circ} \mathrm{C}\right): \delta$ (ppm) $303.1\left(t,{ }^{2} \mathrm{JP}_{\mathrm{PH}}=31 \mathrm{~Hz}\right) \cdot{ }^{31} \mathrm{P}\left\{{ }^{1} \mathrm{H}\right\} \mathrm{NMR}\left(162 \mathrm{MHz}, \mathrm{C}_{6} \mathrm{D}_{6}, 6{ }^{\circ} \mathrm{C}\right): \delta(\mathrm{ppm}) 303.1(\mathrm{~s}) . \mathrm{MS}$ (DART) $[\mathrm{M}+1] \mathrm{C}_{28} \mathrm{H}_{47} \mathrm{NP}^{+}$calc. $428.34461 \mathrm{~m} / \mathrm{z}$, found $428.34472 \mathrm{~m} / \mathrm{z}$.

Synthesis of 5. Method A: $\mathrm{C}_{6} \mathrm{H}_{6}(2 \mathrm{~mL})$ was added into a mixture of KHMDS (5.2 mg, 0.0260 $\mathrm{mmol})$ and $3(15 \mathrm{mg}, 0.0260 \mathrm{mmol})$ at room temperature. After stirring for $6 \mathrm{~h}$, the solvent and HMDS were removed in vacuo to give an oily product. The crude product was extracted by pentane $(1.5 \mathrm{~mL})$ for recrystallization. The colorless single crystals of 5 were obtained at $-20{ }^{\circ} \mathrm{C}$ after 2 days ( $7.6 \mathrm{mg}, 68 \%$ ). Method B: $\operatorname{IPr}(4 \mathrm{mg}, 0.0260 \mathrm{mmol})$ was added into a $\mathrm{C}_{6} \mathrm{H}_{6}(2 \mathrm{~mL})$ solution of $3(15 \mathrm{mg}, 0.0260 \mathrm{mmol})$ at room temperature. After stirring for $6 \mathrm{~h}$, the solvent was removed in vacuo to give an oily crude product, which was extracted by pentane $(1.5 \mathrm{~mL})$. 
Storing the pentane solution at $-20{ }^{\circ} \mathrm{C}$ for 2 days gave 5 as colorless single crystals $(7.8 \mathrm{mg}$, $70 \%$ \%. ${ }^{1} \mathrm{H}\left\{{ }^{31} \mathrm{P}\right\}$ NMR (400 MHz, $\mathrm{C}_{6} \mathrm{D}_{6}$ ): $\delta$ (ppm) 6.03 (dd (pseudo triplet), ${ }^{3} J_{\mathrm{H}-\mathrm{H}} \approx{ }^{3} J_{\mathrm{H}-\mathrm{H}}=7$ $\mathrm{Hz}, 1 \mathrm{H}$, Alkenyl- $H$ ), $5.93\left(\mathrm{dd},{ }^{3} \mathrm{~J}_{\mathrm{H}-\mathrm{H}}=8 \mathrm{~Hz},{ }^{4} \mathrm{~J}_{\mathrm{H}-\mathrm{H}}=2 \mathrm{~Hz}, 1 \mathrm{H}\right.$, Alkenyl- $\left.H\right), 3.50(\mathrm{~m}, 1 \mathrm{H}$, $\mathrm{PCH}_{2} \mathrm{CH}$ ), 2.91 (sept, ${ }^{3} \mathrm{~J}_{\mathrm{H}-\mathrm{H}}=7 \mathrm{~Hz}, 1 \mathrm{H}, \mathrm{C} / \mathrm{Me}_{2}$ ), 2.42 (sept, ${ }^{3} \mathrm{~J}_{\mathrm{H}-\mathrm{H}}=7 \mathrm{~Hz}, 1 \mathrm{H}, \mathrm{C} / \mathrm{Me}_{2}$ ), $2.10\left(\mathrm{dd},{ }^{2} J_{\mathrm{H}-\mathrm{H}}=12 \mathrm{~Hz},{ }^{3} J_{\mathrm{H}-\mathrm{H}}=2 \mathrm{~Hz}, 1 \mathrm{H}, \mathrm{PC} H_{2}\right), 1.99 \sim 1.77\left(\mathrm{~m}, 4 \mathrm{H}, \mathrm{CH}_{2}\right), 1.66 \sim 1.51(\mathrm{~m}$, $\left.3 \mathrm{H}, \mathrm{CH}_{2}\right), 1.46\left(\mathrm{~d},{ }^{3} \mathrm{~J}_{\mathrm{H}-\mathrm{H}}=7 \mathrm{~Hz}, 3 \mathrm{H}, \mathrm{CH} M e_{2}\right), 1.44(\mathrm{~s}, 9 \mathrm{H}, \mathrm{CMe}), 1.28(\mathrm{~s}, 3 \mathrm{H}, \mathrm{CMe}$ ), $1.27(\mathrm{~s}, 3 \mathrm{H}, \mathrm{CMe}), 1.08\left(\mathrm{~d},{ }^{3} \mathrm{~J}_{\mathrm{H}-\mathrm{H}}=7 \mathrm{~Hz}, 3 \mathrm{H}, \mathrm{CH} M e_{2}\right), 1.06\left(\mathrm{~d},{ }^{3} J_{\mathrm{H}-\mathrm{H}}=7 \mathrm{~Hz}, 3 \mathrm{H}\right.$, $\left.\mathrm{CH} M e_{2}\right), 0.93\left(\mathrm{t},{ }^{3} J_{\mathrm{H}-\mathrm{H}}=7 \mathrm{~Hz}, 3 \mathrm{H}, \mathrm{CH}_{2} M e\right), 0.91\left(\mathrm{~d},{ }^{3} J_{\mathrm{H}-\mathrm{H}}=7 \mathrm{~Hz}, 3 \mathrm{H}, \mathrm{CH} M e_{2}\right), 0.85(\mathrm{t}$, $\left.{ }^{3} J_{\mathrm{H}-\mathrm{H}}=7 \mathrm{~Hz}, 3 \mathrm{H}, \mathrm{CH}_{2} M e\right) .{ }^{1} \mathrm{H}$ NMR $\left(400 \mathrm{MHz}, \mathrm{C}_{6} \mathrm{D}_{6}\right): \delta(\mathrm{ppm}) 6.03\left(\mathrm{ddd},{ }^{3} \mathrm{H}_{\mathrm{H}-\mathrm{H}}=8 \mathrm{~Hz}\right.$, ${ }^{3} J_{\mathrm{H}-\mathrm{H}}=7 \mathrm{~Hz},{ }^{3} \mathrm{~J}_{\mathrm{H}-\mathrm{P}}=4 \mathrm{~Hz}, 1 \mathrm{H}$, Alkenyl- $H$ ), 5.93 (ddd (pseudo dt), ${ }^{3} \mathrm{~J}_{\mathrm{H}-\mathrm{H}}=8 \mathrm{~Hz},{ }^{4} \mathrm{~J}_{\mathrm{H}-\mathrm{H}} \approx$ ${ }^{3} \mathrm{P}_{\mathrm{P}-\mathrm{H}}=2 \mathrm{~Hz}, 1 \mathrm{H}$, Alkenyl- $H$ ) $, 3.50\left(\mathrm{~m}, 1 \mathrm{H}, \mathrm{PCH}_{2} \mathrm{CH}\right), 2.91$ (sept, ${ }^{3} \mathrm{~J}_{\mathrm{H}-\mathrm{H}}=7 \mathrm{~Hz}, 1 \mathrm{H}$, $\mathrm{C} / \mathrm{Me}_{2}$ ), $2.42\left(\mathrm{sept},{ }^{3} \mathrm{JH}_{\mathrm{H}-\mathrm{H}}=7 \mathrm{~Hz}, 1 \mathrm{H}, \mathrm{C} / \mathrm{Me}_{2}\right.$ ), 2.10 (ddd, ${ }^{2} J_{\mathrm{H}-\mathrm{P}}=27 \mathrm{~Hz},{ }^{2} J_{\mathrm{H}-\mathrm{H}}=12 \mathrm{~Hz}$, $\left.{ }^{3} J_{\mathrm{H}-\mathrm{H}}=2 \mathrm{~Hz}, 1 \mathrm{H}, \mathrm{PC} H_{2}\right), 1.99 \sim 1.77\left(\mathrm{~m}, 4 \mathrm{H}, \mathrm{CH} \mathrm{H}_{2}\right), 1.66 \sim 1.51\left(\mathrm{~m}, 3 \mathrm{H}, \mathrm{CH}_{2}\right), 1.47\left(\mathrm{dd},{ }^{3} \mathrm{H}_{\mathrm{H}-}\right.$ $\left.\mathrm{H}=7 \mathrm{~Hz},{ }^{4} J_{\mathrm{H}-\mathrm{P}}=3 \mathrm{~Hz}, 3 \mathrm{H}, \mathrm{CH} M e_{2}\right), 1.44(\mathrm{~s}, 9 \mathrm{H}, \mathrm{CMe}), 1.28(\mathrm{~s}, 3 \mathrm{H}, \mathrm{CMe}), 1.27(\mathrm{~s}, 3 \mathrm{H}$, $\mathrm{CMe}$ ), $1.08\left(\mathrm{~d},{ }^{3} \mathcal{J}_{\mathrm{H}-\mathrm{H}}=7 \mathrm{~Hz}, 3 \mathrm{H}, \mathrm{CH} M e_{2}\right), 1.06\left(\mathrm{~d},{ }^{3} J_{\mathrm{H}-\mathrm{H}}=7 \mathrm{~Hz}, 3 \mathrm{H}, \mathrm{CH} M e_{2}\right), 0.93(\mathrm{t}$, $\left.{ }^{3} J_{\mathrm{H}-\mathrm{H}}=7 \mathrm{~Hz}, 3 \mathrm{H}, \mathrm{CH}_{2} M e\right), 0.91\left(\mathrm{~d},{ }^{3} J_{\mathrm{H}-\mathrm{H}}=7 \mathrm{~Hz}, 3 \mathrm{H}, \mathrm{CH} M e_{2}\right), 0.85\left(\mathrm{t},{ }^{3} J_{\mathrm{H}-\mathrm{H}}=7 \mathrm{~Hz}, 3 \mathrm{H}\right.$, $\left.\mathrm{CH}_{2} M e\right) \cdot{ }^{13} \mathrm{C}\left\{{ }^{1} \mathrm{H}\right\} \operatorname{NMR}\left(126 \mathrm{MHz}, \mathrm{C}_{6} \mathrm{D}_{6}\right): 159.1\left(\mathrm{~d}, J_{\mathrm{C}-\mathrm{P}}=5 \mathrm{~Hz}\right), 147.5\left(\mathrm{~d}, J_{\mathrm{C}-\mathrm{P}}=5 \mathrm{~Hz}\right), 136.6$ $\left(\mathrm{d}, J_{\mathrm{C}-\mathrm{P}}=1 \mathrm{~Hz}\right), 132.3\left(\mathrm{~d}, J_{\mathrm{C}-\mathrm{P}}=6 \mathrm{~Hz}\right), 103.6\left(\mathrm{~d}, J_{\mathrm{C}-\mathrm{P}}=43 \mathrm{~Hz}\right)($ Alkenyl- $C), 137.9,129.3,128.6$, 125.7, 21.4 (toluene-C), $59.8(\mathrm{~s}), 52.3(\mathrm{~s}), 51.9\left(\mathrm{~d}, J_{\mathrm{C}-\mathrm{P}}=18 \mathrm{~Hz}\right), 49.0(\mathrm{~s}), 45.4\left(\mathrm{~d}, J_{\mathrm{C}-\mathrm{P}}=45 \mathrm{~Hz}\right)$, $37.4\left(\mathrm{~d}, J_{\mathrm{C}-\mathrm{P}}=30 \mathrm{~Hz}\right), 35.1\left(\mathrm{~d}, J_{\mathrm{C}-\mathrm{P}}=5 \mathrm{~Hz}\right), 34.7\left(\mathrm{~d}, J_{\mathrm{C}-\mathrm{P}}=14 \mathrm{~Hz}\right), 32.3(\mathrm{~s}), 31.9(\mathrm{~s}), 30.7\left(\mathrm{~d}, J_{\mathrm{C}-}\right.$ $\mathrm{P}=2 \mathrm{~Hz}), 29.3(\mathrm{~s}), 26.0\left(\mathrm{~d}, J_{\mathrm{C}-\mathrm{P}}=13 \mathrm{~Hz}\right), 25.1\left(\mathrm{~d}, J_{\mathrm{C}-\mathrm{P}}=4 \mathrm{~Hz}\right), 23.5(\mathrm{~s}), 22.0\left(\mathrm{~d}, J_{\mathrm{C}-\mathrm{P}}=28 \mathrm{~Hz}\right)$, 
$20.8(\mathrm{~s}), 9.9\left(\mathrm{~d}, J_{\mathrm{C}-\mathrm{P}}=39 \mathrm{~Hz}\right)($ Saturated $C), 34.6,22.7,14.3$ (pentane-C). ${ }^{31} \mathrm{P}$ NMR $(162 \mathrm{MHz}$ $\left.\mathrm{C}_{6} \mathrm{D}_{6}\right): \delta(\mathrm{ppm}) 7.5\left(\mathrm{dm}, \mathrm{JP}_{\mathrm{H}}=27 \mathrm{~Hz}\right) \cdot{ }^{31} \mathrm{P}\left\{{ }^{1} \mathrm{H}\right\} \mathrm{NMR}\left(162 \mathrm{MHz}, \mathrm{C}_{6} \mathrm{D}_{6}\right): \delta(\mathrm{ppm}) 7.5(\mathrm{~s}) . \mathrm{MS}$ (DART) $[\mathrm{M}+1] \mathrm{C}_{28} \mathrm{H}_{47} \mathrm{NP}^{+}$calc. $428.34461 \mathrm{~m} / \mathrm{z}$, found $428.34467 \mathrm{~m} / \mathrm{z}$.

Synthesis of 6. $\mathrm{C}_{6} \mathrm{H}_{6}(2 \mathrm{~mL})$ was added into a mixture of freshly generated $2(9.3 \mathrm{mg}, 0.0183$ $\mathrm{mmol})$ and $\mathrm{S}_{8}(0.6 \mathrm{mg}, 0.0023 \mathrm{mmol})$. After stirring for $30 \mathrm{~min}$, the solvent was removed in vacuo to give 6 as a white solid $(9.7 \mathrm{mg}, 99 \%)$. Colorless single crystals were grown in a saturated pentane solution of 6 at $-20{ }^{\circ} \mathrm{C}$ overnight. ${ }^{1} \mathrm{H}\left\{{ }^{31} \mathrm{P}\right\}$ NMR $\left(400 \mathrm{MHz}, \mathrm{CDCl}_{3}\right): \delta(\mathrm{ppm})$ 6.30 (dd (pseudo triplet), ${ }^{3} J_{\mathrm{H}-\mathrm{H}} \approx{ }^{3} J_{\mathrm{H}-\mathrm{H}}=8 \mathrm{~Hz}, 1 \mathrm{H}$, Alkenyl- $\left.H\right), 6.19\left(\mathrm{~d},{ }^{3} J_{\mathrm{H}-\mathrm{H}}=8 \mathrm{~Hz}, 1 \mathrm{H}\right.$, Alkenyl- $H$ ), $3.55\left(\mathrm{~m}, 1 \mathrm{H}, \mathrm{PCH}_{2} \mathrm{CH}\right), 3.06\left(\mathrm{sept},{ }^{3} J_{\mathrm{H}-\mathrm{H}}=7 \mathrm{~Hz}, 1 \mathrm{H}, \mathrm{C}-\mathrm{Me}_{2}\right), 2.71$ (dd, ${ }^{2} J_{\mathrm{H}-\mathrm{H}}$ $\left.=13 \mathrm{~Hz},{ }^{3} \mathrm{~J}_{\mathrm{H}-\mathrm{H}}=4 \mathrm{~Hz}, 1 \mathrm{H}, \mathrm{PC} H_{2}\right), 2.64-2.28\left(\mathrm{~m}, \sim 7 \mathrm{H}, \mathrm{C} / \mathrm{Me}_{2}\right.$ and/or Ad- $H$ and/or $\left.\mathrm{CH}_{2}\right)$, 2.22-1.84 (m, 8H, CHMe 2 and/or Ad- $H$ and/or $\left.\mathrm{CH}_{2}\right), 1.73\left(\mathrm{~m}, 4 \mathrm{H}, \mathrm{Ad}-\mathrm{H}\right.$ and/or $\left.\mathrm{CH}_{2}\right)$, $1.64\left(\mathrm{~d},{ }^{3} \mathrm{~J}_{\mathrm{H}-\mathrm{H}}=7 \mathrm{~Hz}, 3 \mathrm{H}, \mathrm{CH} M e_{2}\right), 1.58(\mathrm{~m}, 3 \mathrm{H}, \mathrm{Ad}-H), 1.44\left(\mathrm{~s}, 3 \mathrm{H}, \mathrm{C} M e_{2}\right), 1.29(\mathrm{~s}, 3 \mathrm{H}$, $\mathrm{CMe}_{2}$, overlapped with pentane- $\left.\mathrm{CH}_{2}\right), 1.08\left(\mathrm{t},{ }^{3} \mathrm{~J}_{\mathrm{H}-\mathrm{H}}=7 \mathrm{~Hz}, 3 \mathrm{H}, \mathrm{CH}_{2} \mathrm{Me}\right), 1.04-0.93(\mathrm{~m}$, $12 \mathrm{H}, \mathrm{CHMe}$ and $\left.\mathrm{CH}_{2} M e\right), 0.88\left(\mathrm{t},{ }^{3} \mathrm{~J}_{\mathrm{H}-\mathrm{H}}=7 \mathrm{~Hz}\right.$, pentane-Me). ${ }^{1} \mathrm{H}$ NMR $(400 \mathrm{MHz}$, $\mathrm{CDCl}_{3}$ ): $\delta(\mathrm{ppm}) 6.30$ (ddd (pseudo quartet), ${ }^{3} \mathrm{~J}_{\mathrm{H}-\mathrm{H}} \approx{ }^{3} J_{\mathrm{H}-\mathrm{H}} \approx{ }^{4} J_{\mathrm{P}-\mathrm{H}}=7 \mathrm{~Hz}, 1 \mathrm{H}$, Alkenyl- $H$ ), $6.19\left(\mathrm{dd},{ }^{3} \mathrm{~J}_{\mathrm{H}-\mathrm{H}}=8 \mathrm{~Hz},{ }^{3} \mathrm{JP}_{\mathrm{P}-\mathrm{H}}=3 \mathrm{~Hz}, 1 \mathrm{H}\right.$, Alkenyl- $\left.H\right), 3.55\left(\mathrm{dm},{ }^{3} \mathrm{JP}_{\mathrm{P}-\mathrm{H}}=22 \mathrm{~Hz}, 1 \mathrm{H}\right.$, $\mathrm{PCH}_{2} \mathrm{CH}$ ), 3.06 (sept, ${ }^{3} \mathrm{~J}_{\mathrm{H}-\mathrm{H}}=7 \mathrm{~Hz}, 1 \mathrm{H}, \mathrm{C} / \mathrm{MMe}_{2}$ ), 2.71 (ddd (pseudo dt), ${ }^{2} \mathrm{JH}_{\mathrm{H}-\mathrm{H}}=13 \mathrm{~Hz}$, $\left.{ }^{3} J_{\mathrm{H}-\mathrm{H}} \approx{ }^{2} J_{\mathrm{P}-\mathrm{H}}=4 \mathrm{~Hz}, 1 \mathrm{H}, \mathrm{PC} H_{2}\right), 2.64-2.28\left(\mathrm{~m}, \sim 7 \mathrm{H}, \mathrm{C} / \mathrm{Mee}_{2}\right.$ and/or $\mathrm{Ad}-\mathrm{H}$ and/or $\left.\mathrm{CH}_{2}\right)$, 2.22-1.84 (m, 8H, CHMe 2 and/or Ad- $H$ and/or $\left.\mathrm{CH}_{2}\right), 1.73\left(\mathrm{~m}, 4 \mathrm{H}, \mathrm{Ad}-\mathrm{H}\right.$ and/or $\left.\mathrm{CH}_{2}\right)$, $1.64\left(\mathrm{~d},{ }^{3} J_{\mathrm{H}-\mathrm{H}}=7 \mathrm{~Hz}, 3 \mathrm{H}, \mathrm{CH} M e_{2}\right), 1.58(\mathrm{~m}, 3 \mathrm{H}, \mathrm{Ad}-H), 1.44\left(\mathrm{~s}, 3 \mathrm{H}, \mathrm{CM} e_{2}\right), 1.29(\mathrm{~s}, 3 \mathrm{H}$, $\mathrm{CMe}_{2}$, overlapped with pentane- $\left.\mathrm{CH}_{2}\right), 1.08\left(\mathrm{t},{ }^{3} \mathrm{~J}_{\mathrm{H}-\mathrm{H}}=7 \mathrm{~Hz}, 3 \mathrm{H}, \mathrm{CH}_{2} \mathrm{Me}\right), 1.04-0.93(\mathrm{~m}$, 
$12 \mathrm{H}, \mathrm{CHMe} e_{2}$ and $\left.\mathrm{CH}_{2} M e\right), 0.88\left(\mathrm{t},{ }^{3} \mathrm{~J}_{\mathrm{H}-\mathrm{H}}=7 \mathrm{~Hz}\right.$, pentane-Me). ${ }^{13} \mathrm{C}\left\{{ }^{1} \mathrm{H}\right\} \mathrm{NMR}(126 \mathrm{MHz}$, $\left.\mathrm{CDCl}_{3}\right): 165.0\left(\mathrm{~d}, J_{\mathrm{C}-\mathrm{P}}=5 \mathrm{~Hz}\right), 154.1\left(\mathrm{~d}, J_{\mathrm{C}-\mathrm{P}}=19 \mathrm{~Hz}\right), 130.9\left(\mathrm{~d}, J_{\mathrm{C}-\mathrm{P}}=15 \mathrm{~Hz}\right), 130.1\left(\mathrm{~d}, J_{\mathrm{C}-\mathrm{P}}=\right.$ $15 \mathrm{~Hz}), 127.1\left(\mathrm{~d}, J_{\mathrm{C}-\mathrm{P}}=7 \mathrm{~Hz}\right), 96.2\left(\mathrm{~d}, J_{\mathrm{C}-\mathrm{P}}=58 \mathrm{~Hz}\right)($ Alkenyl- $C), 61.6\left(\mathrm{~d}, J_{\mathrm{C}-\mathrm{P}}=1 \mathrm{~Hz}\right), 53.3(\mathrm{~s})$, $53.1\left(\mathrm{~d}, J_{\mathrm{C}-\mathrm{P}}=11 \mathrm{~Hz}\right), 52.5(\mathrm{~s}), 50.3\left(\mathrm{~d}, J_{\mathrm{C}-\mathrm{P}}=31 \mathrm{~Hz}\right), 47.4(\mathrm{~s}), 43.7(\mathrm{br}), 41.2\left(\mathrm{~d}, J_{\mathrm{C}-\mathrm{P}}=10 \mathrm{~Hz}\right)$, $36.7(\mathrm{~s}), 34.4\left(\mathrm{~d}, J_{\mathrm{C}-\mathrm{P}}=11 \mathrm{~Hz}\right), 31.8\left(\mathrm{~d}, J_{\mathrm{C}-\mathrm{P}}=10 \mathrm{~Hz}\right), 31.5(\mathrm{~s}), 29.8(\mathrm{~s}), 29.4(\mathrm{~s}), 29.3\left(\mathrm{~d}, J_{\mathrm{C}-\mathrm{P}}=3\right.$ $\mathrm{Hz}), 27.5(\mathrm{~s}), 25.9\left(\mathrm{~d}, J_{\mathrm{C}-\mathrm{P}}=10 \mathrm{~Hz}\right), 22.6\left(\mathrm{~d}, J_{\mathrm{C}-\mathrm{P}}=6 \mathrm{~Hz}\right), 21.6\left(\mathrm{~d}, J_{\mathrm{C}-\mathrm{P}}=4 \mathrm{~Hz}\right), 19.1\left(\mathrm{~d}, J_{\mathrm{C}-\mathrm{P}}=3\right.$ $\mathrm{Hz}), 10.3\left(\mathrm{~d}, J_{\mathrm{C}-\mathrm{P}}=54 \mathrm{~Hz}\right)($ Saturated $C), 113.3,48.6,22.1(\operatorname{IPr}=\mathrm{S}-C), 34.3,22.5,14.2$ (pentane-C). ${ }^{31} \mathrm{P}$ NMR $\left(162 \mathrm{MHz}, \mathrm{CDCl}_{3}\right): \delta(\mathrm{ppm}) 60.2\left(\mathrm{dm}, \mathrm{JP}_{\mathrm{P}-\mathrm{H}}=21 \mathrm{~Hz}\right) .{ }^{31} \mathrm{P}\left\{{ }^{1} \mathrm{H}\right\} \mathrm{NMR}$ $\left(162 \mathrm{MHz}, \mathrm{CDCl}_{3}\right): \delta(\mathrm{ppm}) 60.2$ (s). MS (ESI) $[\mathrm{M}+1] \mathrm{C}_{34} \mathrm{H}_{53} \mathrm{NPS}^{+}$calc. $538.3631 \mathrm{~m} / \mathrm{z}$, found $538.3628 \mathrm{~m} / \mathrm{z}$.

Synthesis of 7. MeOTf (1 drop, excess) was added into a pentane solution of freshly generated 2 ( $9.3 \mathrm{mg}, 0.0183 \mathrm{mmol}$ ), affording a suspension. After stirring for $5 \mathrm{~min}$, the solvent was removed in vacuo to give 7 as a white solid (12.1 mg, $99 \%$ ). Colorless single crystals of 7 were grown in a 1,2-difluorobenzene solution of 7 layered with pentane at room temperature. ${ }^{1} \mathrm{H}\left\{{ }^{31} \mathrm{P}\right\}$ NMR $\left(400 \mathrm{MHz}, \mathrm{CDCl}_{3}\right): \delta(\mathrm{ppm}) 6.64\left(\mathrm{dd},{ }^{3} \mathrm{~J}_{\mathrm{H}-\mathrm{H}}=8 \mathrm{~Hz},{ }^{3} \mathrm{JH}_{\mathrm{H}-\mathrm{H}}=7 \mathrm{~Hz}, 1 \mathrm{H}\right.$, Alkenyl- $\left.H\right), 6.10(\mathrm{dd}$, ${ }^{3} J_{\mathrm{H}-\mathrm{H}}=8 \mathrm{~Hz},{ }^{4} J_{\mathrm{H}-\mathrm{H}}=1 \mathrm{~Hz}, 1 \mathrm{H}$, Alkenyl- $\left.H\right), 5.30\left(\mathrm{~s}, \mathrm{CH}_{2} \mathrm{Cl}_{2}\right), 4.02\left(\mathrm{~m}, 1 \mathrm{H}, \mathrm{PCH}_{2} \mathrm{CH}\right), 3.05$ (sept, ${ }^{3} \mathrm{H}_{\mathrm{H}-\mathrm{H}}=7 \mathrm{~Hz}, 1 \mathrm{H}, \mathrm{C} / \mathrm{Me}_{2}$ ), 2.60-2.31 (m, 4H, C $/ \mathrm{Me}_{2}$ and/or $\mathrm{Ad}-\mathrm{H}$ and/or $\mathrm{C} H_{2}$ ), 2.30 (s, 3H, PMe), 2.20 (s, 2H, Ad- $H$ and/or $\mathrm{CH}_{2}$ ), 2.12-1.75 (m, 12H, CHMe ${ }_{2}$ and/or Ad$H$ and/or $\mathrm{CH}_{2}$ ), 1.74-1.60 (m, 6H, CHMe 2 and/or Ad- $H$ and/or $\left.\mathrm{CH}_{2}\right), 1.52$ (s, 3H, CMe $)$, $1.34(\mathrm{~s}, 3 \mathrm{H}, \mathrm{CMe}), 1.29\left(\mathrm{~m}\right.$, pentane- $\left.\mathrm{CH}_{2}\right), 1.23\left(\mathrm{~d},{ }^{3} \mathrm{~J}_{\mathrm{H}-\mathrm{H}}=7 \mathrm{~Hz}, 3 \mathrm{H}, \mathrm{CH} M e_{2}\right), 1.12-$ $0.98\left(\mathrm{~m}, 15 \mathrm{H}, \mathrm{CH} M e_{2}\right.$ and $\left.\mathrm{CH}_{2} M e\right), 0.88\left(\mathrm{t},{ }^{3} \mathrm{~J}_{\mathrm{H}-\mathrm{H}}=7 \mathrm{~Hz}\right.$, pentane-Me). ${ }^{1} \mathrm{H}$ NMR $(400$ $\mathrm{MHz}, \mathrm{CDCl}_{3}$ ): $\delta(\mathrm{ppm}) 6.64$ (ddd (pseudo quartet), ${ }^{3} J_{\mathrm{H}-\mathrm{H}} \approx{ }^{3} J_{\mathrm{H}-\mathrm{H}} \approx{ }^{4} J_{\mathrm{P}-\mathrm{H}}=7 \mathrm{~Hz}, 1 \mathrm{H}$, 
Alkenyl- $H$ ), $6.10\left(\mathrm{ddd},{ }^{3} \mathrm{~J}_{\mathrm{H}-\mathrm{H}}=8 \mathrm{~Hz},{ }^{3} \mathrm{JP}_{\mathrm{P}-\mathrm{H}}=4 \mathrm{~Hz},{ }^{4} \mathrm{~J}_{\mathrm{H}-\mathrm{H}}=1 \mathrm{~Hz}, 1 \mathrm{H}\right.$, Alkenyl- $H$ ), $5.30(\mathrm{~s}$, $\left.\mathrm{CH}_{2} \mathrm{Cl}_{2}\right), 4.02\left(\mathrm{dm},{ }^{3} \mathrm{JP}_{\mathrm{P}-\mathrm{H}}=23 \mathrm{~Hz}, 1 \mathrm{H}, \mathrm{PCH}_{2} \mathrm{CH}\right), 3.05$ (sept, $\left.{ }^{3} \mathrm{JH}_{\mathrm{H}-\mathrm{H}}=7 \mathrm{~Hz}, 1 \mathrm{H}, \mathrm{C} / \mathrm{Me}_{2}\right)$, 2.60-2.31 (m, 4H, CHMe 2 and/or Ad- $H$ and/or $\left.C H_{2}\right), 2.30\left(\mathrm{~d},{ }^{2} J_{\mathrm{P}-\mathrm{H}}=11 \mathrm{~Hz}, 3 \mathrm{H}, \mathrm{PMe}\right)$, $2.20\left(\mathrm{~s}, 2 \mathrm{H}, \mathrm{Ad}-\mathrm{H}\right.$ and/or $\left.\mathrm{CH}_{2}\right), 2.12-1.75\left(\mathrm{~m}, 12 \mathrm{H}, \mathrm{C} / \mathrm{Me}_{2}\right.$ and/or $\mathrm{Ad}-\mathrm{H}$ and/or $\mathrm{CH}_{2}$ ), 1.74-1.60 (m, 6H, CHMe 2 and/or Ad- $H$ and/or $\left.\mathrm{CH}_{2}\right), 1.52(\mathrm{~s}, 3 \mathrm{H}, \mathrm{CMe}$ ), $1.34(\mathrm{~s}, 3 \mathrm{H}$, $\left.\mathrm{C} M e_{2}\right), 1.29\left(\mathrm{~m}\right.$, pentane- $\left.\mathrm{C}_{2}\right), 1.23\left(\mathrm{~d},{ }^{3} \mathrm{~J}_{\mathrm{H}-\mathrm{H}}=7 \mathrm{~Hz}, 3 \mathrm{H}, \mathrm{CH} M e_{2}\right), 1.12-0.98(\mathrm{~m}, 15 \mathrm{H}$, $\mathrm{CHMe}$ and $\left.\mathrm{CH}_{2} \mathrm{Me}\right), 0.88\left(\mathrm{t},{ }^{3} \mathrm{~J}_{\mathrm{H}-\mathrm{H}}=7 \mathrm{~Hz}\right.$, pentane-Me). ${ }^{13} \mathrm{C}\left\{{ }^{1} \mathrm{H}\right\} \mathrm{NMR}(126 \mathrm{MHz}$, $\left.\mathrm{CDCl}_{3}\right): \delta(\mathrm{ppm}) 172.9(\mathrm{~m}), 161.0\left(\mathrm{~d}, J_{\mathrm{C}-\mathrm{P}}=18 \mathrm{~Hz}\right), 135.4\left(\mathrm{~d}, J_{\mathrm{C}-\mathrm{P}}=14 \mathrm{~Hz}\right), 127.9\left(\mathrm{~d}, J_{\mathrm{C}-\mathrm{P}}=17\right.$ $\mathrm{Hz}), 123.0\left(\mathrm{~d}, J_{\mathrm{C}-\mathrm{P}}=8 \mathrm{~Hz}\right), 84.2\left(\mathrm{~d}, J_{\mathrm{C}-\mathrm{P}}=68 \mathrm{~Hz}\right)($ Alkenyl- $C), 121.1\left(\mathrm{q},{ }^{1} J_{\mathrm{C}-\mathrm{F}}=321 \mathrm{~Hz}\right.$, $\left.\mathrm{OSO}_{2} \mathrm{CF}_{3}\right), 63.8(\mathrm{~s}), 53.3(\mathrm{~s}), 55.0\left(\mathrm{~d}, J_{\mathrm{C}-\mathrm{P}}=12 \mathrm{~Hz}\right), 47.0(\mathrm{~s}), 45.1(\mathrm{~s}), 44.9\left(\mathrm{~d}, J_{\mathrm{C}-\mathrm{P}}=6 \mathrm{~Hz}\right), 44.4$ $\left(\mathrm{d}, J_{\mathrm{C}-\mathrm{P}}=16 \mathrm{~Hz}\right), 44.2(\mathrm{~s}), 40.5\left(\mathrm{~d}, J_{\mathrm{C}-\mathrm{P}}=9 \mathrm{~Hz}\right), 36.0(\mathrm{~s}), 34.2\left(\mathrm{~d}, J_{\mathrm{C}-\mathrm{P}}=10 \mathrm{~Hz}\right), 31.6(\mathrm{~s}), 31.5(\mathrm{~d}$, $\left.J_{\mathrm{C}-\mathrm{P}}=1 \mathrm{~Hz}\right), 31.3(\mathrm{~s}), 29.99(\mathrm{~s}), 29.96\left(\mathrm{~d}, J_{\mathrm{C}-\mathrm{P}}=2 \mathrm{~Hz}\right), 29.2(\mathrm{~s}), 26.6(\mathrm{~s}), 24.5\left(\mathrm{~d}, J_{\mathrm{C}-\mathrm{P}}=10 \mathrm{~Hz}\right)$, $22.3\left(\mathrm{~d}, J_{\mathrm{C}-\mathrm{P}}=6 \mathrm{~Hz}\right), 21.4\left(\mathrm{~d}, J_{\mathrm{C}-\mathrm{P}}=4 \mathrm{~Hz}\right), 20.6\left(\mathrm{~d}, J_{\mathrm{C}-\mathrm{P}}=41 \mathrm{~Hz}\right), 18.9\left(\mathrm{~d}, J_{\mathrm{C}-\mathrm{P}}=2 \mathrm{~Hz}\right), 10.3(\mathrm{~d}$, $\left.J_{\mathrm{C}-\mathrm{P}}=60 \mathrm{~Hz}\right)\left(\right.$ Saturated C), 34.3, 22.5, 14.2 (pentane-C). ${ }^{19} \mathrm{~F}\left\{{ }^{1} \mathrm{H}\right\}$ NMR $\left(162 \mathrm{MHz}, \mathrm{CDCl}_{3}\right): \delta$ (ppm) -78.2 (s, $\left.\mathrm{OSO}_{2} \mathrm{CF}_{3}\right) .{ }^{31} \mathrm{P}$ NMR $\left(162 \mathrm{MHz}, \mathrm{CDCl}_{3}\right): \delta(\mathrm{ppm}) 29.7(\mathrm{~m}) .{ }^{31} \mathrm{P}\left\{{ }^{1} \mathrm{H}\right\} \mathrm{NMR}(162$ $\mathrm{MHz}, \mathrm{CDCl}_{3}$ ): $\delta(\mathrm{ppm}) 29.7$ (s). MS (ESI) [M] $\mathrm{C}_{35} \mathrm{H}_{55} \mathrm{NP}^{+}$calc. $520.4067 \mathrm{~m} / \mathrm{z}$, found 520.4079 $\mathrm{m} / \mathrm{z}$.

\section{Copies of NMR Spectra}




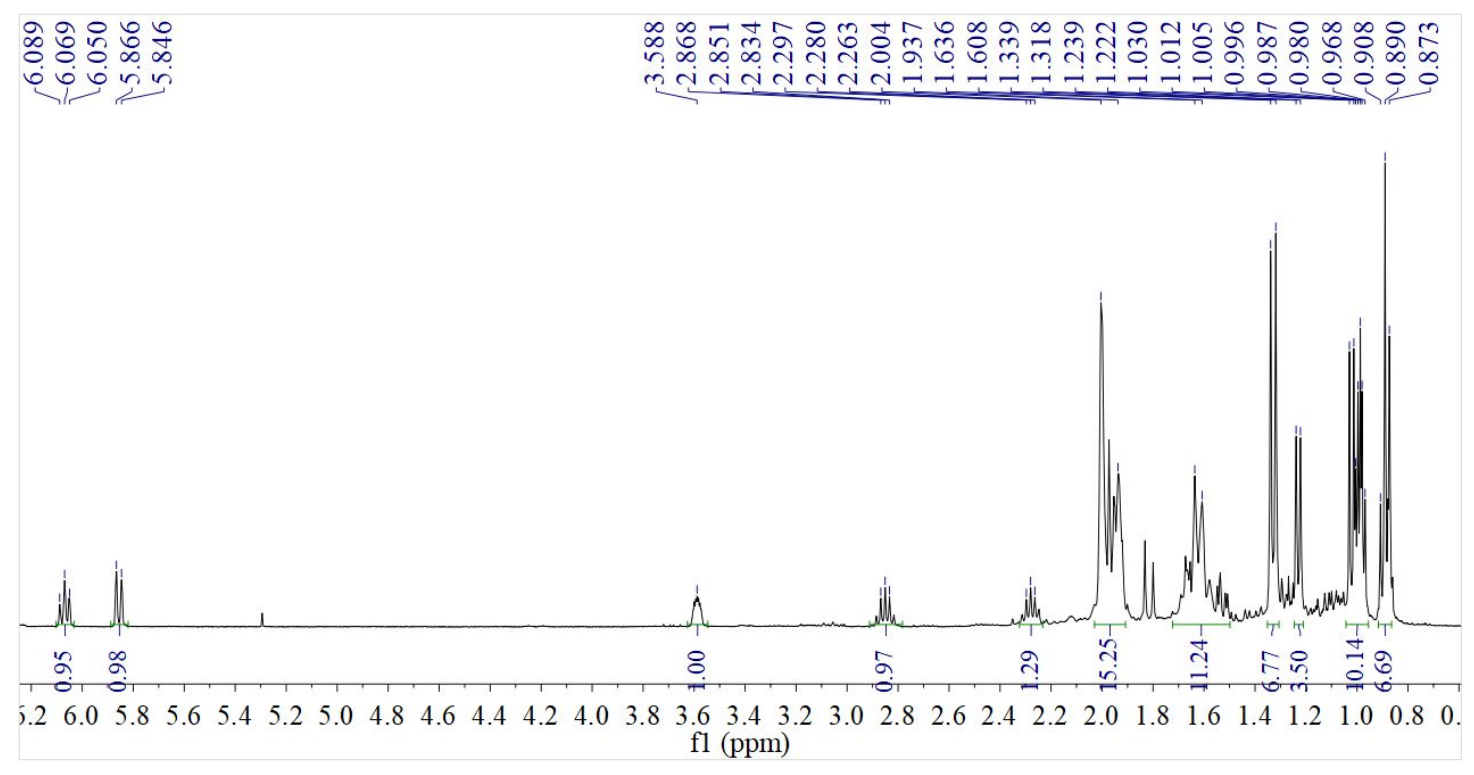

Figure $\mathrm{S} 1 .{ }^{1} \mathrm{H}\left\{{ }^{31} \mathrm{P}\right\} \mathrm{NMR}$ spectrum of $2\left(400 \mathrm{MHz}, \mathrm{CDCl}_{3}\right)$.

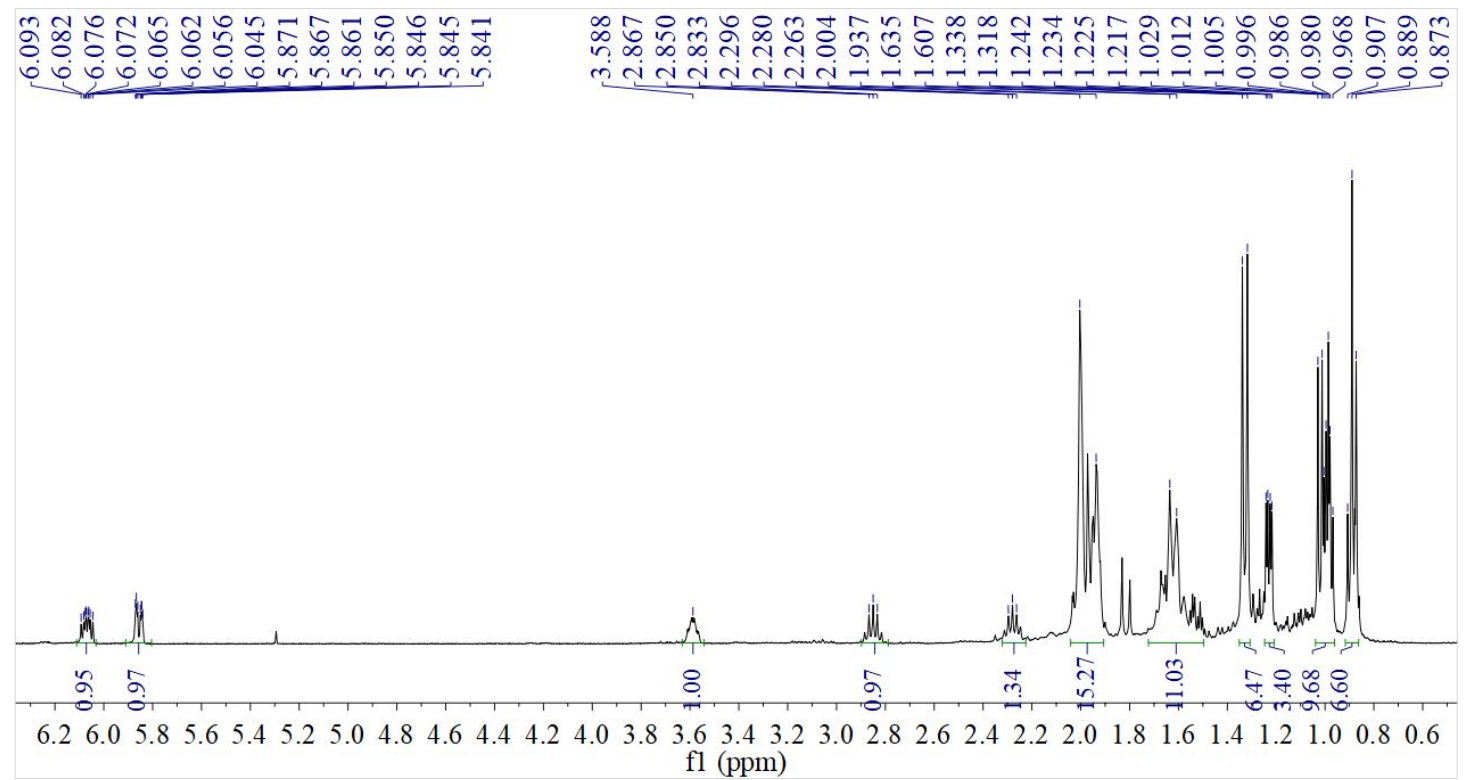

Figure S2. ${ }^{1} \mathrm{H}$ NMR spectrum of $2\left(400 \mathrm{MHz}, \mathrm{CDCl}_{3}\right)$. 


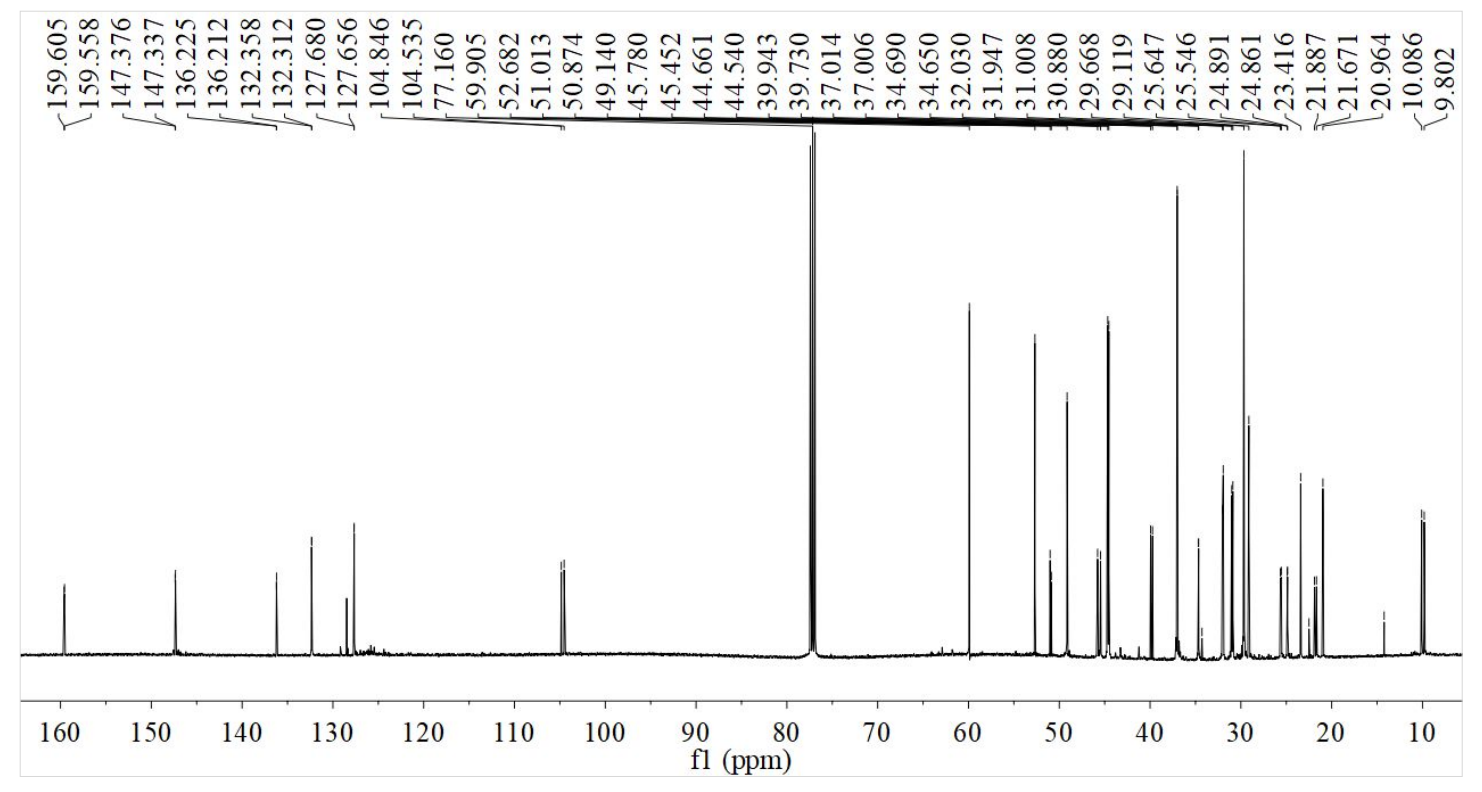

Figure S3. ${ }^{13} \mathrm{C}\left\{{ }^{1} \mathrm{H}\right\}$ NMR spectrum of $2\left(126 \mathrm{MHz}, \mathrm{CDCl}_{3}\right)$.

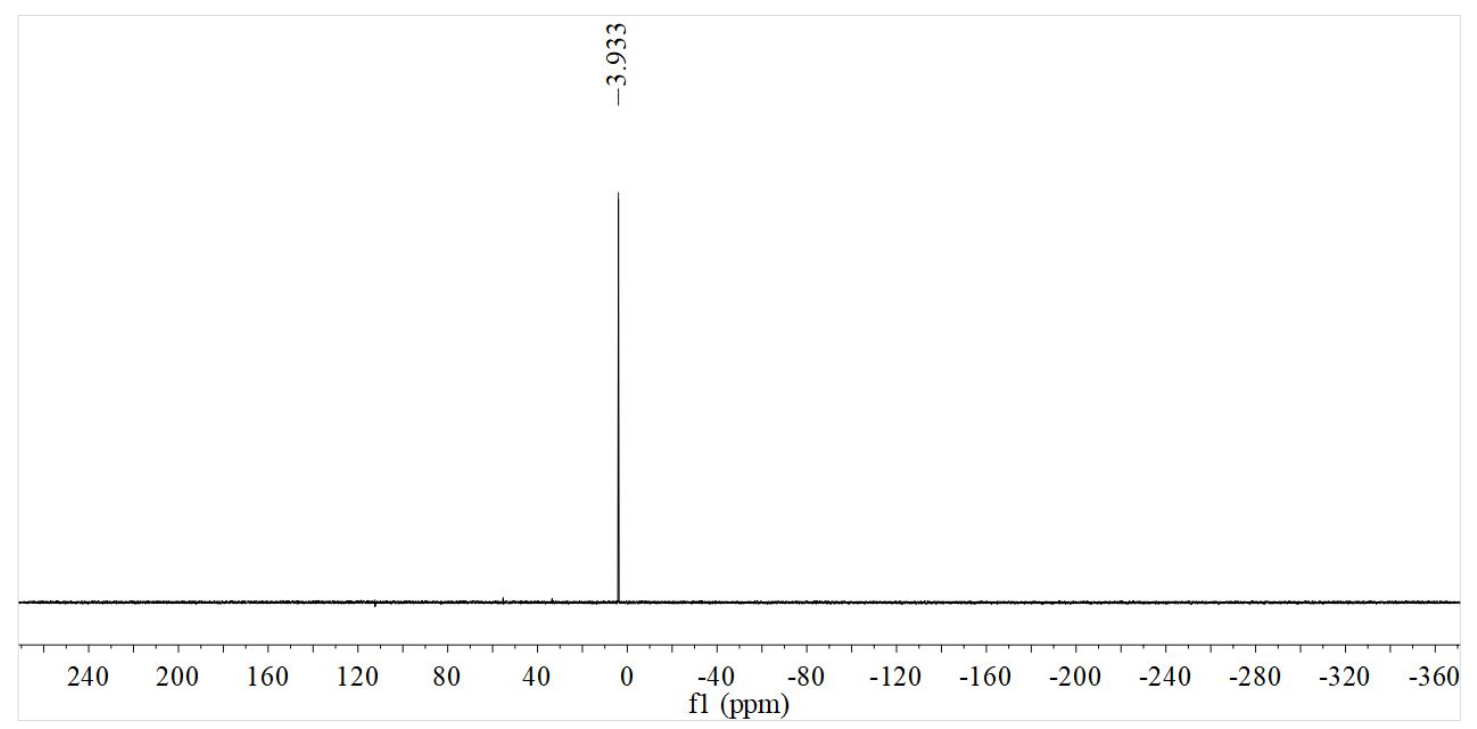

Figure S4. ${ }^{31} \mathrm{P}\left\{{ }^{1} \mathrm{H}\right\}$ NMR spectrum of $2\left(162 \mathrm{MHz}, \mathrm{CDCl}_{3}\right)$. 


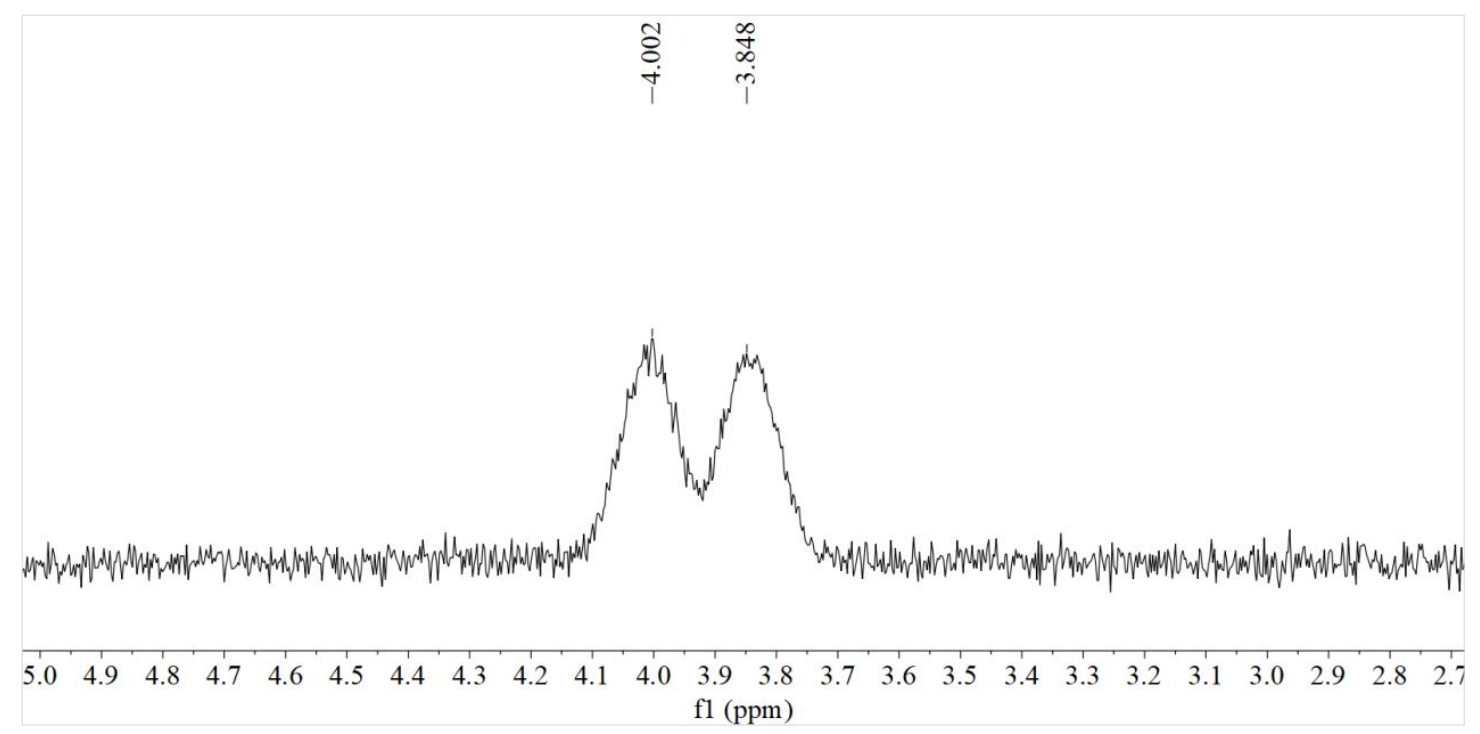

Figure S5. ${ }^{31} \mathrm{P}$ NMR spectrum of $2\left(162 \mathrm{MHz}, \mathrm{CDCl}_{3}\right)$.

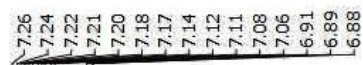

管

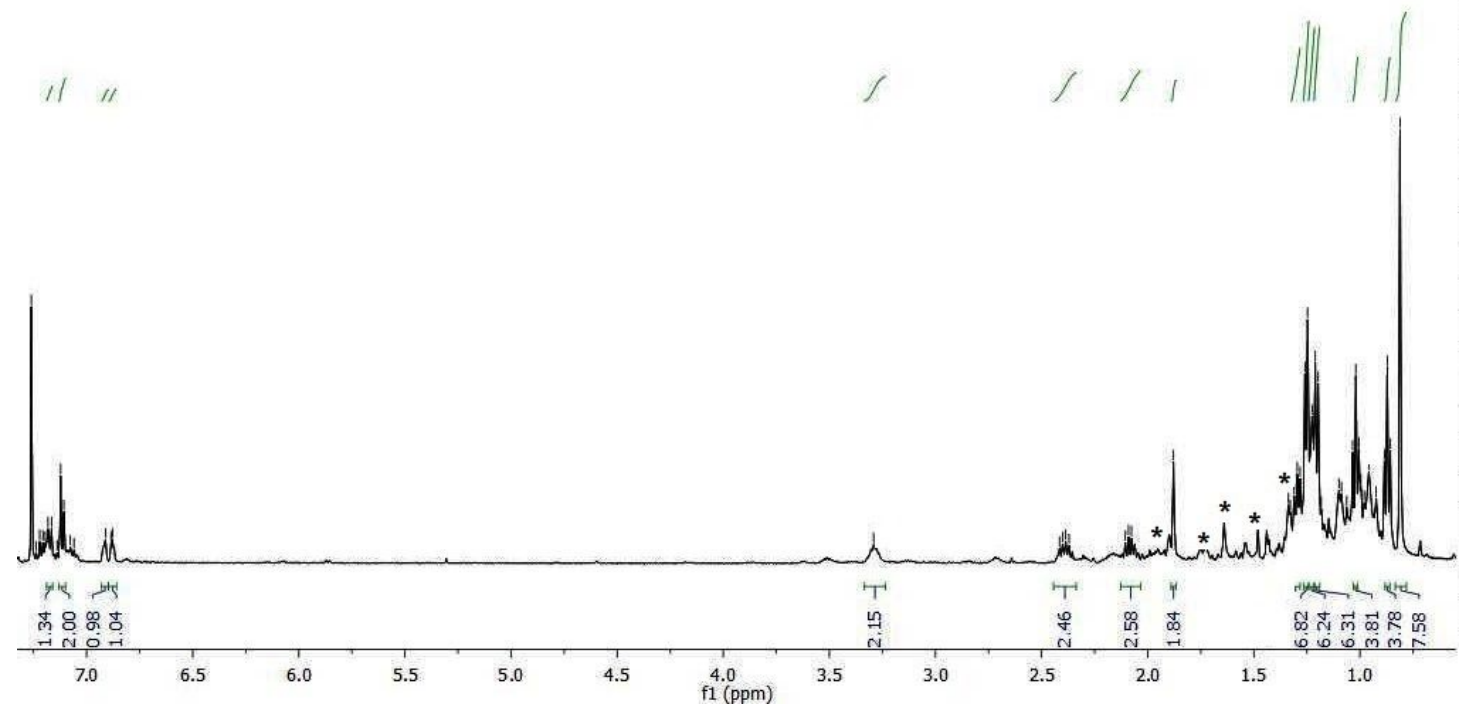

Figure S6. ${ }^{1} \mathrm{H}\left\{{ }^{31} \mathrm{P}\right\}$ NMR spectrum of $4\left(500 \mathrm{MHz}, \mathrm{CDCl}_{3}\right)$. Asterisk denotes unidentified impurity. 


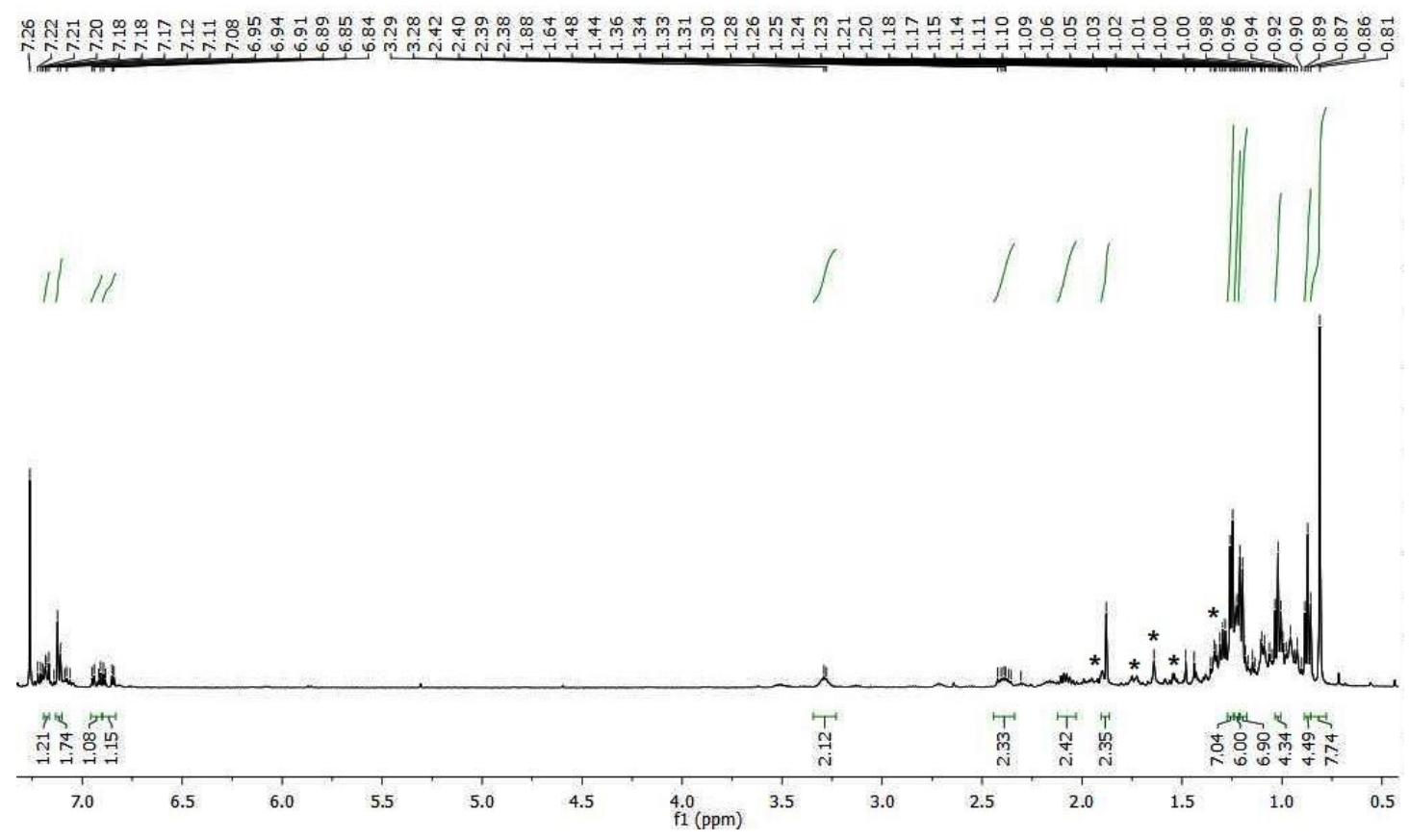

Figure S7. ${ }^{1} \mathrm{H}$ NMR spectrum of $4\left(500 \mathrm{MHz}, \mathrm{CDCl}_{3}\right)$. Asterisk denotes unidentified impurity.

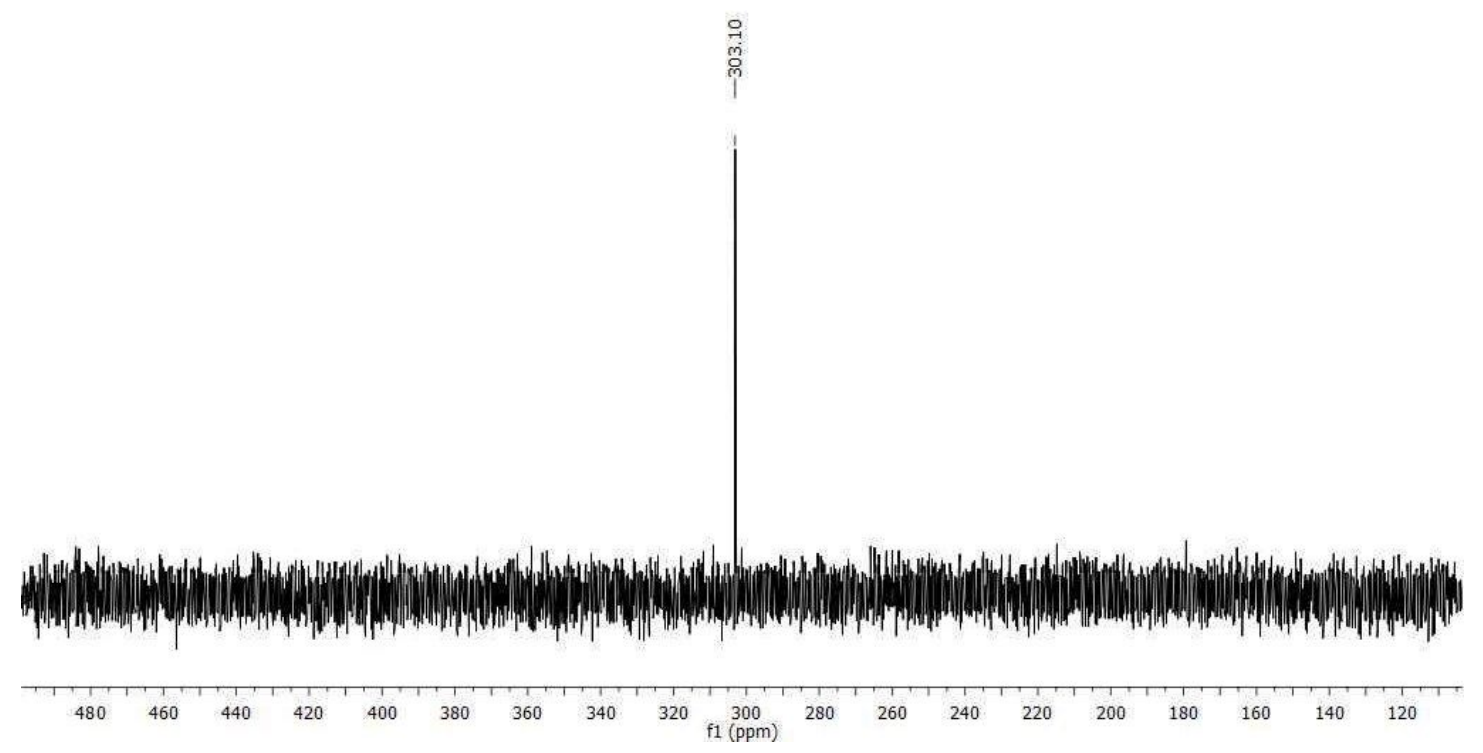

Figure S8. ${ }^{31} \mathrm{P}\left\{{ }^{1} \mathrm{H}\right\}$ NMR spectrum of $4\left(162 \mathrm{MHz}, \mathrm{C}_{6} \mathrm{D}_{6}\right)$. 


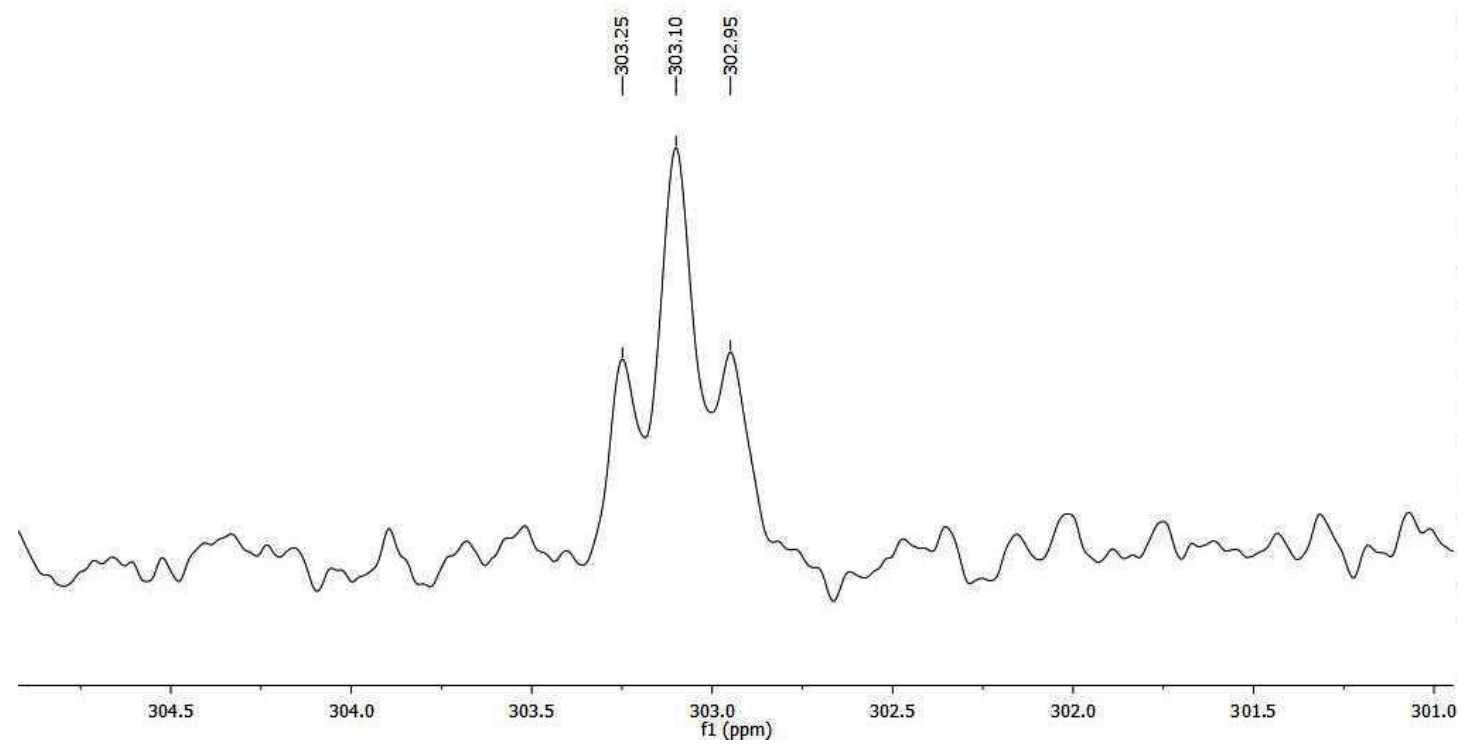

Figure S9. ${ }^{31} \mathrm{P}$ NMR spectrum of $4\left(162 \mathrm{MHz}, \mathrm{C}_{6} \mathrm{D}_{6}\right)$.

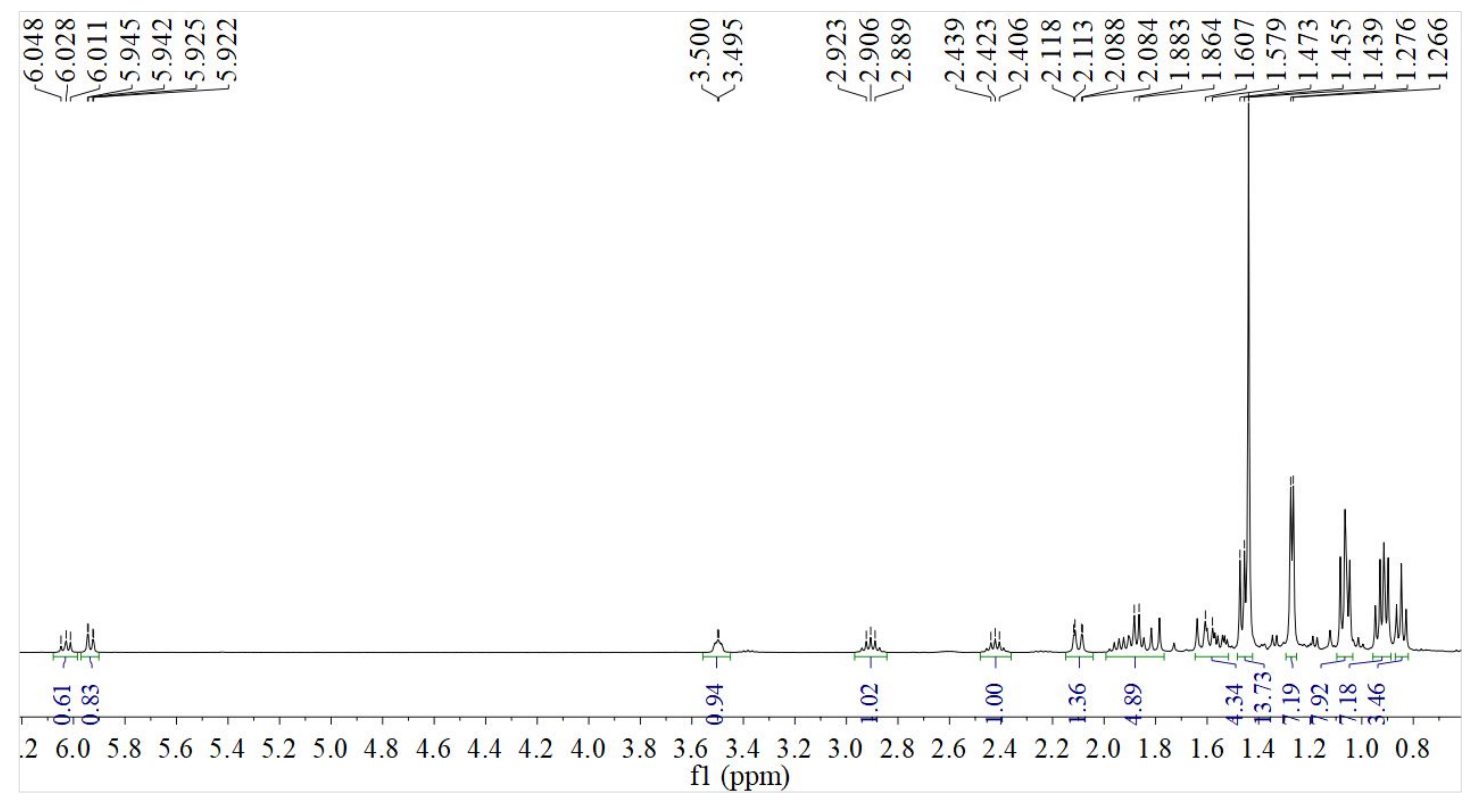

Figure $\mathrm{S} 10 .{ }^{1} \mathrm{H}\left\{{ }^{31} \mathrm{P}\right\}$ NMR spectrum of $5\left(400 \mathrm{MHz}, \mathrm{C}_{6} \mathrm{D}_{6}\right)$. 


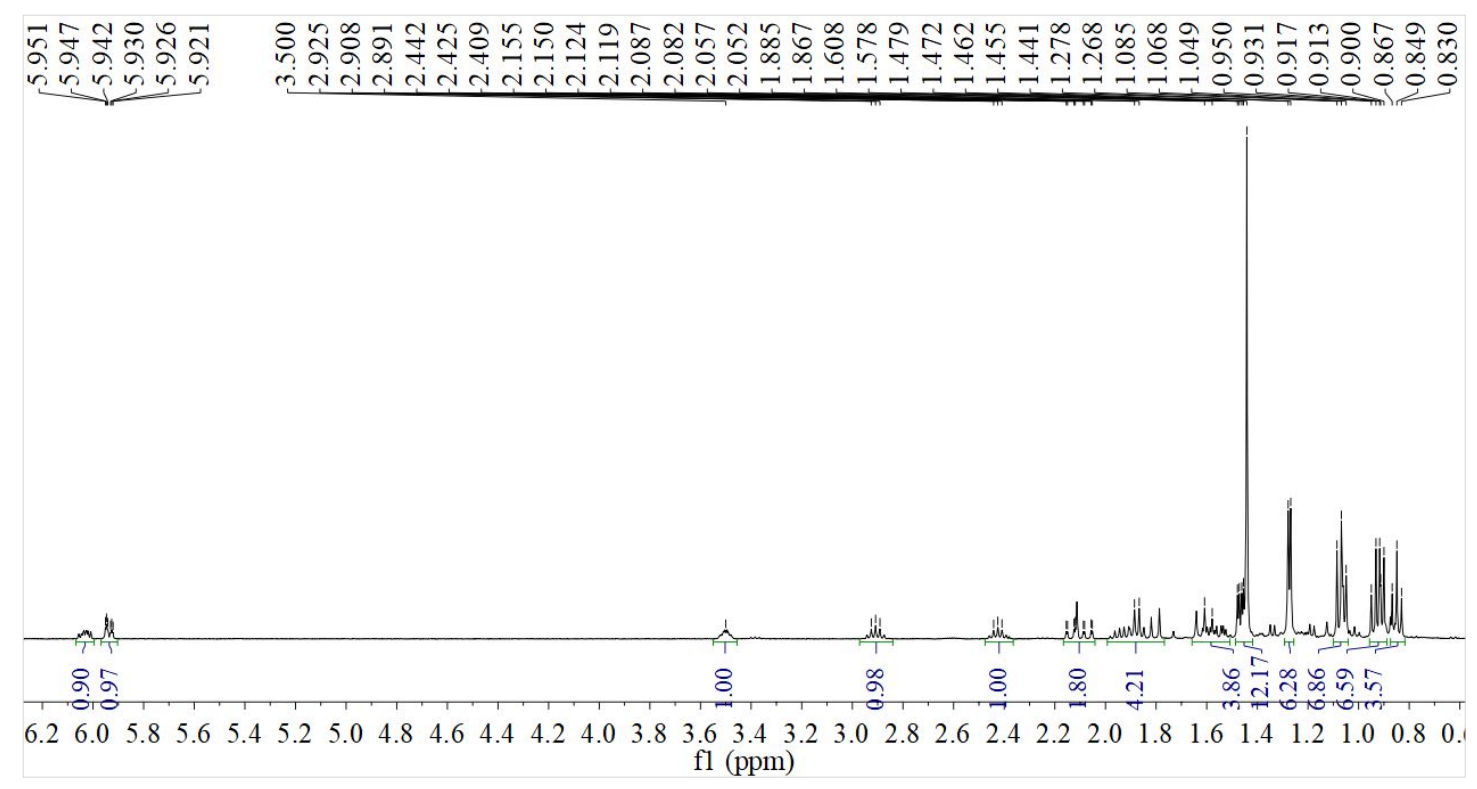

Figure S11. ${ }^{1} \mathrm{H}$ NMR spectrum of $5\left(400 \mathrm{MHz}, \mathrm{C}_{6} \mathrm{D}_{6}\right)$.

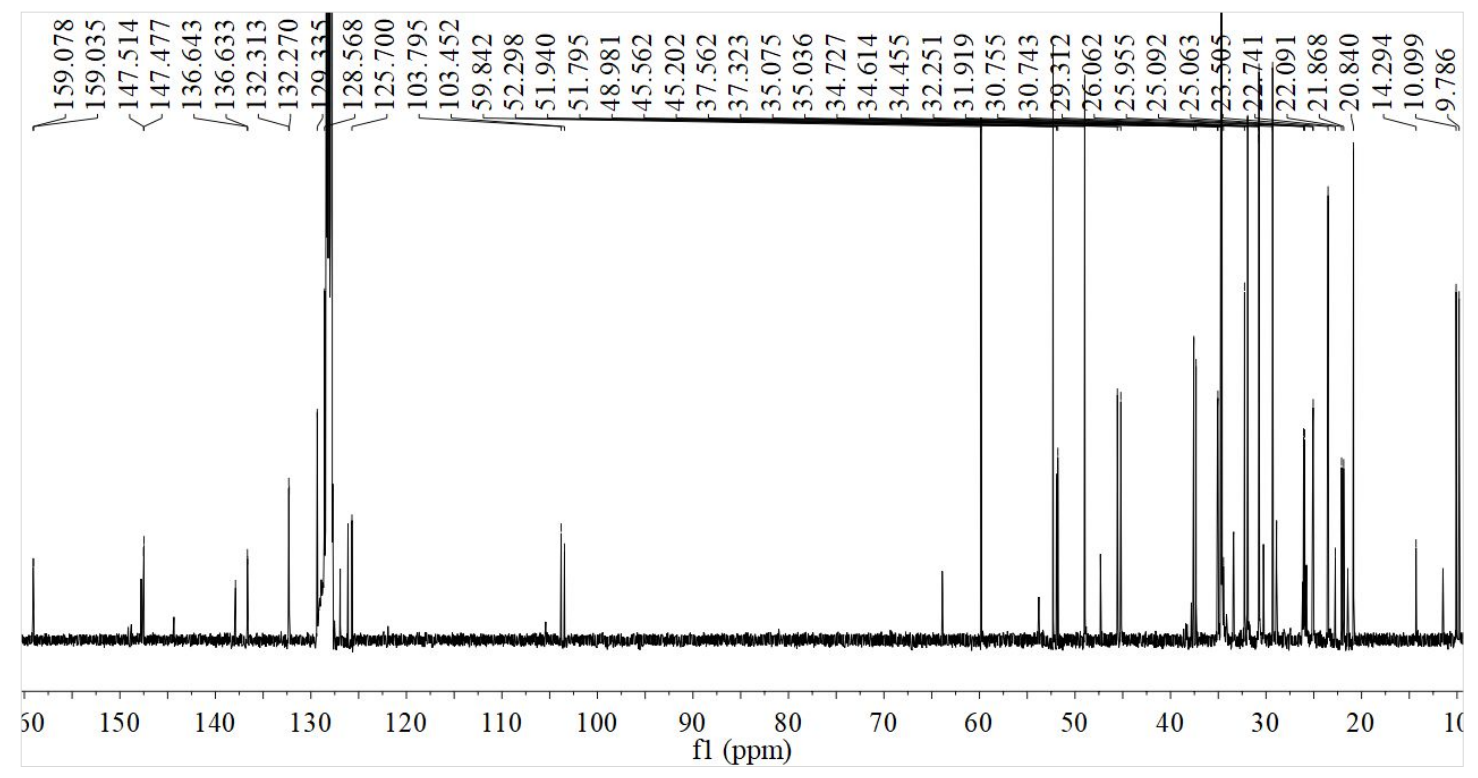

Figure $\mathrm{S} 12 .{ }^{13} \mathrm{C}\left\{{ }^{1} \mathrm{H}\right\}$ NMR spectrum of $5\left(126 \mathrm{MHz}, \mathrm{C}_{6} \mathrm{D}_{6}\right)$. 


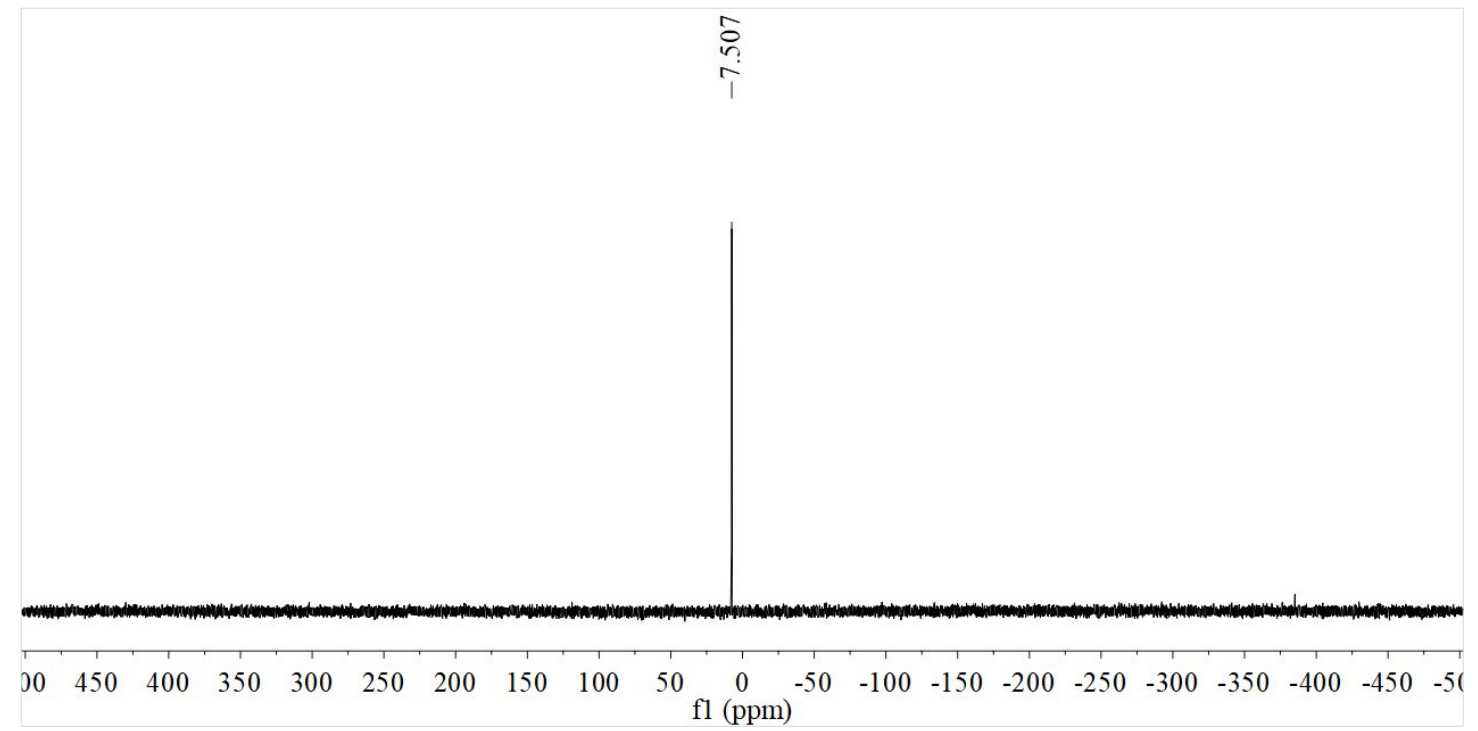

Figure $\mathrm{S} 13 .{ }^{31} \mathrm{P}\left\{{ }^{1} \mathrm{H}\right\}$ NMR spectrum of $5\left(162 \mathrm{MHz}, \mathrm{C}_{6} \mathrm{D}_{6}\right)$.

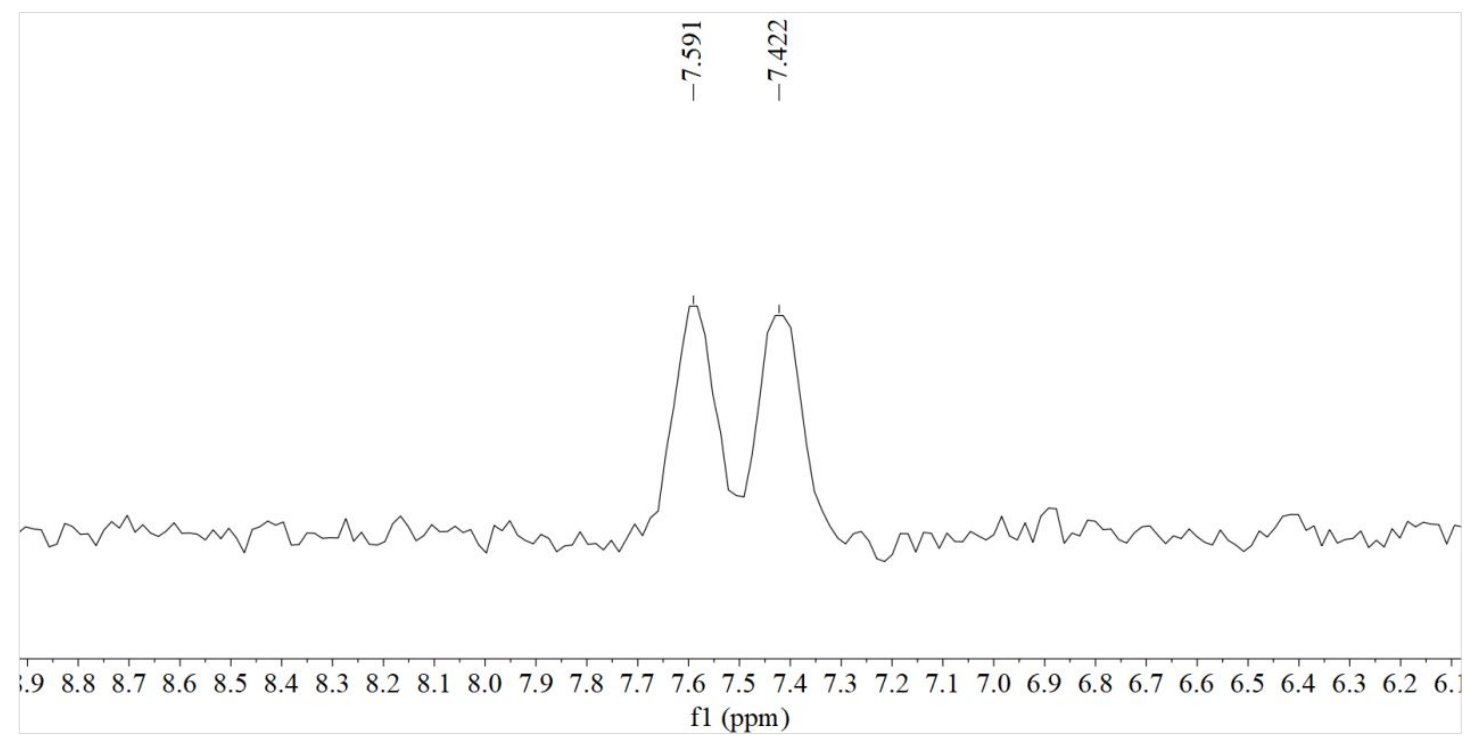

Figure S14. ${ }^{31} \mathrm{P}$ NMR spectrum of $5\left(162 \mathrm{MHz}, \mathrm{C}_{6} \mathrm{D}_{6}\right)$. 


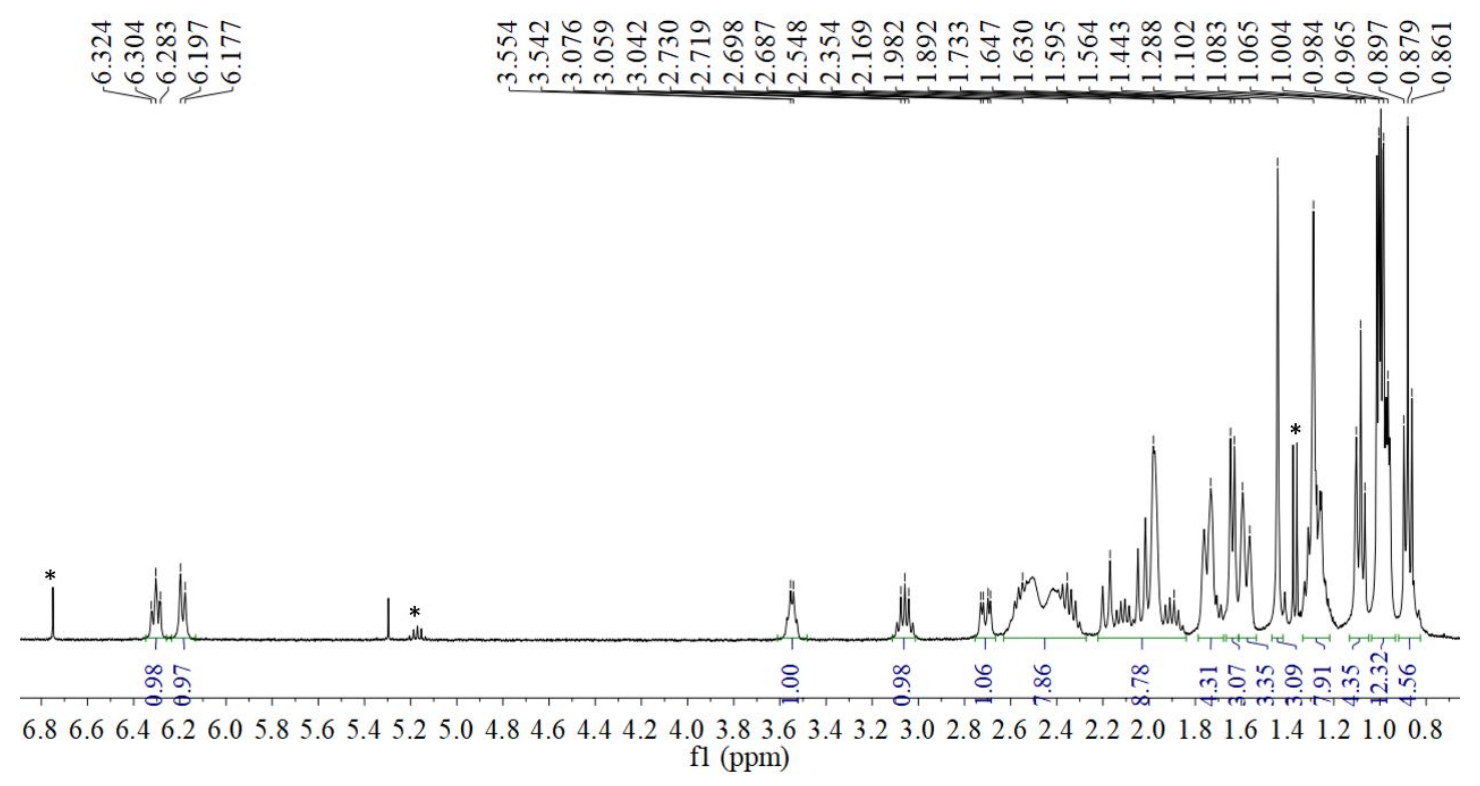

Figure S15. ${ }^{1} \mathrm{H}\left\{{ }^{31} \mathrm{P}\right\}$ NMR spectrum of $6\left(400 \mathrm{MHz}, \mathrm{CDCl}_{3}\right)$. Asterisk denotes $\mathrm{IPr}=\mathrm{S}$.

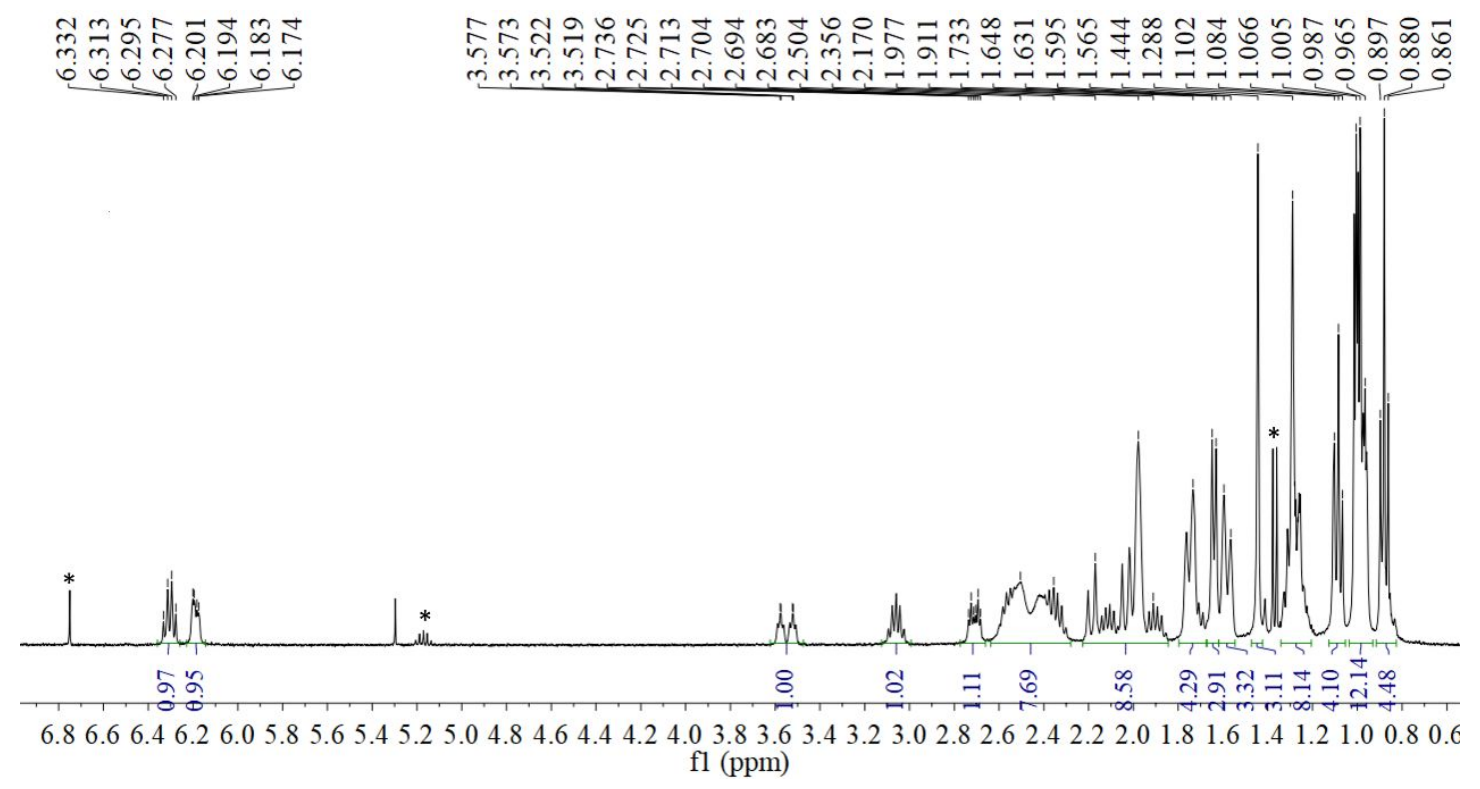

Figure S16. ${ }^{1} \mathrm{H}$ NMR spectrum of $6\left(400 \mathrm{MHz}, \mathrm{CDCl}_{3}\right)$. Asterisk denotes IPr=S. 


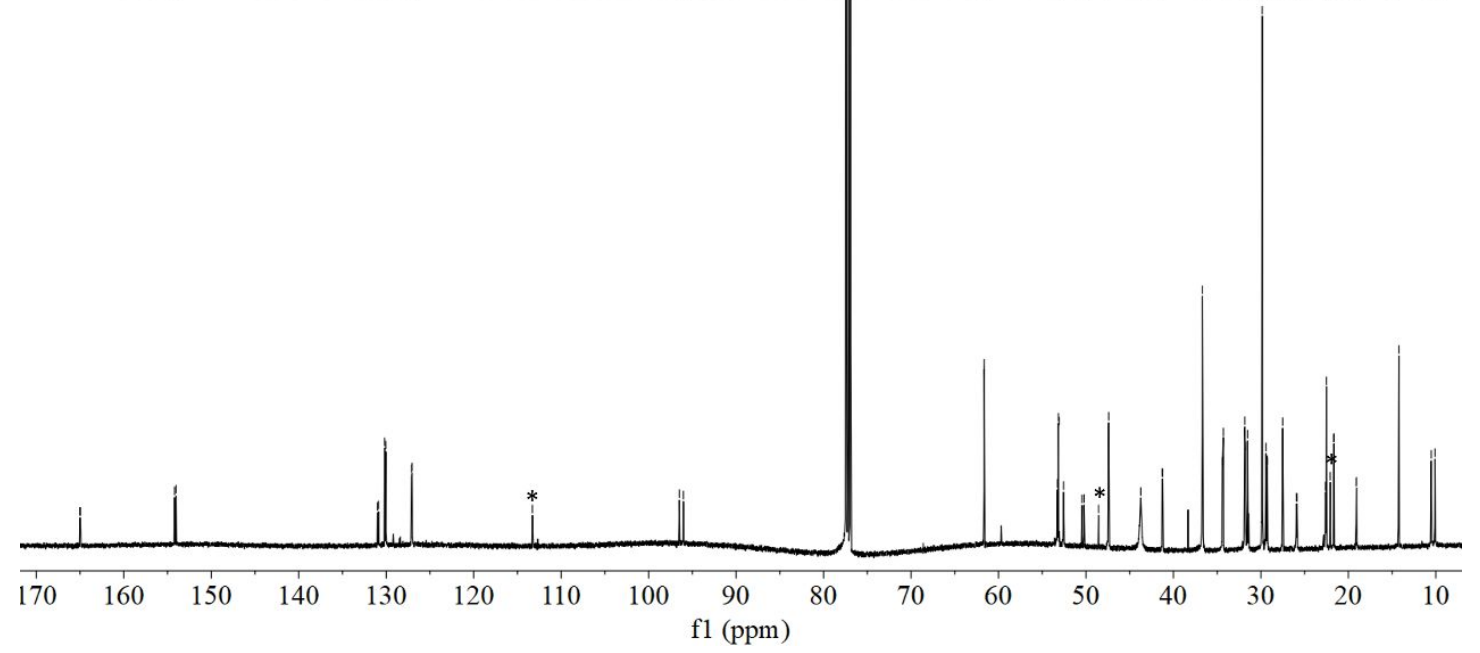

Figure S17. ${ }^{13} \mathrm{C}\left\{{ }^{1} \mathrm{H}\right\}$ NMR spectrum of $6\left(126 \mathrm{MHz}, \mathrm{CDCl}_{3}\right)$. Asterisk denotes IPr=S.

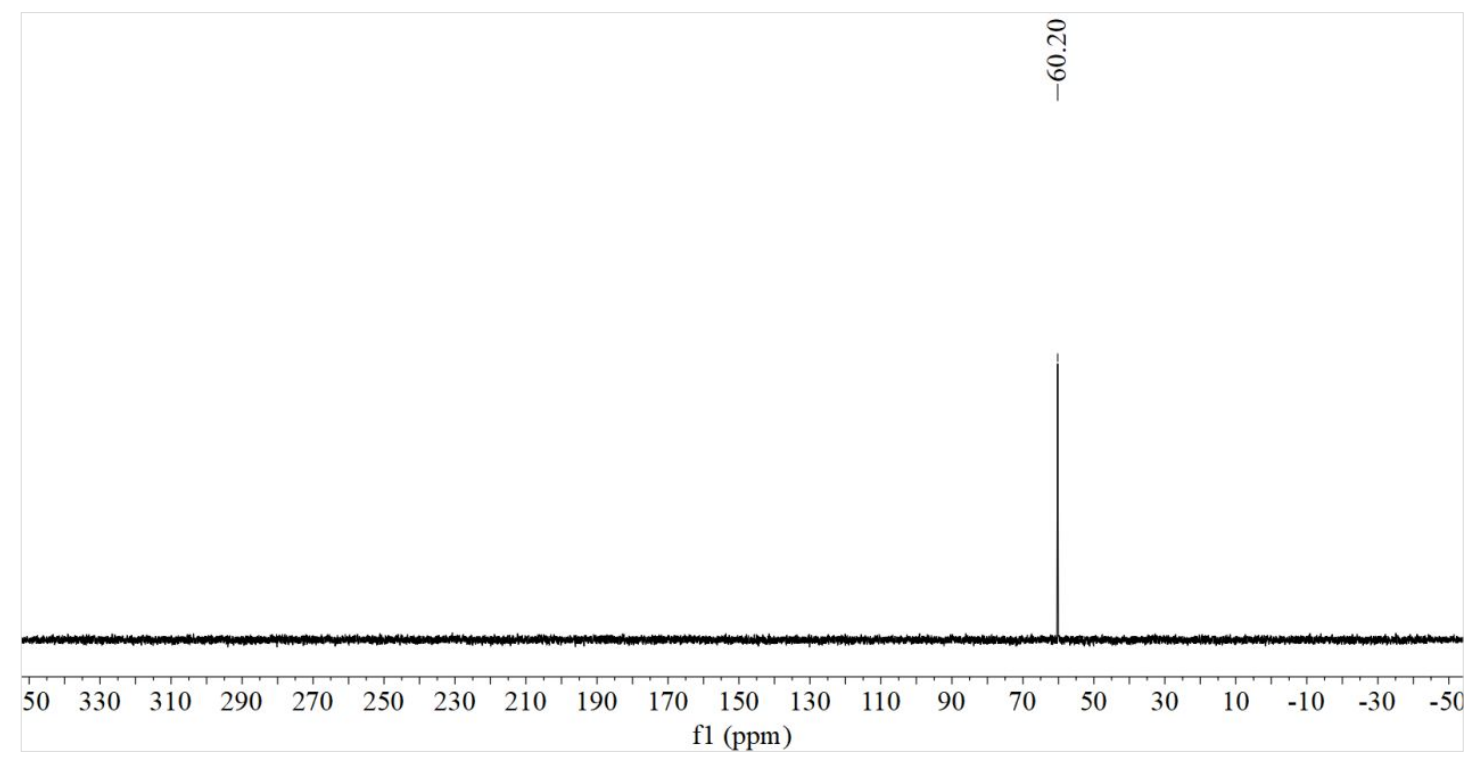

Figure S18. ${ }^{31} \mathrm{P}\left\{{ }^{1} \mathrm{H}\right\}$ NMR spectrum of $6\left(162 \mathrm{MHz}, \mathrm{CDCl}_{3}\right)$. 


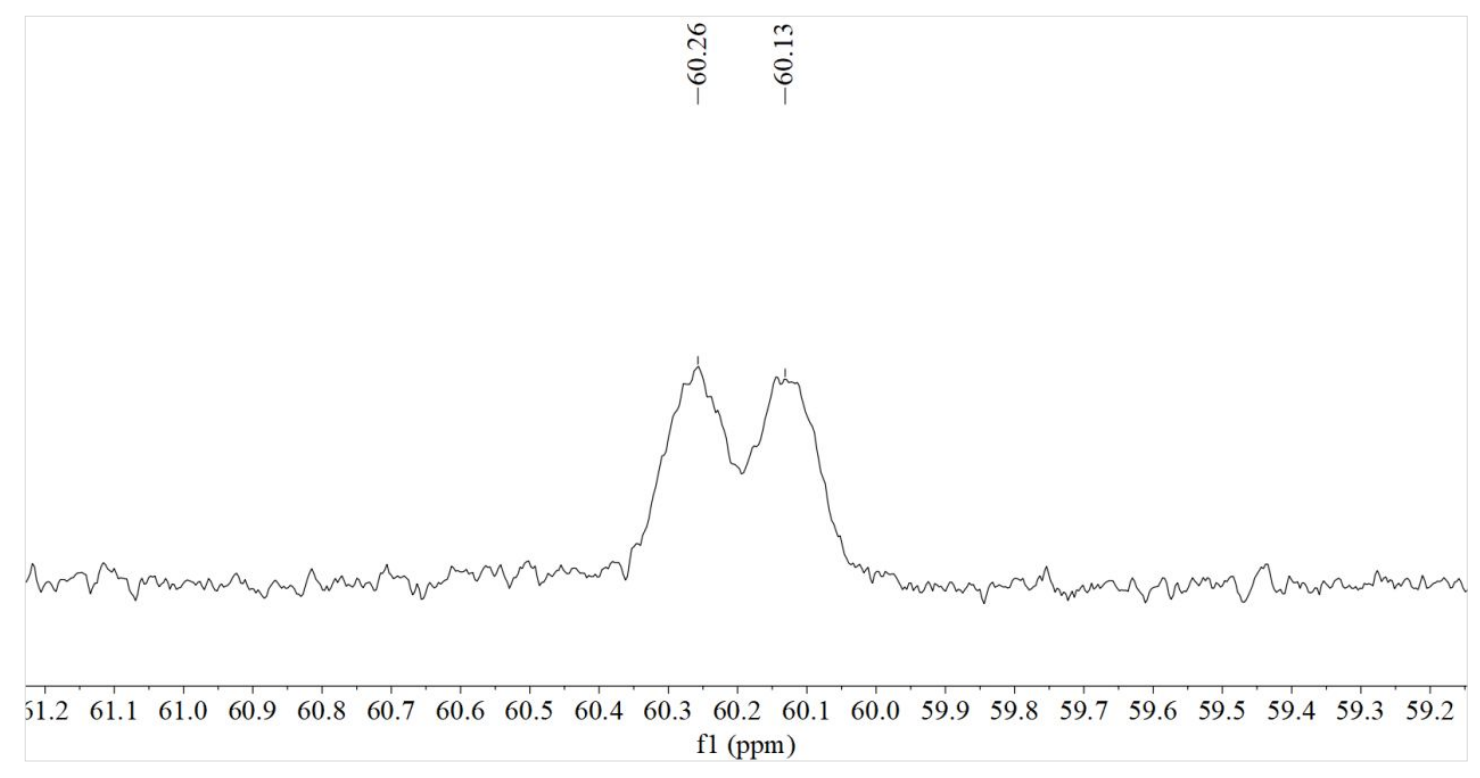

Figure S19. ${ }^{31} \mathrm{P}$ NMR spectrum of $6\left(162 \mathrm{MHz}, \mathrm{CDCl}_{3}\right)$.

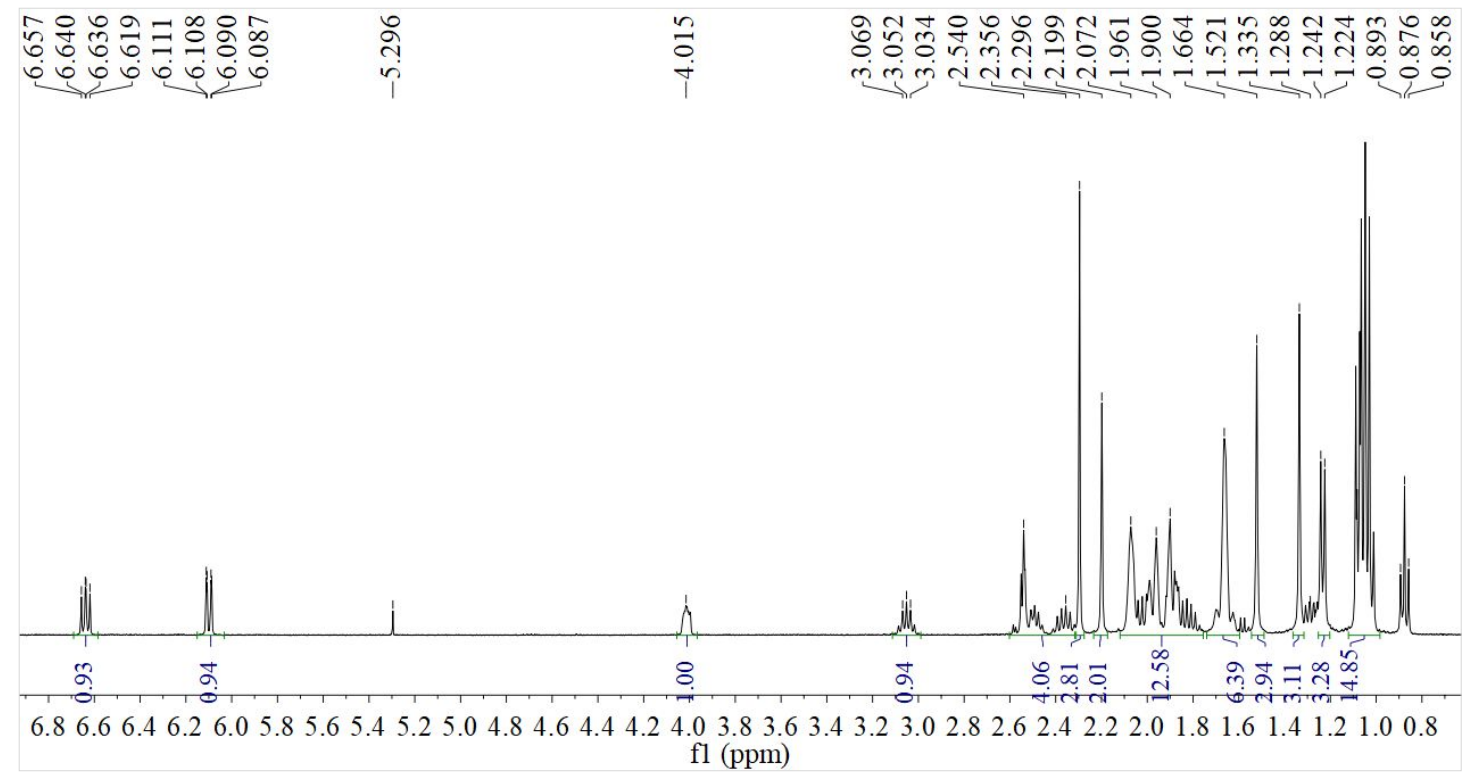

Figure S20. ${ }^{1} \mathrm{H}\left\{{ }^{31} \mathrm{P}\right\} \mathrm{NMR}$ spectrum of $7\left(400 \mathrm{MHz}, \mathrm{CDCl}_{3}\right)$. 


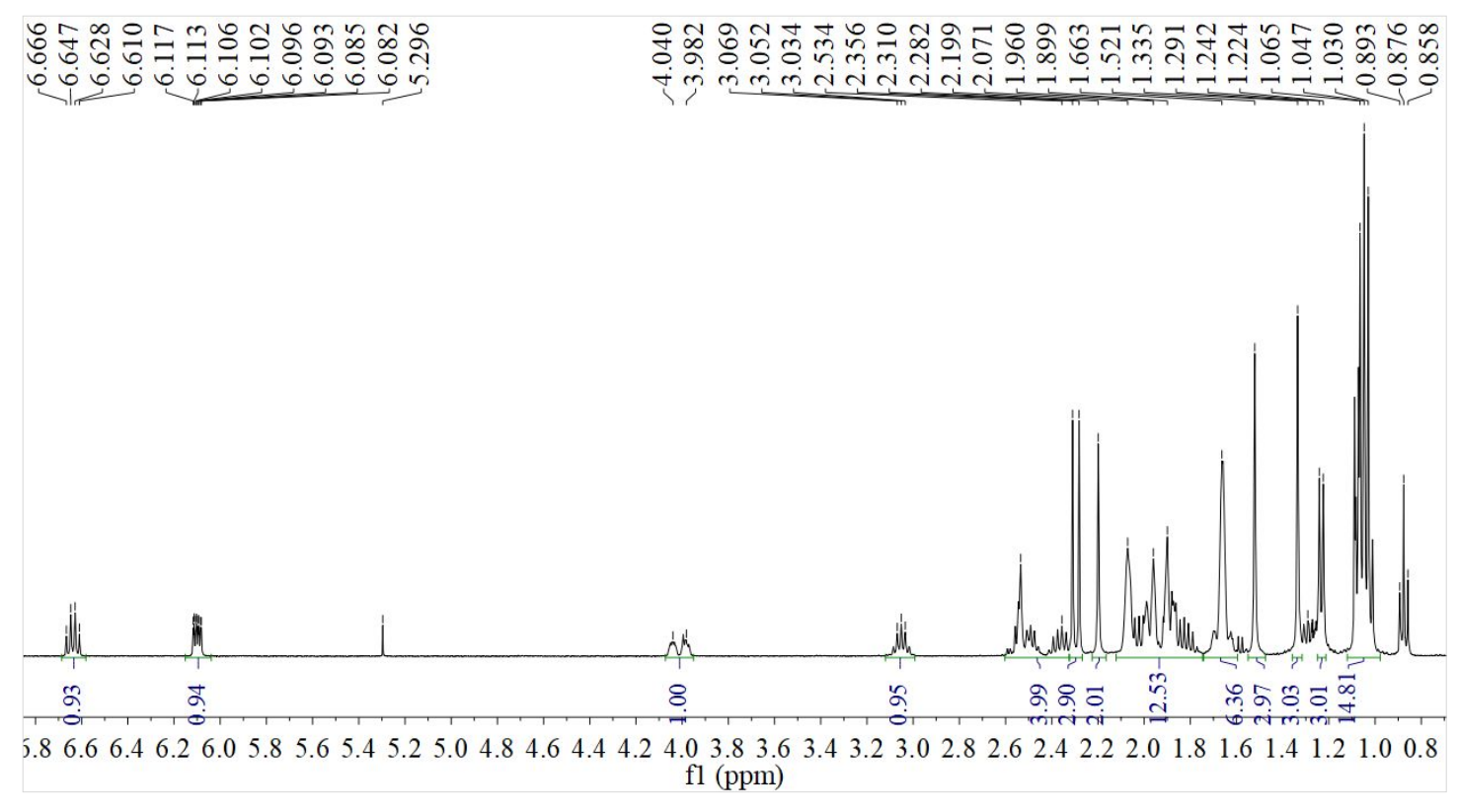

Figure S21. ${ }^{1} \mathrm{H}$ NMR spectrum of $7\left(400 \mathrm{MHz}, \mathrm{CDCl}_{3}\right)$.

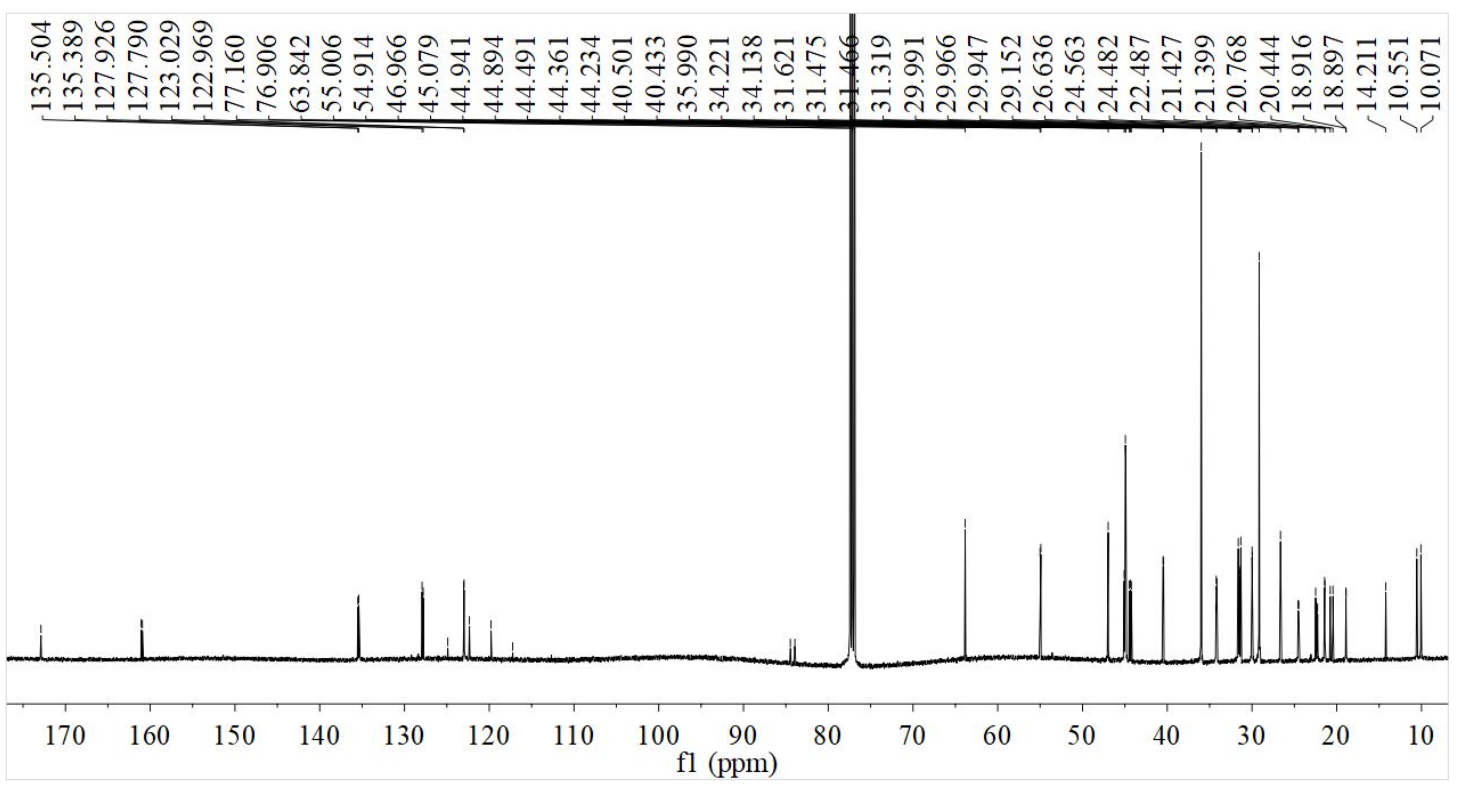

Figure S22. ${ }^{13} \mathrm{C}\left\{{ }^{1} \mathrm{H}\right\}$ NMR spectrum of $7\left(126 \mathrm{MHz}, \mathrm{CDCl}_{3}\right)$. 


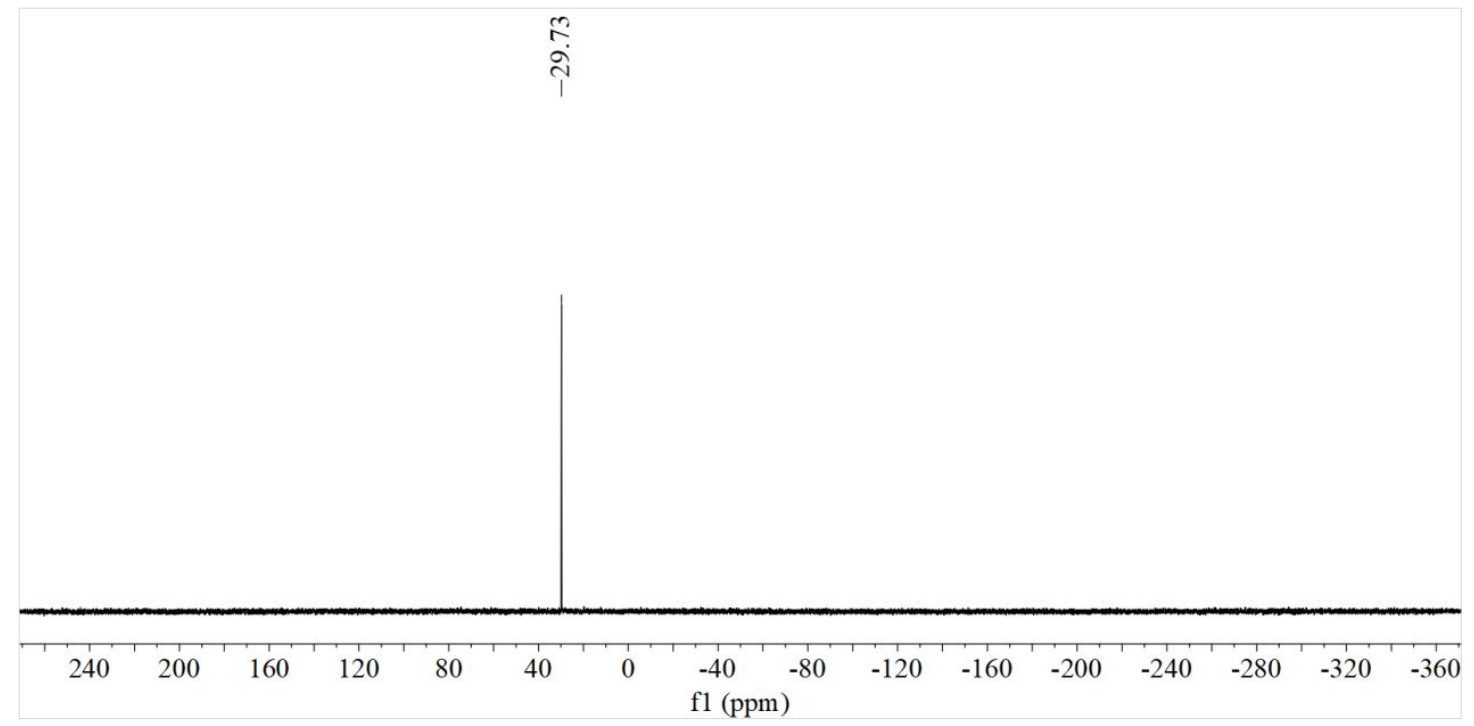

Figure S23. ${ }^{31} \mathrm{P}\left\{{ }^{1} \mathrm{H}\right\}$ NMR spectrum of $6\left(162 \mathrm{MHz}, \mathrm{CDCl}_{3}\right)$.

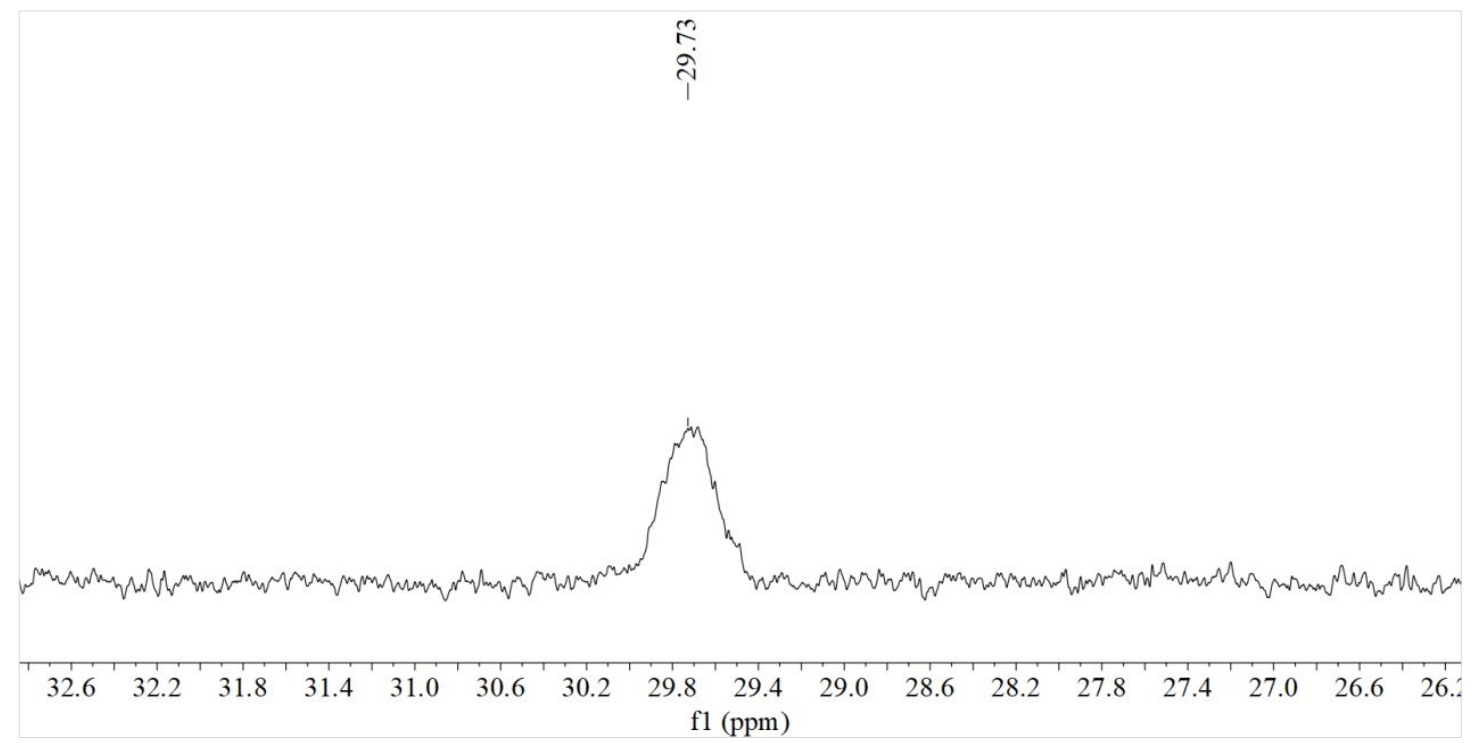

Figure S24. ${ }^{31} \mathrm{P}$ NMR spectrum of $7\left(162 \mathrm{MHz}, \mathrm{CDCl}_{3}\right)$. 


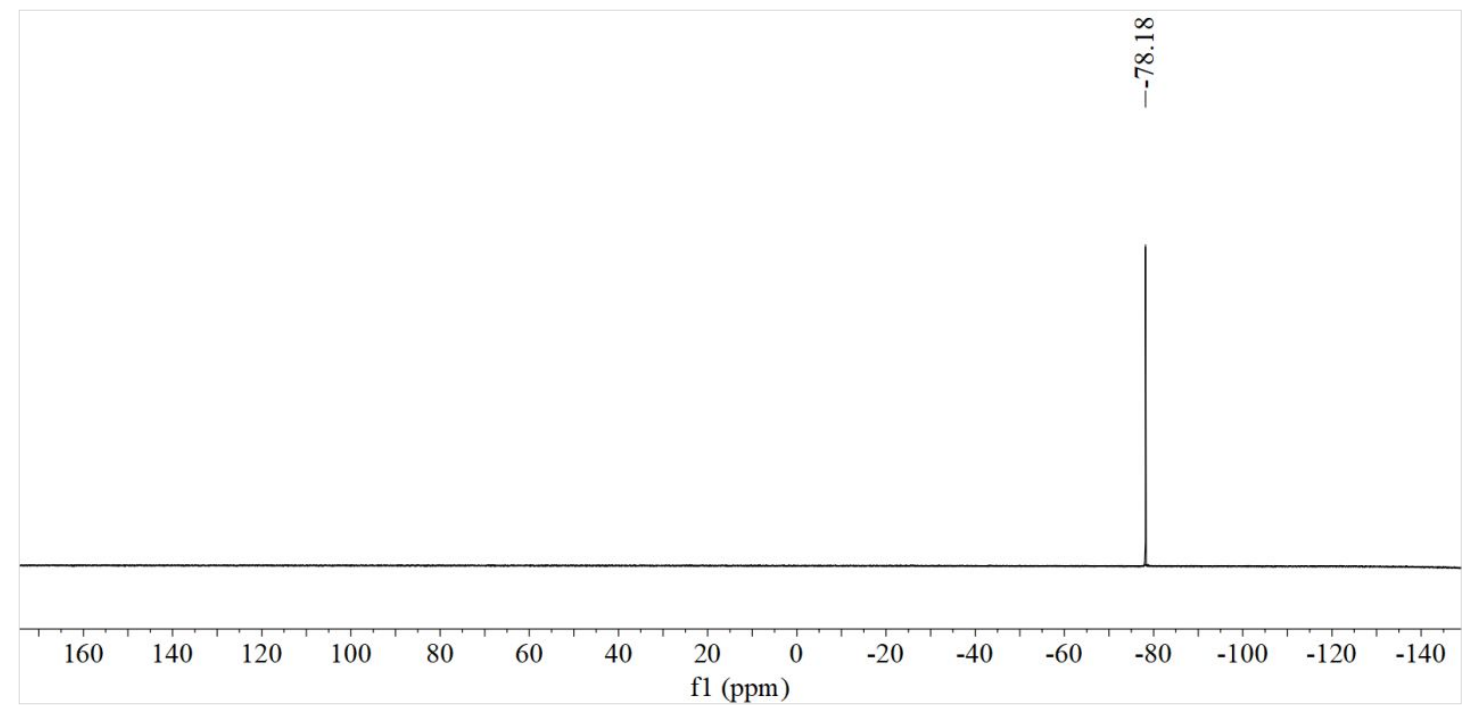

Figure S25. ${ }^{19} \mathrm{~F}\left\{{ }^{1} \mathrm{H}\right\}$ NMR spectrum of $7\left(377 \mathrm{MHz}, \mathrm{CDCl}_{3}\right)$. 


\section{Van't Hoff Analysis}

3 (15 mg, $0.0260 \mathrm{mmol})$ and excess of $\mathrm{IPr}(4.6 \mathrm{mg}, 0.030 \mathrm{mmol})$ were dissolved in $\mathrm{ClC}_{6} \mathrm{H}_{5}(0.8$ $\mathrm{mL}$ ) and the solution transferred into a Young's tap NMR tube. The formation of the previously characterized 4 was confirmed by ${ }^{31} \mathrm{P}$ NMR spectroscopy. The sample was left to equilibrate at $298 \mathrm{~K}$ for 6 hours. Variable temperature ${ }^{31} \mathrm{P}$ NMR analysis was performed on the sample over the temperature range $268-298 \mathrm{~K}$. At temperatures lower than $278 \mathrm{~K}$ it was found the be necessary to hold the sample at temperature for a prolonged period (about $30 \mathrm{~h}$ ) to allow equilibrium to be reached.

Table S1. Ratios of $\mathbf{4}$ and $\mathbf{5}$ over the temperature range $268-298 \mathrm{~K}$.

\begin{tabular}{|c|c|c|}
\hline Temperature & $\mathbf{4}$ & $\mathbf{5}$ \\
\hline 298 & $8.7 \%$ & $91.3 \%$ \\
\hline 288 & $7.6 \%$ & $92.4 \%$ \\
\hline 278 & $6.5 \%$ & $93.5 \%$ \\
\hline 268 & $5.2 \%$ & $94.8 \%$ \\
\hline
\end{tabular}

The Van't Hoff equation was used in order to determine $\Delta \mathrm{H}$ and $\Delta \mathrm{S}$ from the slope and the intercept of the plot of $\ln \left(\mathrm{K}_{\text {eq }}\right)$ versus $1 / \mathrm{T}$. $\Delta \mathrm{G}$ was calculated according to Gibb's Free Energy equation.

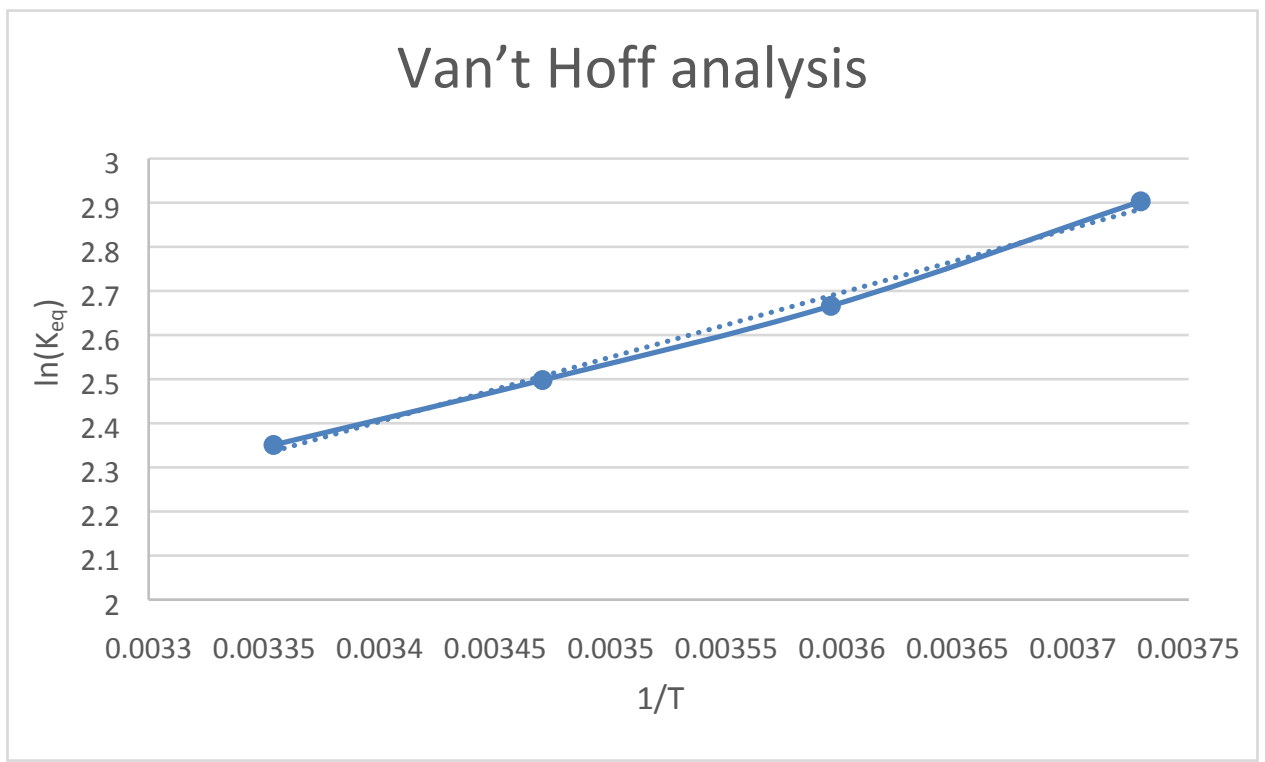

Figure S26. Van't Hoff Analysis

$\Delta \mathrm{H}=-2.9 \mathrm{kcal} / \mathrm{mol}$

$\Delta \mathrm{S}=-5.1 \mathrm{cal} / \mathrm{K} \mathrm{mol}$

$\Delta \mathrm{G}=-1.4 \mathrm{kcal} / \mathrm{mol}$ 


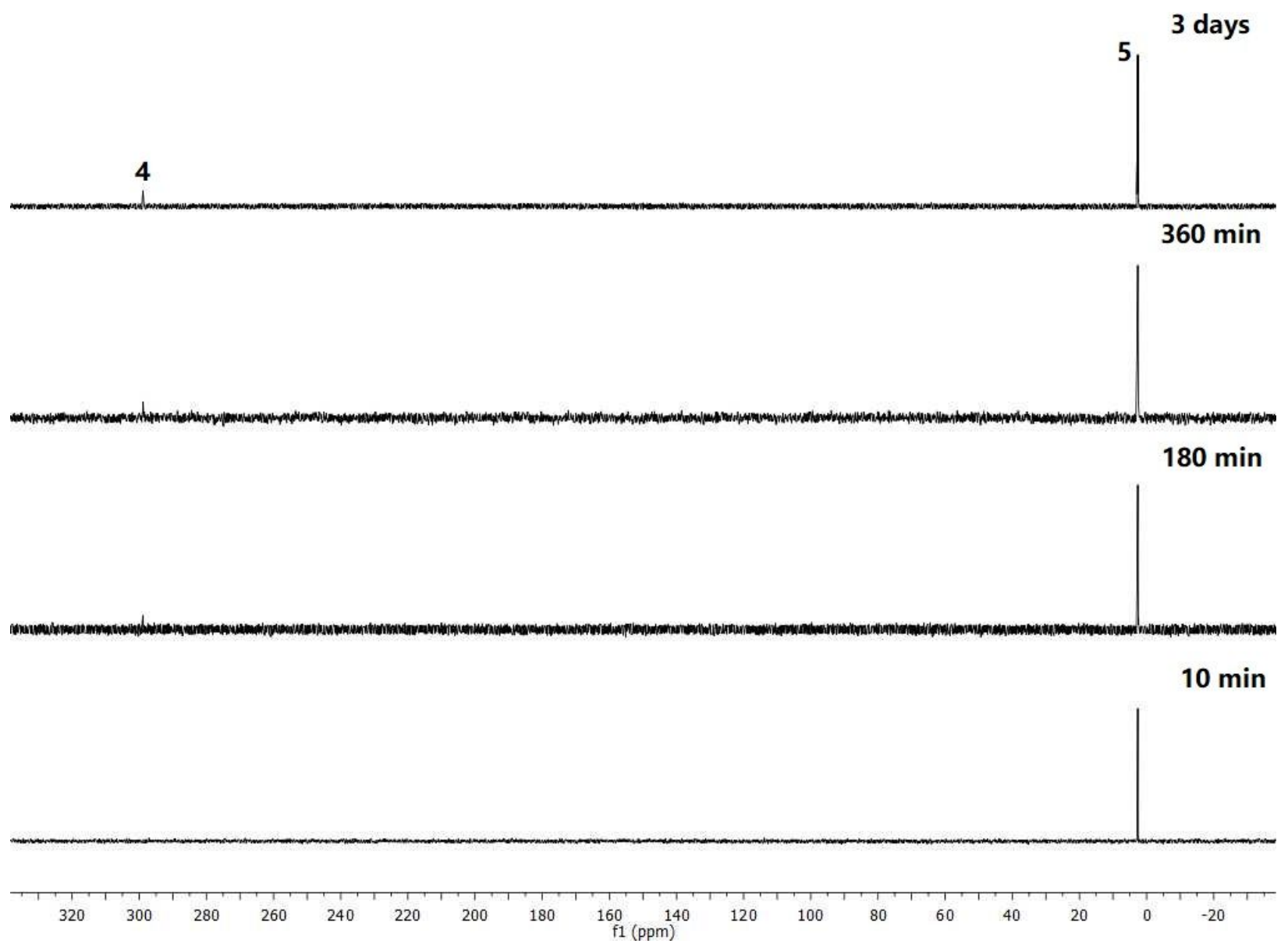

Figure S27. ${ }^{31} \mathrm{P}\left\{{ }^{1} \mathrm{H}\right\}$ NMR spectra for the establishment of an equilibrium between $\mathbf{4}$ and $\mathbf{5}$ upon dissolving the crystalline 5 in $\mathrm{ClC}_{6} \mathrm{H}_{5}$ at $25^{\circ} \mathrm{C}$. 


\section{Crystallographic Data}

X-ray Data Collection and Reduction. Crystals were coated in Paratone-N oil in an $\mathrm{N}_{2}$ filled glovebox, mounted on a MiTegen Micromount, and placed under a $\mathrm{N}_{2}$ stream, thus maintaining a dry, $\mathrm{O}_{2}$-free environment for each crystal. The data were collected on a Bruker Apex II diffractometer using a graphite monochromator with Mo Ka radiation $(\lambda=0.71073 \AA)$. The data were collected at $150(2) \mathrm{K}$ for all crystals. The frames were integrated with the Bruker SAINT software package using a narrow-frame algorithm. Data were corrected for absorption effects using the empirical multiscan method (SADABS).

Structure Solution and Refinement. The structures of 2, 5, 6 and 7 were solved by intrinsic phasing using XS. All the structures were subjected to full-matrix least-squares refinement on $\mathrm{F}^{2}$ using XL as implemented in the SHELXTL suite of programs. All non-hydrogen atoms were refined with anisotropically thermal parameters. Carbon bound hydrogen atoms were placed in geometrically calculated positions and refined using an appropriate riding model and coupled isotropic thermal parameters. 3 was modeled as a pentane with $50 \%$ occupancy disordered over an inversion center, whereas 7 was solved with a difluorobenzene molecule disordered over an inversion center with $50 \%$ occupancy. 
Table S2. Summary of crystallographic data for compounds 2, 5, 6 and 7.

\begin{tabular}{|c|c|c|c|c|}
\hline & 2 & 5 & 6 & 7 \\
\hline $\begin{array}{l}\text { empirical } \\
\text { formula }\end{array}$ & C34 H52 N P & C28 H46 N P & C36.50 H59 N P S & $\begin{array}{c}\text { C39 H57 F4 N O3 P } \\
\text { S }\end{array}$ \\
\hline $\begin{array}{l}\text { formula } \\
\text { weight }\end{array}$ & 505.73 & 427.63 & 574.87 & 726.88 \\
\hline crystal system & Monoclinic & Monoclinic & Monoclinic & Triclinic \\
\hline space group & $P 2_{1} / \mathrm{n}$ & $P 2_{1} / \mathrm{n}$ & $P 2_{1} / \mathrm{n}$ & $P \overline{1}$ \\
\hline$a(\AA)$ & $16.497(2)$ & $11.11(2)$ & $10.1440(5)$ & $11.096(7)$ \\
\hline$b(\AA)$ & $8.972(1)$ & $15.85(2)$ & $16.2799(7)$ & $11.353(9)$ \\
\hline$c(\AA)$ & $20.610(2)$ & $14.68(2)$ & $20.1295(7)$ & $16.05(1)$ \\
\hline$\alpha$ (deg.) & & & & $76.64(2)$ \\
\hline$\beta$ (deg.) & $105.159(4)$ & $95.94(5)$ & $104.252(2)$ & $69.87(2)$ \\
\hline$\gamma($ deg. $)$ & & & & $79.27(2)$ \\
\hline $\operatorname{vol}\left(\AA^{3}\right)$ & $2944.0(6)$ & $2569(7)$ & $3221.9(2)$ & $1834(2)$ \\
\hline$Z$ & 4 & 4 & 4 & 2 \\
\hline $\begin{array}{l}\rho(\text { calcd }) \\
\left(\mathrm{Mg} \cdot \mathrm{cm}^{3}\right)\end{array}$ & 1.141 & 1.106 & 1.750 & 1.316 \\
\hline$\mu\left(\mathrm{mm}^{-1}\right)$ & 0.116 & 0.121 & 0.176 & 0.191 \\
\hline$F(000)$ & 1112 & 944 & 1264 & 778 \\
\hline $\begin{array}{l}\text { Theta range } \\
\text { ( })\end{array}$ & 1.414 to 25.705 & 1.897 to 25.907 & 2.420 to 26.022 & 2.543 to 27.194 \\
\hline$T(\mathrm{~K})$ & $150(2)$ & $150(2)$ & $150(2)$ & $150(2)$ \\
\hline $\begin{array}{l}\text { reflections } \\
\text { collected }\end{array}$ & 46001 & 41636 & 46216 & 33504 \\
\hline $\begin{array}{l}\text { unique } \\
\text { reflections }\end{array}$ & 5588 & 4968 & 6340 & 8121 \\
\hline $\mathrm{R}_{\text {int }}$ & 0.1029 & 0.1223 & 0.1252 & 0.0493 \\
\hline GOF $\left(\mathrm{F}^{2}\right)$ & 1.075 & 1.079 & 1.032 & 1.054 \\
\hline $\begin{array}{l}\text { R1 indices } \\
{[\mathrm{I}>2 \sigma(\mathrm{I})]}\end{array}$ & 0.0515 & 0.0614 & 0.0615 & 0.0496 \\
\hline $\begin{array}{l}\text { wR2 indices } \\
\text { (all data) }\end{array}$ & 0.1269 & 0.1703 & 0.1705 & 0.1285 \\
\hline $\begin{array}{l}\text { Largest diff. } \\
\text { peak and hole } \\
\left(\text { e. } \AA^{-3}\right)\end{array}$ & $0.345 \&-0.322$ & $0.482 \&-0.635$ & $0.619 \&-0.683$ & $0.451 \&-0.449$ \\
\hline CCDC No. & 1907556 & 1907555 & 1907557 & 1907558 \\
\hline
\end{tabular}




\section{Computational Details}

Calculations were carried out with the Gaussian 09 package. ${ }^{\mathrm{S3}}$ Geometry optimizations were performed with the M06-2X functional. ${ }^{\mathrm{S}}$ The Def2-SVP basis set was used for all the atoms. Frequency calculations at the same level of theory were performed to identify the number of imaginary frequencies (zero for local minimum and one for transition states) and provide the thermal corrections of Gibbs free energy. Transition states were submitted to intrinsic reaction coordinate (IRC) calculations to determine two corresponding minima.

The single-point energy calculations were performed at the M06-2X/Def2-TZVP level of theory for solution-phase (benzene). The gas-phase geometry was used for all the solution phase calculations. The SMD method was used with the corresponding solvent, while Bondi radii ${ }^{55}$ were chosen as the atomic radii to define the molecular cavity. The Gibbs energy and enthalpy corrections from frequency calculations were added to the single-point energies to obtain the Gibbs free energies and enthalpies in solution, respectively. All the solution-phase free energies reported in the paper correspond to the reference state of $1 \mathrm{~mol} / \mathrm{L}, 298 \mathrm{~K}$. Natural localized molecular orbital (NLMO) calculations were carried out using NBO 6.0 program $^{\mathrm{S} 6}$ at the M062X/Def2-TZVP//M06-2X/Def2-SVP level of theory. Optimized structures were visualized by the CYLview, ${ }^{\mathrm{S7}}$ Chemcraft ${ }^{\mathrm{S} 8}$ or IBOview program. ${ }^{\mathrm{S} 9}$ Isotropic shifts for $\mathbf{4}$ and $\mathbf{5}$ were computed at the SMD(benzene)-GIAO-B97D/Def2-TZVP//M06-2X/Def2-SVP level of theory using experimentally observed chemical shift of $\mathbf{5}(7.6 \mathrm{ppm}$, benzene) as the reference. Intrinsic bond orbitals (IBOs) were carried out using IBOview program at the PBE/Def2-TZVP//M06-2X/Def2SVP level of theory. 


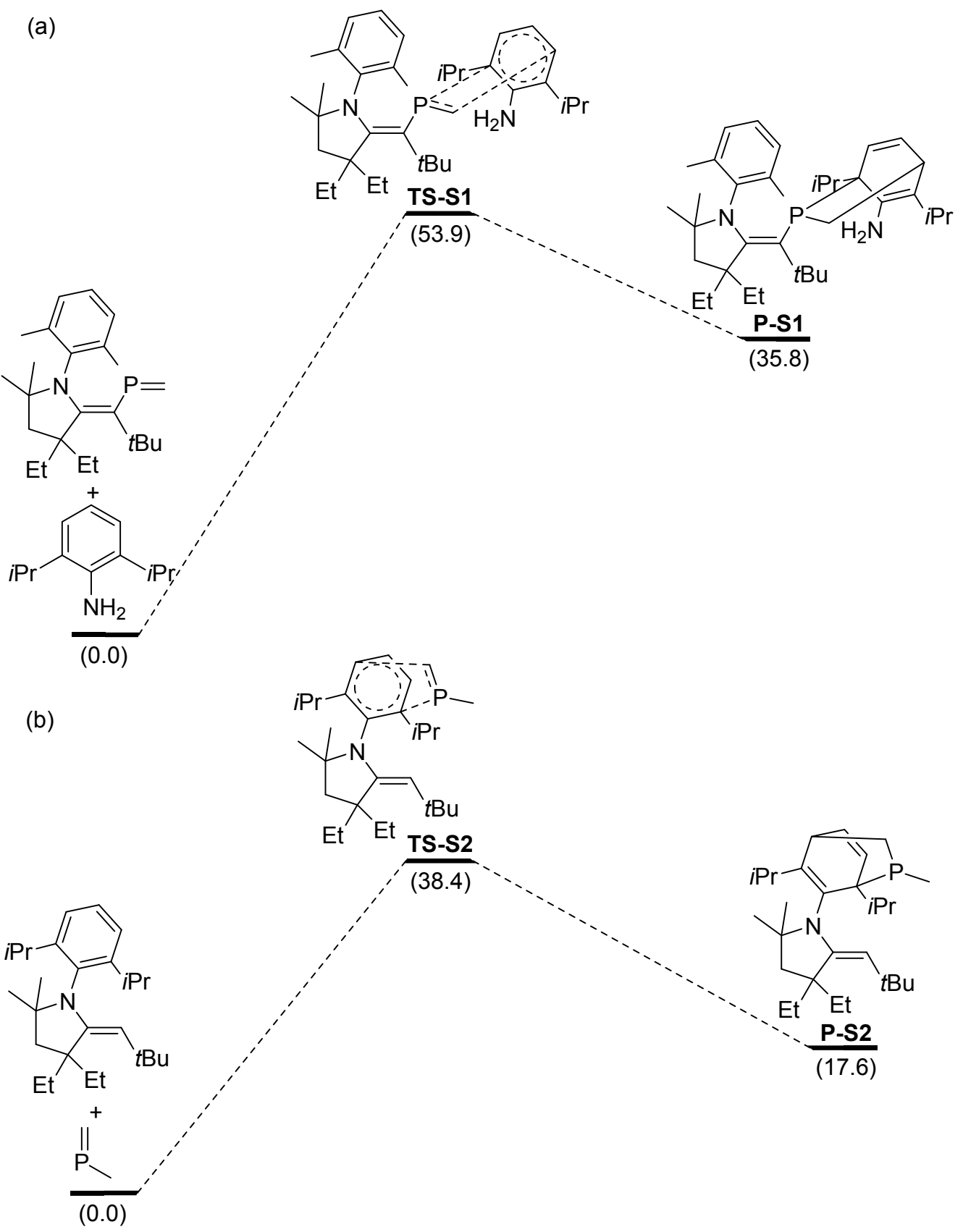

Figure S28. Free energy profile for intermolecular cycloadditions. Energies are given in $\mathrm{kcal} / \mathrm{mol}$. 


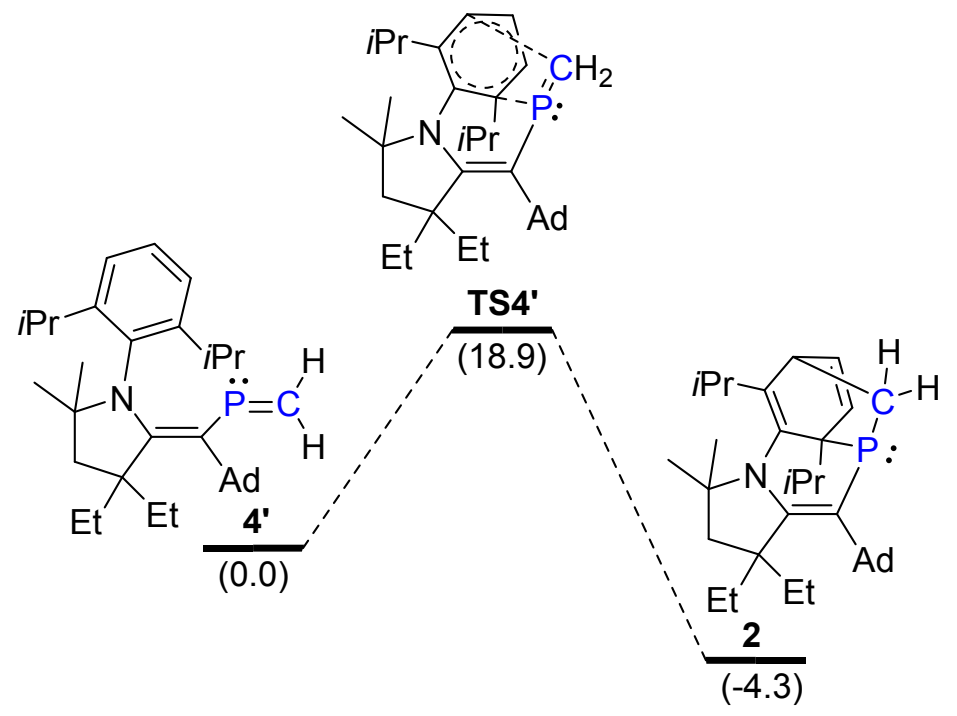

Figure S29. Free energy profile for the irreversible cycloaddition of 4' leading to 2. Energies are given in $\mathrm{kcal} / \mathrm{mol}$.

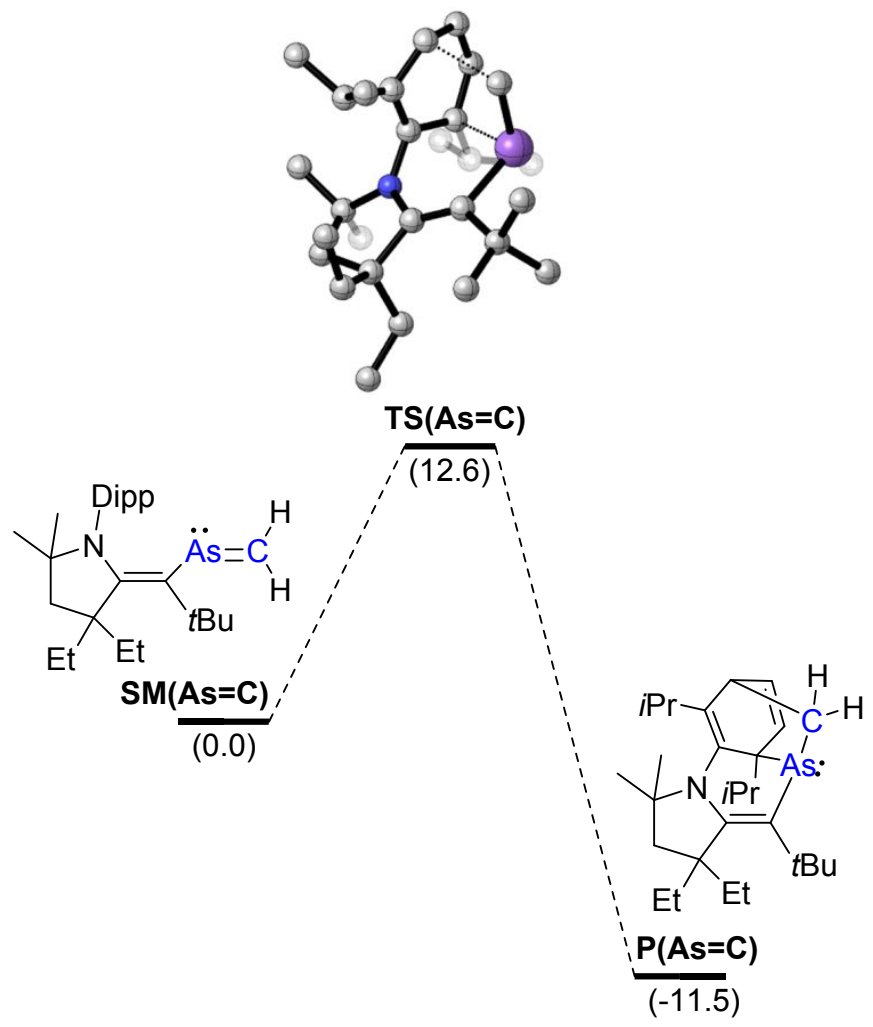

Figure S30. Free energy profile for the cycloaddition involving $\mathrm{As}=\mathrm{C}$ double bond. Energies are given in $\mathrm{kcal} / \mathrm{mol}$. 


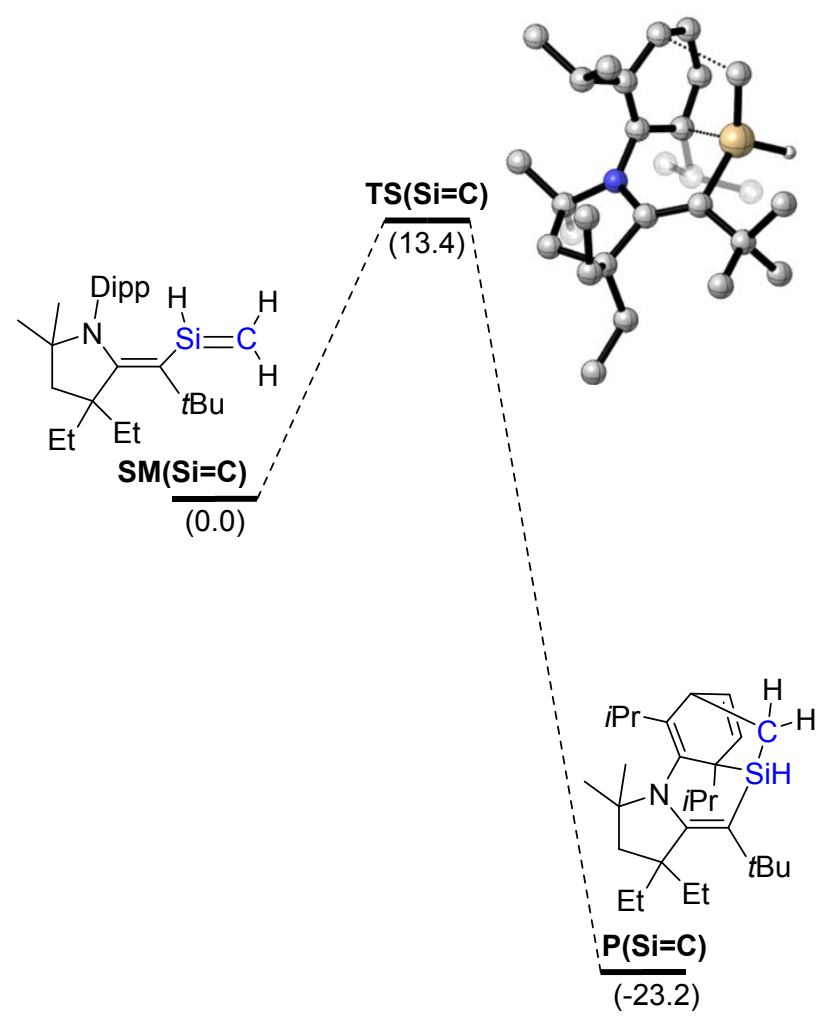

Figure S31. Free energy profile for the cycloaddition involving $\mathrm{Si}=\mathrm{C}$ double bond. Energies are given in $\mathrm{kcal} / \mathrm{mol}$.

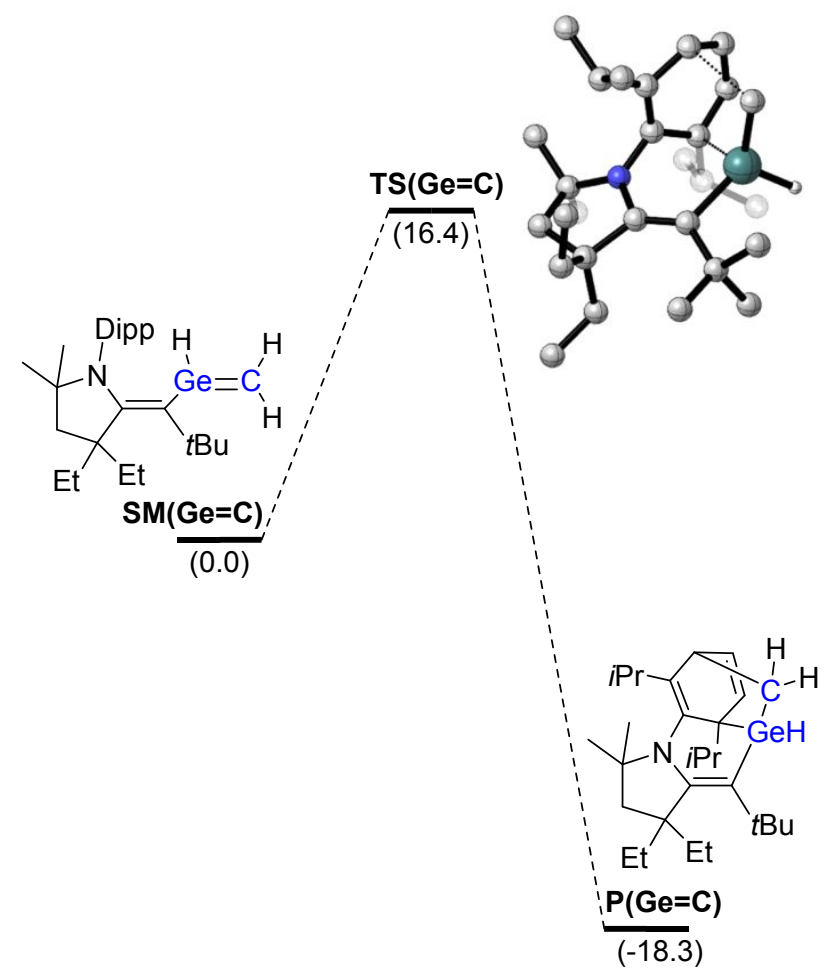

Figure S32. Free energy profile for the cycloaddition involving $\mathrm{Ge}=\mathrm{C}$ double bond. Energies are given in $\mathrm{kcal} / \mathrm{mol}$. 


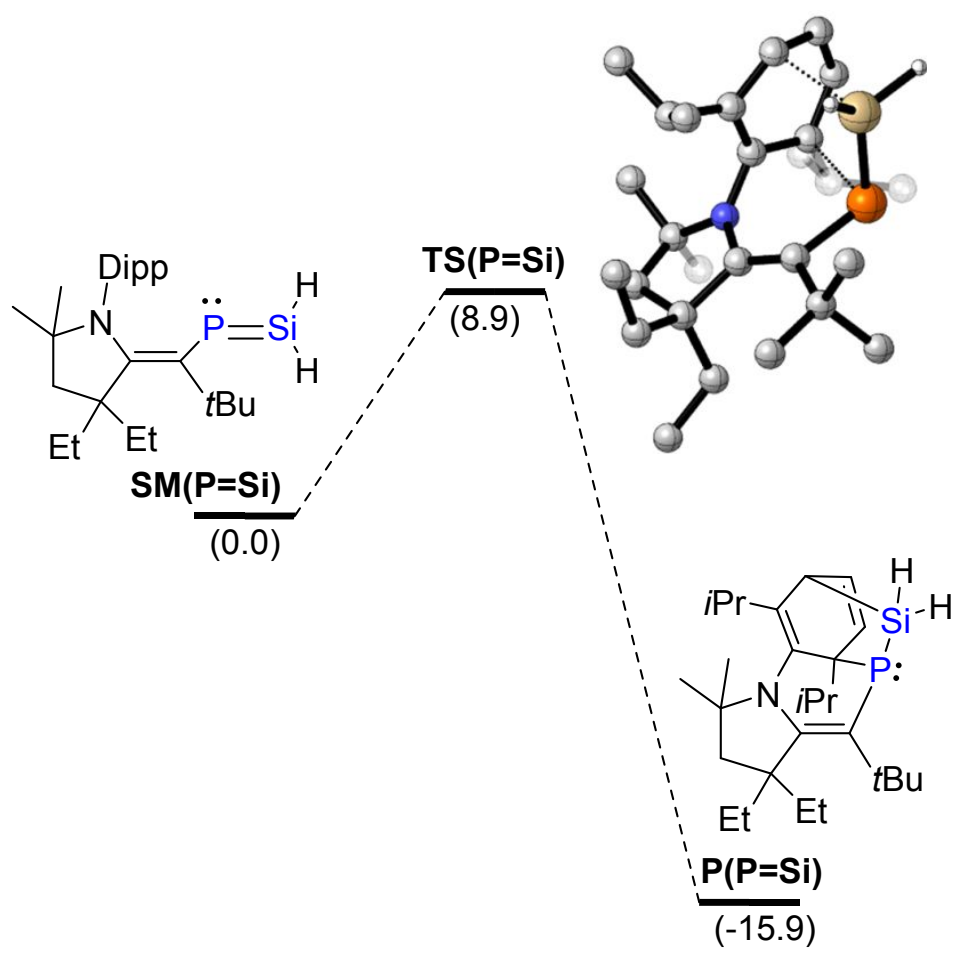

Figure S33. Free energy profile for the cycloaddition involving $\mathrm{P}=\mathrm{Si}$ double bond. Energies are given in $\mathrm{kcal} / \mathrm{mol}$.

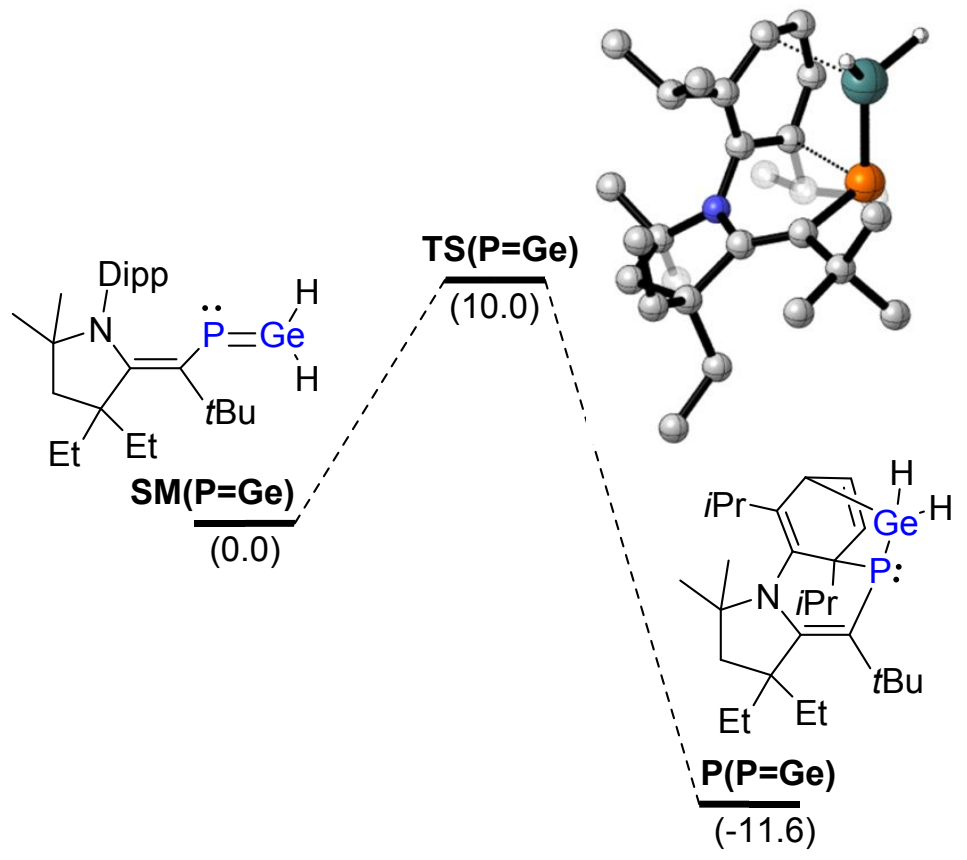

Figure S34. Free energy profile for the cycloaddition involving $\mathrm{P}=\mathrm{Ge}$ double bond. Energies are given in $\mathrm{kcal} / \mathrm{mol}$. 
Table S3. Energy of Intermediates and Transition States.

\begin{tabular}{|c|c|c|}
\hline Species & $\begin{array}{l}\text { Thermal Corrections of } \\
\text { Gibbs Free Energies } \\
\text { (Enthalpies are given in } \\
\text { parentheses) (Hartree) }\end{array}$ & Solvation Energies (Hartree) \\
\hline $\mathrm{C}_{6} \mathrm{H}_{6}$ & $0.074005(0.106759)$ & -232.231799 \\
\hline $\mathrm{HP}=\mathrm{CH}_{2}$ & $0.01036(0.037867)$ & -381.1984782 \\
\hline $\mathrm{H}_{2} \mathrm{C}=\mathrm{CH}_{2}$ & $0.028757(0.054948)$ & -78.57276254 \\
\hline & $0.106247(0.145054)$ & -613.4004361 \\
\hline & $0.110314(0.147879)$ & -613.439656 \\
\hline & $0.125245(0.162525)$ & -310.7478732 \\
\hline & $0.129951(0.166072)$ & -310.7997001 \\
\hline 3 & 0.656418 & -2452.804153 \\
\hline KHMDS & 0.178805 & -1473.240711 \\
\hline $\mathrm{K}\left(\mathrm{C}_{6} \mathrm{H}_{6}\right) \mathrm{OTf}$ & 0.082802 & -1793.803911 \\
\hline TS1 & 0.840314 & -2364.463789 \\
\hline HMDS & 0.195227 & -873.8993223 \\
\hline IN1 & 0.623882 & -1490.57593 \\
\hline TS2 & 0.623828 & -1490.555371 \\
\hline IN2 & 0.62415 & -1490.56774 \\
\hline TS3 & 0.624239 & -1490.563003 \\
\hline 4 & 0.625942 & -1490.611002 \\
\hline TS4 & 0.627768 & -1490.58 \\
\hline 5 & 0.631913 & -1490.62184 \\
\hline 4 ' & 0.736758 & -1722.869536 \\
\hline TS4' & 0.738059 & -1722.840758 \\
\hline 2 & 0.740929 & -1722.880567 \\
\hline & 0.516412 & -1333.388199 \\
\hline & 0.248796 & -523.4433615 \\
\hline TS-S1 & 0.791384 & -1856.771867 \\
\hline P-S1 & 0.795079 & -1856.804448 \\
\hline
\end{tabular}




\begin{tabular}{|c|c|c|}
\hline & 0.6093 & -1110.620616 \\
\hline $\mathbf{T S}-\mathbf{S 2}$ & & \\
\hline $\mathbf{P}-\mathbf{S 2}$ & 0.036308 & -420.5149124 \\
\hline $\mathbf{S M}(\mathbf{A s}=\mathbf{C})$ & 0.669985 & -1531.098697 \\
\hline $\mathbf{T S}(\mathbf{A s}=\mathbf{C})$ & 0.67437 & -1531.136289 \\
\hline $\mathbf{P}(\mathbf{A s}=\mathbf{C})$ & 0.630108 & -3385.146831 \\
\hline $\mathbf{S M}(\mathbf{S i}=\mathbf{C})$ & 0.626023 & -3385.122687 \\
\hline $\mathbf{T S}(\mathbf{S i}=\mathbf{C})$ & 0.630108 & -3385.16517 \\
\hline $\mathbf{P}(\mathbf{S i}=\mathbf{C})$ & 0.632543 & -1439.335782 \\
\hline $\mathbf{S M}(\mathbf{G e}=\mathbf{C})$ & 0.63679 & -1439.318753 \\
\hline $\mathbf{T S}(\mathbf{G e}=\mathbf{C})$ & 0.639889 & -1439.380111 \\
\hline $\mathbf{P}(\mathbf{G e}=\mathbf{C})$ & 0.629665 & -3226.856285 \\
\hline $\mathbf{S M}(\mathbf{P}=\mathbf{S i})$ & 0.633961 & -3226.834527 \\
\hline $\mathbf{T S}(\mathbf{P}=\mathbf{S i})$ & 0.63673 & -3226.892532 \\
\hline $\mathbf{P}(\mathbf{P}=\mathbf{S i})$ & 0.615824 & -1741.980019 \\
\hline $\mathbf{S M}(\mathbf{P}=\mathbf{G e})$ & 0.617612 & -1741.967571 \\
\hline $\mathbf{T S}(\mathbf{P}=\mathbf{G e})$ & 0.622461 & -1742.011966 \\
\hline $\mathbf{P}(\mathbf{P}=\mathbf{G e})$ & 0.61393 & -3529.511532 \\
\hline & 0.615736 & -3529.497454 \\
\hline & 0.620294 & -3529.536452 \\
\hline & & \\
\hline & & \\
\hline & & \\
\hline & & \\
\hline
\end{tabular}

\section{Cartesian Coordinates:}

$\mathrm{C}_{6} \mathrm{H}_{6}$ :

$\begin{array}{lrrr}6 & -0.032647 & 0.721432 & 0.000069 \\ 6 & 1.362168 & 0.721404 & 0.000634 \\ 6 & 2.059276 & 1.929218 & -0.000108 \\ 6 & 1.362201 & 3.137278 & -0.001004 \\ 6 & -0.032409 & 3.137309 & -0.001398 \\ 6 & -0.729625 & 1.929333 & -0.001010 \\ 1 & -0.578482 & -0.223008 & 0.000438 \\ 1 & 1.907877 & -0.223116 & 0.001372 \\ 1 & 3.150013 & 1.929313 & 0.000146 \\ 1 & 1.908267 & 4.081576 & -0.001466 \\ 1 & -0.578330 & 4.081696 & -0.002230 \\ 1 & -1.820370 & 1.929498 & -0.001353\end{array}$
$\mathrm{HP}=\mathrm{CH}_{2}$ :
$\begin{array}{lllll}15 & 0.647279 & -1.832015 & 10.870176\end{array}$
$\begin{array}{lllll}6 & 1.181946 & -1.999976 & 9.305983\end{array}$
$\begin{array}{lllll}1 & 1.999857 & -1.365780 & 8.950706\end{array}$
$\begin{array}{llll}1 & 0.779659 & -2.708049 & 8.576938\end{array}$
$\begin{array}{llll}1 & -0.373089 & -2.832482 & 10.831121\end{array}$ 


$$
\begin{aligned}
& \mathrm{H}_{2} \mathrm{C}=\mathrm{CH}_{2} \text { : } \\
& \begin{array}{llll}
6 & 1.043610 & -2.004041 & 9.348232
\end{array} \\
& \begin{array}{lllll}
1 & 1.897652 & -1.436137 & 8.971733
\end{array} \\
& \begin{array}{lllll}
1 & 0.539908 & -2.666036 & 8.639993
\end{array} \\
& \begin{array}{lllll}
1 & -0.216492 & -2.463324 & 10.985700
\end{array} \\
& \begin{array}{lllll}
6 & 0.637333 & -1.895164 & 10.609108
\end{array} \\
& \begin{array}{lllll}
1 & 1.141596 & -1.233661 & 11.317407
\end{array} \\
& {\left[\begin{array}{l}
1 \\
1 \\
1 \\
\hdashline
\end{array}\right.} \\
& \begin{array}{lllll}
15 & 0.676737 & -1.890716 & 10.918184
\end{array} \\
& \begin{array}{llll}
6 & -1.806646 & -0.784955 & 9.798233
\end{array} \\
& \begin{array}{lllll}
1 & -2.720570 & -1.259640 & 10.159552
\end{array} \\
& \begin{array}{lllll}
6 & -0.958947 & -0.086896 & 10.714575
\end{array} \\
& \begin{array}{lllll}
6 & 1.106559 & -1.894674 & 9.238204
\end{array} \\
& \begin{array}{lllll}
1 & 2.067494 & -1.443074 & 8.980217
\end{array} \\
& \begin{array}{lllll}
1 & 0.819556 & -2.734118 & 8.601094
\end{array} \\
& \begin{array}{lllll}
6 & 0.376057 & 0.724097 & 8.898468
\end{array} \\
& \begin{array}{llll}
6 & -0.086042 & -0.429859 & 8.179763
\end{array} \\
& \begin{array}{lllll}
1 & 0.288170 & -0.584969 & 7.165641
\end{array} \\
& \begin{array}{lllll}
6 & -0.058768 & 0.892048 & 10.178253
\end{array} \\
& \begin{array}{llll}
6 & -1.378221 & -0.941384 & 8.510354
\end{array} \\
& \begin{array}{lllll}
1 & -1.929749 & -1.551369 & 7.793500
\end{array} \\
& \begin{array}{lllll}
1 & 0.338270 & 1.677149 & 10.823244
\end{array} \\
& \begin{array}{lllll}
1 & 1.137832 & 1.372036 & 8.461860
\end{array} \\
& \begin{array}{llll}
1 & -1.287508 & 0.021992 & 11.750010
\end{array} \\
& \begin{array}{llll}
1 & -0.361194 & -2.866021 & 10.853925
\end{array}
\end{aligned}
$$




$$
\begin{array}{llll}
1 & -1.193215 & -0.128620 & 11.755617 \\
1 & -0.308656 & -2.795586 & 10.940934
\end{array}
$$<smiles>c1cc2ccc1cc2</smiles>

$\begin{array}{cccc}6 & -1.839137 & -0.780393 & 9.731369 \\ 1 & -2.773163 & -1.220514 & 10.084734 \\ 6 & -0.911984 & -0.198810 & 10.661272 \\ 6 & 0.947476 & -1.876504 & 9.268016 \\ 1 & 1.938553 & -1.509000 & 8.999611 \\ 1 & 0.601323 & -2.722142 & 8.672665 \\ 6 & 0.364100 & 0.725473 & 8.875135 \\ 6 & -0.100267 & -0.420276 & 8.143839 \\ 1 & 0.281043 & -0.568717 & 7.130727 \\ 6 & -0.050975 & 0.838324 & 10.165821 \\ 6 & -1.423512 & -0.893738 & 8.440963 \\ 1 & -2.000631 & -1.432080 & 7.687376 \\ 1 & 0.343595 & 1.599253 & 10.841180 \\ 1 & 1.114291 & 1.390602 & 8.444243 \\ 1 & -1.196477 & -0.167167 & 11.715766 \\ 1 & -0.151742 & -2.519903 & 11.003557 \\ 6 & 0.519731 & -1.762359 & 10.597948 \\ 1 & 1.188266 & -1.308454 & 11.330375\end{array}$

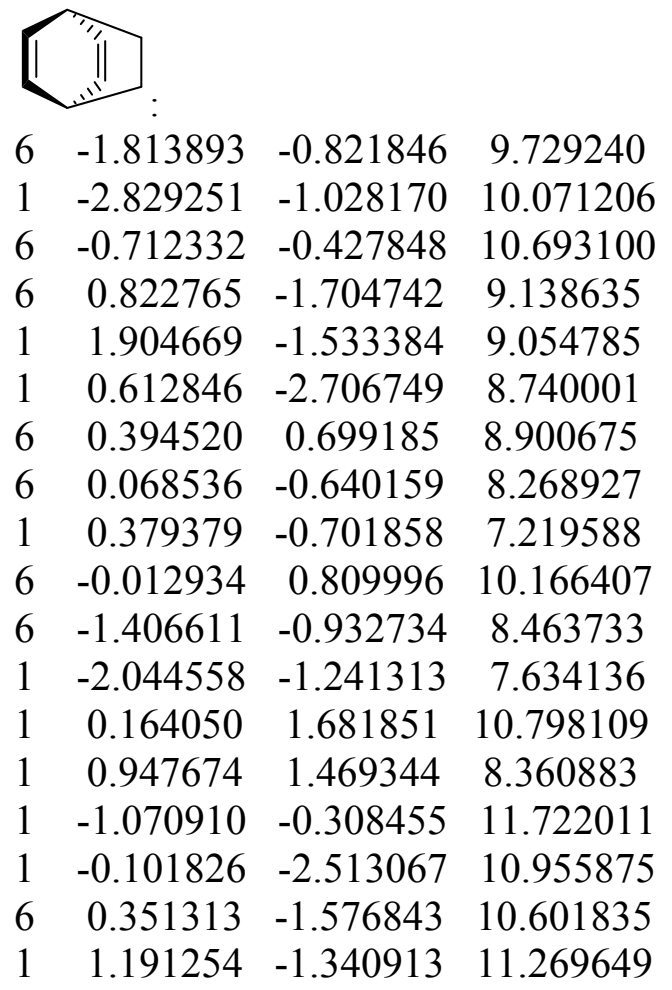




\begin{tabular}{cccc} 
3: & & & \\
15 & 8.244789 & 3.705950 & 4.686105 \\
7 & 8.461612 & 5.915835 & 3.322943 \\
6 & 8.813548 & 5.184638 & 5.575798 \\
6 & 8.812268 & 6.203774 & 4.661469 \\
6 & 9.078040 & 7.712394 & 4.738370 \\
6 & 8.363708 & 8.233214 & 3.460946 \\
1 & 9.097909 & 8.637387 & 2.749296 \\
1 & 7.655266 & 9.040928 & 3.691061 \\
6 & 7.627068 & 7.053026 & 2.818315 \\
6 & 7.568264 & 7.226259 & 1.309881 \\
1 & 7.010739 & 8.151459 & 1.103249 \\
1 & 7.035800 & 6.402028 & 0.820733 \\
1 & 8.568368 & 7.334240 & 0.869353 \\
6 & 6.205624 & 6.897944 & 3.367619 \\
1 & 5.574473 & 7.734364 & 3.035671 \\
1 & 6.206029 & 6.881752 & 4.466288 \\
1 & 5.755117 & 5.957521 & 3.013586 \\
6 & 10.597021 & 8.004001 & 4.622513 \\
1 & 11.074709 & 7.881351 & 5.603825 \\
1 & 10.697451 & 9.070305 & 4.364379 \\
6 & 11.338893 & 7.162757 & 3.589903 \\
1 & 12.388727 & 7.478622 & 3.521364 \\
1 & 10.891599 & 7.255477 & 2.588364 \\
1 & 11.323875 & 6.095139 & 3.857882 \\
6 & 8.469833 & 8.387899 & 5.980932 \\
1 & 8.847192 & 7.908780 & 6.888875 \\
1 & 7.381176 & 8.210046 & 5.970650 \\
6 & 8.746638 & 9.885818 & 6.080496 \\
1 & 8.186545 & 10.320225 & 6.919498 \\
1 & 8.452068 & 10.428079 & 5.169740 \\
1 & 9.812031 & 10.087116 & 6.259117 \\
6 & 8.176513 & 4.565561 & 3.099847 \\
6 & 8.079363 & 3.873762 & 1.921930 \\
6 & 7.489199 & 2.555792 & 1.867300 \\
1 & 7.686581 & 2.008477 & 0.941939 \\
6 & 6.622283 & 1.932726 & 2.718498 \\
1 & 6.228920 & 0.979877 & 2.353500 \\
6 & 6.065685 & 2.321283 & 3.993796 \\
1 & 5.118828 & 1.826939 & 4.227046 \\
6 & 6.576009 & 3.092258 & 4.985712 \\
6 & 8.725375 & 4.363089 & 0.632188 \\
1 & 9.044695 & 5.396223 & 0.795363 \\
6 & 10.005707 & 3.545575 & 0.402966 \\
1 & 10.699104 & 3.651728 & 1.249281 \\
1 & 10.516222 & 3.891721 & -0.507876 \\
& & & \\
\hline
\end{tabular}




$\begin{array}{cccc}1 & 9.784535 & 2.475338 & 0.275523 \\ 6 & 7.818664 & 4.296738 & -0.598862 \\ 1 & 8.297141 & 4.800245 & -1.451640 \\ 1 & 6.840569 & 4.766687 & -0.425710 \\ 1 & 7.632352 & 3.254370 & -0.896753 \\ 6 & 5.858767 & 3.390016 & 6.286972 \\ 1 & 6.615467 & 3.286903 & 7.079317 \\ 6 & 5.299211 & 4.817156 & 6.304108 \\ 1 & 4.819091 & 5.019696 & 7.273037 \\ 1 & 4.544602 & 4.941310 & 5.511927 \\ 1 & 6.086170 & 5.570039 & 6.155365 \\ 6 & 4.764395 & 2.372705 & 6.599845 \\ 1 & 4.381318 & 2.556013 & 7.613585 \\ 1 & 5.177794 & 1.356766 & 6.564749 \\ 1 & 3.916432 & 2.462217 & 5.902722 \\ 6 & 9.480243 & 2.389353 & 4.541520 \\ 1 & 9.545961 & 1.812755 & 5.473075 \\ 1 & 10.431668 & 2.883288 & 4.299495 \\ 1 & 9.177113 & 1.723654 & 3.724910 \\ 6 & 9.272134 & 5.117058 & 7.047178 \\ 6 & 8.184684 & 5.585034 & 8.035132 \\ 1 & 7.382914 & 4.839732 & 8.117766 \\ 1 & 7.729631 & 6.543403 & 7.759391 \\ 6 & 9.594410 & 3.660224 & 7.406793 \\ 1 & 10.484729 & 3.286648 & 6.882145 \\ 1 & 8.764364 & 2.972672 & 7.193763 \\ 6 & 10.573251 & 5.904290 & 7.280533 \\ 1 & 10.431346 & 6.989841 & 7.319835 \\ 1 & 11.316532 & 5.674080 & 6.502517 \\ 16 & 8.289280 & 0.207495 & 7.374672 \\ 9 & 7.492920 & 2.085822 & 9.061448 \\ 9 & 8.047999 & 0.233736 & 10.011021 \\ 9 & 6.184170 & 0.384571 & 8.944897 \\ 8 & 9.674391 & 0.667420 & 7.532482 \\ 8 & 8.063191 & -1.226202 & 7.349213 \\ 8 & 7.535962 & 0.996029 & 6.371105 \\ 6 & 7.463441 & 0.747929 & 8.940287 \\ 1 & 10.994561 & 5.599568 & 8.249470 \\ 1 & 8.631409 & 5.687194 & 9.035794 \\ 1 & 9.790661 & 3.573589 & 8.484323 \\ & & & \\ & & & \end{array}$

KHMDS:

$\begin{array}{llll}7 & -0.726883 & 0.258433 & 0.000519\end{array}$

$\begin{array}{lllll}14 & -1.590004 & 1.678098 & 0.286898\end{array}$

$\begin{array}{lllll}14 & 0.892724 & -0.161481 & -0.209207\end{array}$

$\begin{array}{llll}6 & 0.978640 & -2.067180 & -0.441957\end{array}$ 


$\begin{array}{cccc}1 & 0.402816 & -2.392991 & -1.328120 \\ 1 & 2.011523 & -2.413860 & -0.599843 \\ 1 & 0.603914 & -2.604099 & 0.449077 \\ 6 & 2.023309 & 0.225151 & 1.263373 \\ 1 & 2.097093 & 1.313899 & 1.413121 \\ 1 & 1.616607 & -0.206999 & 2.191031 \\ 1 & 3.043177 & -0.164606 & 1.117374 \\ 6 & 1.724161 & 0.576215 & -1.745155 \\ 1 & 2.751253 & 0.202128 & -1.880912 \\ 1 & 1.146444 & 0.339955 & -2.652416 \\ 1 & 1.772471 & 1.673424 & -1.662262 \\ 6 & -1.510748 & 2.969471 & -1.098710 \\ 1 & -0.482973 & 3.348977 & -1.211132 \\ 1 & -1.805110 & 2.526518 & -2.062935 \\ 1 & -2.167415 & 3.831023 & -0.898772 \\ 6 & -1.153597 & 2.578666 & 1.897734 \\ 1 & -1.820378 & 3.434516 & 2.088118 \\ 1 & -1.214622 & 1.893196 & 2.757394 \\ 1 & -0.120824 & 2.959172 & 1.853564 \\ 6 & -3.448609 & 1.211275 & 0.443063 \\ 1 & -4.075173 & 2.094545 & 0.641309 \\ 1 & -3.833504 & 0.760531 & -0.490593 \\ 1 & -3.625368 & 0.511850 & 1.281326 \\ 19 & -2.234807 & -1.727668 & -0.065521\end{array}$

$\begin{array}{cccc}\mathrm{K}\left(\mathrm{C}_{6} \mathrm{H}_{6}\right) \mathrm{OTf}: & & \\ 6 & 2.215500 & 2.439732 & 0.886941 \\ 16 & 0.451570 & 1.969071 & 1.096435 \\ 8 & -0.193219 & 2.251674 & -0.197805 \\ 8 & -0.061002 & 2.805979 & 2.191321 \\ 8 & 0.463543 & 0.532622 & 1.423338 \\ 9 & 2.312741 & 3.719177 & 0.564137 \\ 9 & 2.767503 & 1.713638 & -0.072293 \\ 9 & 2.877168 & 2.231651 & 2.014238 \\ 19 & -2.251542 & 1.134678 & 1.309559 \\ 6 & -5.231671 & 0.509600 & 2.420292 \\ 6 & -4.804972 & -0.746011 & 1.977870 \\ 6 & -4.602581 & -0.974599 & 0.613633 \\ 6 & -4.826900 & 0.052312 & -0.307762 \\ 6 & -5.252832 & 1.308116 & 0.134636 \\ 6 & -5.455616 & 1.536696 & 1.498742 \\ 1 & -5.398303 & 0.685572 & 3.484339 \\ 1 & -4.638133 & -1.549596 & 2.697039 \\ 1 & -4.274487 & -1.955993 & 0.267339 \\ 1 & -4.672439 & -0.126918 & -1.372955 \\ 1 & -5.430495 & 2.107799 & -0.586181\end{array}$




\begin{tabular}{lccc} 
TS1: & & & \\
15 & 8.275073 & 3.843018 & 4.301565 \\
7 & 8.519905 & 6.250436 & 3.320445 \\
6 & 8.775600 & 5.175190 & 5.443616 \\
6 & 8.816379 & 6.325083 & 4.705841 \\
6 & 9.075437 & 7.807727 & 5.017007 \\
6 & 8.359750 & 8.508106 & 3.830620 \\
1 & 9.090074 & 9.030078 & 3.195643 \\
1 & 7.633532 & 9.257645 & 4.175111 \\
6 & 7.652784 & 7.428136 & 3.004803 \\
6 & 7.584594 & 7.859143 & 1.549059 \\
1 & 7.011401 & 8.797275 & 1.509512 \\
1 & 7.065005 & 7.125972 & 0.922484 \\
1 & 8.582386 & 8.057381 & 1.135403 \\
6 & 6.235388 & 7.135854 & 3.511378 \\
1 & 5.586670 & 8.013481 & 3.378851 \\
1 & 6.240290 & 6.863175 & 4.578846 \\
1 & 5.802543 & 6.295089 & 2.946910 \\
6 & 10.596102 & 8.109274 & 4.951195 \\
1 & 11.068343 & 7.830547 & 5.903804 \\
1 & 10.702024 & 9.202551 & 4.865442 \\
6 & 11.346841 & 7.440713 & 3.804697 \\
1 & 12.393426 & 7.774535 & 3.789733 \\
1 & 10.900353 & 7.678909 & 2.827662 \\
1 & 11.341900 & 6.345233 & 3.905977 \\
6 & 8.464089 & 8.318800 & 6.335656 \\
1 & 8.821050 & 7.727404 & 7.183683 \\
1 & 7.373369 & 8.160937 & 6.290509 \\
6 & 8.755158 & 9.789482 & 6.628549 \\
1 & 8.186135 & 10.120554 & 7.507941 \\
1 & 8.480089 & 10.444910 & 5.788989 \\
1 & 9.819725 & 9.954411 & 6.845039 \\
6 & 8.279237 & 4.943150 & 2.875556 \\
6 & 8.180150 & 4.428813 & 1.610882 \\
6 & 7.625665 & 3.112992 & 1.379547 \\
1 & 7.890700 & 2.667589 & 0.416781 \\
6 & 6.714862 & 2.396331 & 2.102682 \\
1 & 6.358342 & 1.472525 & 1.640747 \\
6 & 6.049301 & 2.733955 & 3.341029 \\
1 & 5.009126 & 2.391008 & 3.393464 \\
6 & 6.518946 & 3.403080 & 4.425025 \\
6 & 8.728372 & 5.144974 & 0.383903 \\
1 & 9.030390 & 6.147710 & 0.700334 \\
6 & 10.006103 & 4.442364 & -0.095109 \\
& & & \\
\hline
\end{tabular}




$\begin{array}{cccc}1 & 10.747664 & 4.367729 & 0.712830 \\ 1 & 10.452834 & 5.009529 & -0.925486 \\ 1 & 9.801365 & 3.425126 & -0.459315 \\ 6 & 7.721121 & 5.249532 & -0.764817 \\ 1 & 8.101732 & 5.918687 & -1.550737 \\ 1 & 6.742550 & 5.624503 & -0.433283 \\ 1 & 7.551686 & 4.263682 & -1.222600 \\ 6 & 5.624874 & 3.856666 & 5.567748 \\ 1 & 6.101414 & 4.750301 & 6.004215 \\ 6 & 4.229646 & 4.271003 & 5.093204 \\ 1 & 3.670476 & 4.726432 & 5.923295 \\ 1 & 3.650227 & 3.401740 & 4.748865 \\ 1 & 4.282362 & 4.996190 & 4.269590 \\ 6 & 5.505309 & 2.791649 & 6.665491 \\ 1 & 4.859904 & 3.157009 & 7.478360 \\ 1 & 6.477013 & 2.517788 & 7.097248 \\ 1 & 5.062494 & 1.871361 & 6.254401 \\ 6 & 9.336444 & 2.455299 & 4.024227 \\ 1 & 9.701453 & 2.061191 & 4.977106 \\ 1 & 10.180015 & 2.818731 & 3.418530 \\ 1 & 8.815554 & 1.420361 & 3.479850 \\ 6 & 9.136179 & 4.911846 & 6.919390 \\ 6 & 7.987933 & 5.303245 & 7.874361 \\ 1 & 7.187700 & 4.551387 & 7.842942 \\ 1 & 7.542151 & 6.274537 & 7.634937 \\ 6 & 9.400333 & 3.415710 & 7.161037 \\ 1 & 10.320542 & 3.081904 & 6.663229 \\ 1 & 8.571091 & 2.776164 & 6.822151 \\ 6 & 10.443559 & 5.619134 & 7.317385 \\ 1 & 10.343195 & 6.704366 & 7.411203 \\ 1 & 11.234459 & 5.408185 & 6.582068 \\ 1 & 10.777374 & 5.238884 & 8.294713 \\ 1 & 8.362237 & 5.336203 & 8.909392 \\ 1 & 9.520025 & 3.245334 & 8.240885 \\ 7 & 8.426724 & 0.080997 & 3.009498 \\ 14 & 7.549938 & -0.804148 & 4.159622 \\ 14 & 9.154455 & -0.355622 & 1.539180 \\ 6 & 10.521780 & 0.892522 & 1.120458 \\ 1 & 11.342317 & 0.810553 & 1.851355 \\ 1 & 10.940909 & 0.725168 & 0.115520 \\ 1 & 10.140274 & 1.923185 & 1.167778 \\ 6 & 7.956334 & -0.389665 & 0.053315 \\ 1 & 7.138841 & -1.102863 & 0.248035 \\ 1 & 7.493709 & 0.589758 & -0.149134 \\ 1 & 8.468631 & -0.711456 & -0.867756 \\ 6 & 9.987124 & -2.060486 & 1.520707\end{array}$




$\begin{array}{lccc}1 & 10.539143 & -2.210740 & 0.578752 \\ 1 & 10.700164 & -2.155259 & 2.354304 \\ 1 & 9.254072 & -2.876018 & 1.613465 \\ 6 & 8.073270 & -2.613129 & 4.383310 \\ 1 & 7.821279 & -3.216520 & 3.497454 \\ 1 & 9.158702 & -2.694715 & 4.546515 \\ 1 & 7.559412 & -3.060614 & 5.249344 \\ 6 & 5.674586 & -0.867492 & 3.827821 \\ 1 & 5.145718 & -1.468563 & 4.585023 \\ 1 & 5.233293 & 0.141587 & 3.831426 \\ 1 & 5.475459 & -1.314967 & 2.840536 \\ 6 & 7.748304 & 0.035031 & 5.855013 \\ 1 & 7.103807 & -0.415310 & 6.626304 \\ 1 & 8.793543 & -0.017668 & 6.200120 \\ 1 & 7.473764 & 1.096667 & 5.759740\end{array}$

HMDS:

$\begin{array}{rrrc}7 & -0.688365 & -0.061491 & 0.026697 \\ 14 & -1.688732 & 1.368215 & 0.127669 \\ 14 & 1.048108 & -0.201427 & -0.122556 \\ 6 & 1.518032 & -1.962371 & 0.336737 \\ 1 & 1.044021 & -2.689371 & -0.340623 \\ 1 & 2.606808 & -2.105583 & 0.267948 \\ 1 & 1.208205 & -2.199279 & 1.365597 \\ 6 & 1.882778 & 1.015945 & 1.045318 \\ 1 & 1.596529 & 2.055007 & 0.819064 \\ 1 & 1.603557 & 0.808116 & 2.089276 \\ 1 & 2.978479 & 0.950459 & 0.962214 \\ 6 & 1.624906 & 0.158431 & -1.877675 \\ 1 & 2.716978 & 0.050689 & -1.967849 \\ 1 & 1.152128 & -0.531760 & -2.592588 \\ 1 & 1.360501 & 1.184057 & -2.175819 \\ 6 & -1.018523 & 2.673155 & -1.051359 \\ 1 & 0.012926 & 2.961010 & -0.794323 \\ 1 & -1.018772 & 2.301384 & -2.087015 \\ 1 & -1.633917 & 3.584876 & -1.012698 \\ 6 & -1.712420 & 2.084616 & 1.868886 \\ 1 & -2.359207 & 2.973836 & 1.926175 \\ 1 & -2.087211 & 1.342793 & 2.590338 \\ 1 & -0.700948 & 2.379931 & 2.186543 \\ 6 & -3.438209 & 0.883701 & -0.360832 \\ 1 & -4.113113 & 1.751523 & -0.312274 \\ 1 & -3.467000 & 0.485179 & -1.385717 \\ 1 & -3.838904 & 0.114238 & 0.317195 \\ 1 & -1.186402 & -0.944823 & 0.101373\end{array}$




\section{IN1:}

$\begin{array}{cccc}15 & 2.413583 & 1.214670 & 10.999039 \\ 7 & 2.108003 & 3.805072 & 10.491908 \\ 6 & 0.205512 & 0.552372 & 12.766590 \\ 1 & 0.673646 & 1.384560 & 13.319650 \\ 6 & 3.129483 & 2.454564 & 12.177223 \\ 6 & 3.975142 & 2.049758 & 13.407018 \\ 6 & 0.031286 & 0.144954 & 10.277637 \\ 1 & -0.917926 & -0.359544 & 10.496304 \\ 6 & 4.840456 & 0.807349 & 13.119562 \\ 1 & 4.243566 & -0.076460 & 12.874825 \\ 1 & 5.525474 & 0.990870 & 12.278045 \\ 6 & 0.726385 & 0.618956 & 11.345094 \\ 6 & 3.355179 & -0.094869 & 10.569555 \\ 1 & 4.423587 & 0.041079 & 10.407304 \\ 1 & 2.846171 & -0.914587 & 10.063105 \\ 6 & 3.085796 & 1.700021 & 14.614688 \\ 1 & 2.375228 & 2.500029 & 14.862518 \\ 1 & 2.509984 & 0.785679 & 14.417495 \\ 6 & 2.736599 & 3.695398 & 11.758042 \\ 6 & 1.619023 & 2.337348 & 8.546092 \\ 6 & 4.989143 & 3.135866 & 13.803701 \\ 1 & 5.579125 & 3.452353 & 12.930839 \\ 1 & 4.540174 & 4.021175 & 14.261523 \\ 6 & 1.081506 & 1.065729 & 8.138618 \\ 1 & 1.123333 & 0.857280 & 7.065588 \\ 6 & 1.912851 & 2.573306 & 9.863895 \\ 6 & 2.734302 & 5.111893 & 12.370105 \\ 6 & 2.001305 & 3.312235 & 7.436638 \\ 1 & 2.243240 & 4.269170 & 7.913498 \\ 6 & 2.229556 & 5.138455 & 13.829654 \\ 1 & 1.175783 & 4.815268 & 13.826694 \\ 1 & 2.760674 & 4.399983 & 14.436503 \\ 6 & 0.673360 & -0.763068 & 13.407361 \\ 1 & 0.152530 & -1.609715 & 12.934078 \\ 1 & 0.455451 & -0.777728 & 14.486178 \\ 1 & 1.751444 & -0.917133 & 13.255215 \\ 6 & 0.374780 & 0.153294 & 8.873993 \\ 1 & -0.085144 & -0.656140 & 8.299130 \\ 6 & 1.001828 & 4.796888 & 10.619641 \\ 6 & 4.100498 & 5.827045 & 12.200165 \\ 1 & 3.908127 & 6.907800 & 12.298988 \\ 1 & 4.780840 & 5.568835 & 13.020588 \\ 6 & -1.309046 & 0.713217 & 12.884882 \\ 1 & -1.654121 & 1.632628 & 12.390272 \\ 1 & -1.603411 & 0.756069 & 13.943786\end{array}$




$\begin{array}{rrrc}1 & -1.840858 & -0.137704 & 12.433717 \\ 6 & 1.696557 & 5.850127 & 11.481475 \\ 1 & 0.969430 & 6.412700 & 12.084082 \\ 1 & 2.199467 & 6.570471 & 10.820490 \\ 6 & 0.512644 & 5.404008 & 9.315361 \\ 1 & 1.323869 & 5.897749 & 8.763476 \\ 1 & -0.245634 & 6.164060 & 9.555217 \\ 1 & 0.040935 & 4.648750 & 8.675142 \\ 6 & 2.341853 & 6.501172 & 14.507375 \\ 1 & 1.863256 & 7.297820 & 13.917897 \\ 1 & 3.391188 & 6.788207 & 14.664538 \\ 1 & 1.855107 & 6.479885 & 15.492187 \\ 6 & -0.184782 & 4.135531 & 11.335380 \\ 1 & -0.636596 & 3.367998 & 10.688571 \\ 1 & -0.951161 & 4.884932 & 11.581535 \\ 1 & 0.135728 & 3.641524 & 12.266003 \\ 6 & 4.789130 & 5.555686 & 10.867048 \\ 1 & 4.128429 & 5.776861 & 10.015303 \\ 1 & 5.080716 & 4.498336 & 10.780057 \\ 1 & 5.694638 & 6.170011 & 10.766820 \\ 6 & 3.296993 & 2.802121 & 6.788639 \\ 1 & 4.090248 & 2.688924 & 7.540714 \\ 1 & 3.642163 & 3.501153 & 6.011617 \\ 1 & 3.142531 & 1.820448 & 6.314916 \\ 6 & 0.927728 & 3.536073 & 6.369632 \\ 1 & 0.771279 & 2.626721 & 5.770437 \\ 1 & 1.236045 & 4.333922 & 5.677457 \\ 1 & -0.043257 & 3.814315 & 6.802190 \\ 1 & 5.685457 & 2.713956 & 14.543455 \\ 1 & 3.714937 & 1.507268 & 15.498055 \\ 1 & 5.447960 & 0.593499 & 14.011869\end{array}$

TS2:

$\begin{array}{rrrr}15 & 2.486164 & 1.107702 & 11.021089 \\ 7 & 1.817345 & 3.626019 & 10.633453 \\ 6 & 0.135159 & 1.235394 & 12.673975 \\ 1 & 0.624264 & 2.146937 & 13.056399 \\ 6 & 3.240098 & 2.403710 & 12.088789 \\ 6 & 4.378760 & 2.008185 & 13.046830 \\ 6 & -0.065516 & 0.143008 & 10.407637 \\ 1 & -0.897690 & -0.393122 & 10.875691 \\ 6 & 5.546854 & 1.441599 & 12.211579 \\ 1 & 5.267522 & 0.503607 & 11.720337 \\ 1 & 5.868452 & 2.167641 & 11.449016 \\ 6 & 0.721750 & 0.937060 & 11.296881 \\ 6 & 3.275174 & -0.267290 & 10.519425\end{array}$




$\begin{array}{rrrc}1 & 4.276384 & -0.218523 & 10.095438 \\ 1 & 2.612349 & -1.073337 & 10.203386 \\ 6 & 3.906702 & 0.915371 & 14.017070 \\ 1 & 3.078859 & 1.273954 & 14.648645 \\ 1 & 3.581440 & 0.023220 & 13.463461 \\ 6 & 2.648790 & 3.598011 & 11.769910 \\ 6 & 1.367780 & 2.122141 & 8.659612 \\ 6 & 4.987949 & 3.145432 & 13.873076 \\ 1 & 5.506937 & 3.867197 & 13.231055 \\ 1 & 4.265050 & 3.676173 & 14.502833 \\ 6 & 0.791878 & 0.946533 & 8.236550 \\ 1 & 0.781013 & 0.758097 & 7.160304 \\ 6 & 1.608589 & 2.383369 & 10.050221 \\ 6 & 2.558538 & 4.973303 & 12.454862 \\ 6 & 2.076322 & 3.014027 & 7.639597 \\ 1 & 2.324459 & 3.958206 & 8.136213 \\ 6 & 1.911672 & 4.798919 & 13.853972 \\ 1 & 0.867720 & 4.481324 & 13.706224 \\ 1 & 2.393630 & 3.966215 & 14.383523 \\ 6 & 0.475128 & 0.079818 & 13.625768 \\ 1 & -0.023687 & -0.840103 & 13.284336 \\ 1 & 0.126290 & 0.299278 & 14.646583 \\ 1 & 1.551378 & -0.118429 & 13.659493 \\ 6 & 0.056654 & 0.040335 & 9.046527 \\ 1 & -0.562003 & -0.698321 & 8.532550 \\ 6 & 0.862766 & 4.759943 & 10.624753 \\ 6 & 3.877887 & 5.779422 & 12.512749 \\ 1 & 3.593626 & 6.837768 & 12.631893 \\ 1 & 4.453792 & 5.532567 & 13.410775 \\ 6 & -1.383113 & 1.449092 & 12.680528 \\ 1 & -1.725141 & 2.081974 & 11.853771 \\ 1 & -1.695754 & 1.913030 & 13.628111 \\ 1 & -1.912811 & 0.488320 & 12.602880 \\ 6 & 1.589597 & 5.756199 & 11.533132 \\ 1 & 0.878427 & 6.356455 & 12.119285 \\ 1 & 2.157687 & 6.456654 & 10.904547 \\ 6 & 0.632421 & 5.371379 & 9.245347 \\ 1 & 1.568622 & 5.728671 & 8.795791 \\ 1 & -0.040613 & 6.233975 & 9.358666 \\ 1 & 0.153094 & 4.655560 & 8.564558 \\ 6 & 1.935257 & 6.049827 & 14.726537 \\ 1 & 1.488782 & 6.915199 & 14.213439 \\ 1 & 2.959241 & 6.325518 & 15.015176 \\ 1 & 1.364998 & 5.882843 & 15.650631 \\ 6 & -0.492382 & 4.317253 & 11.176743 \\ 1 & -0.914721 & 3.532616 & 10.531558\end{array}$




$\begin{array}{lrrc}1 & -1.188224 & 5.168536 & 11.196717 \\ 1 & -0.406510 & 3.914442 & 12.193397 \\ 6 & 4.748884 & 5.624102 & 11.271884 \\ 1 & 4.185871 & 5.846190 & 10.352051 \\ 1 & 5.123147 & 4.594156 & 11.176710 \\ 1 & 5.613378 & 6.301445 & 11.307491 \\ 6 & 3.412403 & 2.351021 & 7.274268 \\ 1 & 4.007197 & 2.145713 & 8.176690 \\ 1 & 3.999903 & 2.997921 & 6.604679 \\ 1 & 3.240051 & 1.389722 & 6.767558 \\ 6 & 1.262718 & 3.318057 & 6.382471 \\ 1 & 1.083719 & 2.405122 & 5.795556 \\ 1 & 1.807936 & 4.020440 & 5.734251 \\ 1 & 0.282882 & 3.756425 & 6.619491 \\ 1 & 5.743509 & 2.708856 & 14.542757 \\ 1 & 4.737620 & 0.618229 & 14.675159 \\ 1 & 6.404003 & 1.235545 & 12.871371\end{array}$

IN2:

$\begin{array}{cccc}15 & 2.523649 & 1.032898 & 11.041725 \\ 7 & 1.571312 & 3.517091 & 10.800871 \\ 6 & 0.068257 & 1.423058 & 12.504079 \\ 1 & 0.460471 & 2.391883 & 12.845119 \\ 6 & 3.233698 & 2.370158 & 12.047138 \\ 6 & 4.471444 & 2.000854 & 12.893520 \\ 6 & -0.057022 & 0.089753 & 10.371675 \\ 1 & -0.618629 & -0.638431 & 10.960525 \\ 6 & 5.607021 & 1.542992 & 11.953743 \\ 1 & 5.338465 & 0.626689 & 11.415936 \\ 1 & 5.844186 & 2.330288 & 11.221588 \\ 6 & 0.704843 & 1.118316 & 11.136869 \\ 6 & 3.256072 & -0.319339 & 10.417420 \\ 1 & 4.172972 & -0.264342 & 9.833900 \\ 1 & 2.618670 & -1.199462 & 10.335972 \\ 6 & 4.127451 & 0.843744 & 13.848326 \\ 1 & 3.385902 & 1.166460 & 14.595269 \\ 1 & 3.735024 & -0.026422 & 13.302699 \\ 6 & 2.547947 & 3.538226 & 11.790806 \\ 6 & 1.229035 & 2.094471 & 8.746686 \\ 6 & 5.070244 & 3.122152 & 13.749162 \\ 1 & 5.549138 & 3.890187 & 13.131257 \\ 1 & 4.343894 & 3.594475 & 14.421747 \\ 6 & 0.597100 & 1.024898 & 8.209168 \\ 1 & 0.646581 & 0.850711 & 7.133323 \\ 6 & 1.286358 & 2.256554 & 10.211073 \\ 6 & 2.510991 & 4.925241 & 12.459931\end{array}$




$\begin{array}{rrrc}6 & 2.165616 & 2.952316 & 7.907544 \\ 1 & 2.339731 & 3.888751 & 8.447646 \\ 6 & 1.915390 & 4.770887 & 13.882921 \\ 1 & 0.872272 & 4.433026 & 13.779132 \\ 1 & 2.433152 & 3.955676 & 14.407521 \\ 6 & 0.473426 & 0.371674 & 13.536537 \\ 1 & 0.177905 & -0.635738 & 13.202574 \\ 1 & -0.023701 & 0.562469 & 14.499463 \\ 1 & 1.557207 & 0.368966 & 13.701756 \\ 6 & -0.116525 & 0.059615 & 9.031195 \\ 1 & -0.705590 & -0.715035 & 8.536563 \\ 6 & 0.707660 & 4.719018 & 10.735177 \\ 6 & 3.837848 & 5.718159 & 12.471564 \\ 1 & 3.565571 & 6.780201 & 12.588008 \\ 1 & 4.433361 & 5.477682 & 13.358022 \\ 6 & -1.464329 & 1.496996 & 12.436495 \\ 1 & -1.826493 & 1.977408 & 11.520364 \\ 1 & -1.860268 & 2.050502 & 13.301182 \\ 1 & -1.901750 & 0.488275 & 12.467826 \\ 6 & 1.527251 & 5.714320 & 11.561593 \\ 1 & 0.879233 & 6.369386 & 12.161811 \\ 1 & 2.092326 & 6.363789 & 10.878540 \\ 6 & 0.490314 & 5.246631 & 9.314553 \\ 1 & 1.426661 & 5.575925 & 8.846437 \\ 1 & -0.182131 & 6.115428 & 9.364067 \\ 1 & 0.015745 & 4.487173 & 8.676480 \\ 6 & 1.950387 & 6.035716 & 14.735580 \\ 1 & 1.471635 & 6.886987 & 14.228314 \\ 1 & 2.979818 & 6.330731 & 14.982785 \\ 1 & 1.417137 & 5.873640 & 15.682418 \\ 6 & -0.681993 & 4.461296 & 11.325092 \\ 1 & -1.212837 & 3.719369 & 10.713486 \\ 1 & -1.265726 & 5.392940 & 11.309991 \\ 1 & -0.643341 & 4.098240 & 12.359056 \\ 6 & 4.675005 & 5.546250 & 11.209883 \\ 1 & 4.104267 & 5.808958 & 10.305608 \\ 1 & 5.001418 & 4.503065 & 11.090401 \\ 1 & 5.567653 & 6.186880 & 11.237022 \\ 6 & 3.536580 & 2.268341 & 7.796985 \\ 1 & 3.985864 & 2.112480 & 8.788807 \\ 1 & 4.224136 & 2.893025 & 7.206192 \\ 1 & 3.444028 & 1.286255 & 7.310509 \\ 6 & 1.615644 & 3.284658 & 6.522576 \\ 1 & 1.543240 & 2.383439 & 5.895343 \\ 1 & 2.285453 & 3.987168 & 6.005124 \\ 1 & 0.614850 & 3.737046 & 6.578397\end{array}$




$\begin{array}{llll}1 & 5.857655 & 2.681003 & 14.378255 \\ 1 & 5.033298 & 0.522233 & 14.384917 \\ 1 & 6.515179 & 1.337324 & 12.542044\end{array}$

TS3:

$\begin{array}{cccc}15 & 2.446928 & 0.949934 & 11.132810 \\ 7 & 1.440417 & 3.542350 & 10.832844 \\ 6 & -0.073769 & 1.421096 & 12.420514 \\ 1 & 0.324862 & 2.371302 & 12.805293 \\ 6 & 3.181319 & 2.374894 & 11.968770 \\ 6 & 4.415887 & 2.004498 & 12.834473 \\ 6 & -0.103470 & 0.119085 & 10.256367 \\ 1 & -0.699149 & -0.613175 & 10.803808 \\ 6 & 5.535171 & 1.429430 & 11.940454 \\ 1 & 5.249884 & 0.470365 & 11.494892 \\ 1 & 5.786339 & 2.133642 & 11.131899 \\ 6 & 0.591330 & 1.163911 & 11.055192 \\ 6 & 3.194797 & -0.221119 & 10.234540 \\ 1 & 4.119705 & -0.052608 & 9.687015 \\ 1 & 2.657448 & -1.161558 & 10.115016 \\ 6 & 4.015388 & 0.936244 & 13.869569 \\ 1 & 3.258249 & 1.333354 & 14.563893 \\ 1 & 3.612259 & 0.033438 & 13.387710 \\ 6 & 2.475835 & 3.544273 & 11.755625 \\ 6 & 1.235085 & 2.156978 & 8.774529 \\ 6 & 5.076016 & 3.146930 & 13.613916 \\ 1 & 5.581463 & 3.851227 & 12.943811 \\ 1 & 4.385512 & 3.692514 & 14.265913 \\ 6 & 0.769467 & 1.021406 & 8.170808 \\ 1 & 0.957254 & 0.848694 & 7.110618 \\ 6 & 1.083311 & 2.324302 & 10.202368 \\ 6 & 2.526163 & 4.945777 & 12.401503 \\ 6 & 2.153038 & 3.099949 & 8.008666 \\ 1 & 2.270815 & 4.010908 & 8.604131 \\ 6 & 2.000598 & 4.853908 & 13.856187 \\ 1 & 0.940944 & 4.558509 & 13.813811 \\ 1 & 2.506828 & 4.029937 & 14.377822 \\ 6 & 0.266677 & 0.332343 & 13.436924 \\ 1 & -0.003496 & -0.662819 & 13.049177 \\ 1 & -0.295257 & 0.491483 & 14.369242 \\ 1 & 1.336966 & 0.324566 & 13.678394 \\ 6 & 0.003298 & 0.053442 & 8.914632 \\ 1 & -0.504265 & -0.749651 & 8.375481 \\ 6 & 0.613333 & 4.774624 & 10.803994 \\ 6 & 3.877727 & 5.695640 & 12.310000 \\ 1 & 3.638956 & 6.768569 & 12.395328\end{array}$




$\begin{array}{rrrc}1 & 4.516182 & 5.478902 & 13.171013 \\ 6 & -1.599065 & 1.528894 & 12.281112 \\ 1 & -1.900073 & 2.014209 & 11.344934 \\ 1 & -2.027803 & 2.091612 & 13.123554 \\ 1 & -2.056692 & 0.528551 & 12.286240 \\ 6 & 1.532174 & 5.754649 & 11.531407 \\ 1 & 0.964319 & 6.472892 & 12.140284 \\ 1 & 2.089222 & 6.337841 & 10.784726 \\ 6 & 0.283554 & 5.257199 & 9.389199 \\ 1 & 1.170448 & 5.601344 & 8.843202 \\ 1 & -0.415542 & 6.102969 & 9.459419 \\ 1 & -0.205529 & 4.460340 & 8.809532 \\ 6 & 2.135164 & 6.136159 & 14.672035 \\ 1 & 1.665712 & 6.994804 & 14.168651 \\ 1 & 3.188427 & 6.391828 & 14.854067 \\ 1 & 1.649011 & 6.020968 & 15.650668 \\ 6 & -0.729357 & 4.590368 & 11.522517 \\ 1 & -1.369942 & 3.906542 & 10.951795 \\ 1 & -1.243531 & 5.560466 & 11.584478 \\ 1 & -0.620218 & 4.196092 & 12.539894 \\ 6 & 4.640151 & 5.450808 & 11.013128 \\ 1 & 4.023594 & 5.681349 & 10.130368 \\ 1 & 4.945199 & 4.398456 & 10.922806 \\ 1 & 5.541105 & 6.078405 & 10.962817 \\ 6 & 3.556419 & 2.488645 & 7.897235 \\ 1 & 3.977564 & 2.294469 & 8.894656 \\ 1 & 4.230675 & 3.180835 & 7.369075 \\ 1 & 3.528844 & 1.536001 & 7.348126 \\ 6 & 1.616374 & 3.485202 & 6.631232 \\ 1 & 1.577111 & 2.613510 & 5.961038 \\ 1 & 2.273669 & 4.231210 & 6.159892 \\ 1 & 0.602611 & 3.906535 & 6.692546 \\ 1 & 5.851342 & 2.706542 & 14.257886 \\ 1 & 4.895470 & 0.633559 & 14.457622 \\ 1 & 6.440983 & 1.266614 & 12.545002 \\ & & & \\ 4: & & & \\ 15 & 2.132081 & 0.624912 & 12.019375 \\ 7 & 0.826019 & 3.162355 & 11.150067 \\ 6 & -1.336925 & 1.151419 & 11.748346 \\ 1 & -0.891660 & 1.880163 & 12.437565 \\ 6 & 2.954151 & 2.300063 & 12.009233 \\ 6 & 4.365146 & 2.220557 & 12.676900 \\ 6 & -0.777143 & 0.181608 & 9.522808 \\ 1 & -1.549556 & -0.560132 & 9.734978 \\ 6 & 5.322731 & 1.342264 & 11.843512\end{array}$




$\begin{array}{cccc}1 & 4.969071 & 0.311446 & 11.741387 \\ 1 & 5.471665 & 1.766228 & 10.838448 \\ 6 & -0.514563 & 1.176515 & 10.468407 \\ 6 & 2.776041 & -0.321030 & 10.816878 \\ 1 & 3.495310 & -0.001707 & 10.058364 \\ 1 & 2.418838 & -1.352746 & 10.739169 \\ 6 & 4.215551 & 1.569315 & 14.070953 \\ 1 & 3.537368 & 2.156891 & 14.708272 \\ 1 & 3.822967 & 0.543998 & 14.014670 \\ 6 & 2.121071 & 3.351998 & 11.725973 \\ 6 & 1.168920 & 2.098702 & 8.953475 \\ 6 & 5.143456 & 3.522716 & 12.897847 \\ 1 & 5.358289 & 4.039177 & 11.955957 \\ 1 & 4.651371 & 4.209923 & 13.592024 \\ 6 & 0.902880 & 1.057877 & 8.061639 \\ 1 & 1.454477 & 1.013690 & 7.119870 \\ 6 & 0.494566 & 2.137396 & 10.196103 \\ 6 & 2.223585 & 4.866771 & 12.112848 \\ 6 & 2.196569 & 3.131488 & 8.532122 \\ 1 & 2.217879 & 3.907214 & 9.304829 \\ 6 & 2.067531 & 4.960635 & 13.654896 \\ 1 & 1.111176 & 4.505294 & 13.940066 \\ 1 & 2.830315 & 4.322708 & 14.120588 \\ 6 & -1.326795 & -0.210765 & 12.452266 \\ 1 & -1.813968 & -0.984761 & 11.840730 \\ 1 & -1.886998 & -0.142410 & 13.397107 \\ 1 & -0.305438 & -0.537805 & 12.682425 \\ 6 & -0.059429 & 0.095635 & 8.337895 \\ 1 & -0.259923 & -0.706510 & 7.625886 \\ 6 & -0.038851 & 4.370400 & 11.230517 \\ 6 & 3.432399 & 5.731256 & 11.663959 \\ 1 & 3.070786 & 6.773144 & 11.686832 \\ 1 & 4.231288 & 5.703979 & 12.408586 \\ 6 & -2.793711 & 1.542764 & 11.457661 \\ 1 & -2.874599 & 2.469839 & 10.874089 \\ 1 & -3.356778 & 1.673132 & 12.394714 \\ 1 & -3.289190 & 0.749393 & 10.876789 \\ 6 & 1.004458 & 5.468789 & 11.397850 \\ 1 & 0.610062 & 6.346719 & 11.928728 \\ 1 & 1.312894 & 5.807662 & 10.396039 \\ 6 & -0.832755 & 4.600128 & 9.945038 \\ 1 & -0.165994 & 4.658149 & 9.074525 \\ 1 & -1.377742 & 5.551948 & 10.030987 \\ 1 & -1.561924 & 3.799276 & 9.763377 \\ 6 & 2.152067 & 6.364067 & 14.249256 \\ 1 & 1.416518 & 7.049758 & 13.802311\end{array}$




$\begin{array}{cccc}1 & 3.147823 & 6.810012 & 14.115124 \\ 1 & 1.950971 & 6.329322 & 15.329066 \\ 6 & -1.029569 & 4.351045 & 12.412694 \\ 1 & -1.934319 & 3.783676 & 12.168754 \\ 1 & -1.340656 & 5.379659 & 12.649308 \\ 1 & -0.591021 & 3.905703 & 13.313290 \\ 6 & 4.019162 & 5.456778 & 10.285665 \\ 1 & 3.314525 & 5.699083 & 9.476107 \\ 1 & 4.303815 & 4.401294 & 10.174879 \\ 1 & 4.916652 & 6.071981 & 10.126739 \\ 6 & 3.596392 & 2.518776 & 8.439289 \\ 1 & 3.905612 & 2.111208 & 9.411241 \\ 1 & 4.332661 & 3.275155 & 8.126623 \\ 1 & 3.617635 & 1.703580 & 7.699057 \\ 6 & 1.826670 & 3.801141 & 7.203706 \\ 1 & 1.892428 & 3.089410 & 6.366946 \\ 1 & 2.522348 & 4.626695 & 6.988539 \\ 1 & 0.803958 & 4.204085 & 7.215668 \\ 1 & 6.111024 & 3.258272 & 13.349473 \\ 1 & 5.198782 & 1.519194 & 14.563851 \\ 1 & 6.304340 & 1.310826 & 12.340207\end{array}$

TS4:

$\begin{array}{cccc}15 & 2.123026 & 0.534587 & 11.407873 \\ 7 & 0.856460 & 3.400044 & 10.909406 \\ 6 & -0.849898 & 1.238905 & 12.035285 \\ 1 & -0.500720 & 2.146423 & 12.543940 \\ 6 & 2.768637 & 2.248803 & 11.889979 \\ 6 & 4.026723 & 2.011116 & 12.790928 \\ 6 & -0.401459 & 0.006223 & 9.890766 \\ 1 & -1.098118 & -0.749705 & 10.257387 \\ 6 & 5.029914 & 1.066287 & 12.090043 \\ 1 & 4.641084 & 0.050421 & 11.954707 \\ 1 & 5.305790 & 1.458796 & 11.098249 \\ 6 & -0.039218 & 1.102047 & 10.747008 \\ 6 & 2.758027 & 0.132279 & 9.857627 \\ 1 & 3.573971 & 0.699033 & 9.408672 \\ 1 & 2.730791 & -0.928056 & 9.593280 \\ 6 & 3.572572 & 1.343822 & 14.107798 \\ 1 & 2.902066 & 2.009218 & 14.672070 \\ 1 & 3.038401 & 0.401228 & 13.924728 \\ 6 & 2.036059 & 3.394186 & 11.678569 \\ 6 & 1.142274 & 2.135579 & 8.867270 \\ 6 & 4.876310 & 3.225353 & 13.186407 \\ 1 & 5.316308 & 3.721041 & 12.311842 \\ 1 & 4.331173 & 3.958278 & 13.785394\end{array}$




$\begin{array}{cccc}6 & 1.213800 & 0.820350 & 8.332360 \\ 1 & 1.752631 & 0.673287 & 7.393049 \\ 6 & 0.545922 & 2.267255 & 10.110650 \\ 6 & 2.204077 & 4.859332 & 12.198824 \\ 6 & 1.909525 & 3.247483 & 8.179879 \\ 1 & 1.870406 & 4.121208 & 8.843452 \\ 6 & 1.929439 & 4.903134 & 13.724299 \\ 1 & 0.878686 & 4.630341 & 13.892173 \\ 1 & 2.506412 & 4.115225 & 14.224645 \\ 6 & -0.702445 & 0.070114 & 13.012432 \\ 1 & -0.882723 & -0.894218 & 12.512178 \\ 1 & -1.442997 & 0.169989 & 13.819870 \\ 1 & 0.297948 & 0.031474 & 13.459689 \\ 6 & 0.227104 & -0.141953 & 8.695113 \\ 1 & 0.060389 & -1.019518 & 8.067802 \\ 6 & 0.030727 & 4.616673 & 11.005851 \\ 6 & 3.516235 & 5.609276 & 11.838114 \\ 1 & 3.265092 & 6.682937 & 11.865919 \\ 1 & 4.282751 & 5.484343 & 12.606041 \\ 6 & -2.335016 & 1.401883 & 11.671911 \\ 1 & -2.481158 & 2.092555 & 10.829513 \\ 1 & -2.909558 & 1.770471 & 12.535109 \\ 1 & -2.763948 & 0.432248 & 11.375981 \\ 6 & 1.092188 & 5.623152 & 11.438467 \\ 1 & 0.677413 & 6.438779 & 12.048723 \\ 1 & 1.521140 & 6.080125 & 10.534100 \\ 6 & -0.598987 & 4.987292 & 9.662555 \\ 1 & 0.158455 & 5.203644 & 8.898850 \\ 1 & -1.222783 & 5.884056 & 9.787618 \\ 1 & -1.240751 & 4.172288 & 9.294240 \\ 6 & 2.211261 & 6.243040 & 14.398986 \\ 1 & 1.681559 & 7.071517 & 13.904684 \\ 1 & 3.283367 & 6.485730 & 14.394641 \\ 1 & 1.882696 & 6.219857 & 15.447381 \\ 6 & -1.114158 & 4.532594 & 12.032247 \\ 1 & -1.910584 & 3.865530 & 11.683319 \\ 1 & -1.551951 & 5.533324 & 12.164098 \\ 1 & -0.777340 & 4.177588 & 13.013295 \\ 6 & 4.106515 & 5.274683 & 10.475759 \\ 1 & 3.395464 & 5.468510 & 9.657947 \\ 1 & 4.385488 & 4.213550 & 10.418708 \\ 1 & 5.003815 & 5.879533 & 10.281193 \\ 6 & 3.389871 & 2.918770 & 7.966330 \\ 1 & 3.890420 & 2.711436 & 8.921795 \\ 1 & 3.898900 & 3.773682 & 7.495435 \\ 1 & 3.524236 & 2.048322 & 7.305200\end{array}$




$\begin{array}{cccc}6 & 1.244791 & 3.612643 & 6.847950 \\ 1 & 1.298001 & 2.767554 & 6.143834 \\ 1 & 1.749922 & 4.471789 & 6.380627 \\ 1 & 0.183471 & 3.863861 & 6.985275 \\ 1 & 5.708528 & 2.863371 & 13.807913 \\ 1 & 4.447096 & 1.118604 & 14.738928 \\ 1 & 5.945275 & 0.995370 & 12.696684\end{array}$

5:

$\begin{array}{llll}15 & 2.003479 & 0.569789 & 11.398835\end{array}$

$\begin{array}{lllll}7 & 0.874533 & 3.463777 & 10.869326\end{array}$

$\begin{array}{llll}6 & -0.713067 & 1.256290 & 12.059727\end{array}$

$\begin{array}{llll}1 & -0.393831 & 2.180378 & 12.560020\end{array}$

$\begin{array}{llll}6 & 2.683712 & 2.248309 & 11.937341\end{array}$

$\begin{array}{llll}6 & 3.939090 & 1.966350 & 12.818551\end{array}$

$\begin{array}{llll}6 & -0.256818 & -0.029833 & 9.940482\end{array}$

$\begin{array}{llll}1 & -1.091173 & -0.662982 & 10.245750\end{array}$

$\begin{array}{llll}6 & 4.882481 & 0.992003 & 12.077218\end{array}$

$14.411081 \quad 0.024846 \quad 11.852860$

$\begin{array}{lllll}1 & 5.235489 & 1.436360 & 11.132742\end{array}$

$\begin{array}{llll}6 & 0.231758 & 1.049873 & 10.861005\end{array}$

$\begin{array}{llll}6 & 2.627309 & 0.292180 & 9.640787\end{array}$

$\begin{array}{lllll}1 & 3.553276 & 0.864402 & 9.504387\end{array}$

$\begin{array}{llll}1 & 2.865827 & -0.776203 & 9.529929\end{array}$

$\begin{array}{llll}6 & 3.490890 & 1.302099 & 14.137355\end{array}$

$\begin{array}{llll}1 & 2.837101 & 1.977388 & 14.709653\end{array}$

$\begin{array}{llll}1 & 2.939255 & 0.370848 & 13.950481\end{array}$

$\begin{array}{lllll}6 & 1.999921 & 3.422461 & 11.704860\end{array}$

$\begin{array}{llll}6 & 1.238619 & 2.142724 & 8.896811\end{array}$

$\begin{array}{llll}6 & 4.843759 & 3.144252 & 13.199770\end{array}$

$\begin{array}{llll}1 & 5.256079 & 3.648738 & 12.317141\end{array}$

$\begin{array}{lllll}1 & 4.350850 & 3.879618 & 13.842225\end{array}$

$\begin{array}{llll}6 & 1.554799 & 0.705133 & 8.564133\end{array}$

$\begin{array}{lllll}1 & 1.977057 & 0.596546 & 7.556235\end{array}$

$\begin{array}{llll}6 & 0.627571 & 2.304857 & 10.092118\end{array}$

$\begin{array}{llll}6 & 2.180597 & 4.876003 & 12.242438\end{array}$

$\begin{array}{llll}6 & 1.879004 & 3.244288 & 8.089269\end{array}$

$\begin{array}{lllll}1 & 1.814595 & 4.159364 & 8.694867\end{array}$

$\begin{array}{lllll}6 & 1.919629 & 4.908500 & 13.768287\end{array}$

$\begin{array}{lllll}1 & 0.869159 & 4.636099 & 13.943700\end{array}$

$\begin{array}{lllll}1 & 2.502246 & 4.115026 & 14.254297\end{array}$

$\begin{array}{llll}6 & -0.636809 & 0.127536 & 13.089844\end{array}$

$\begin{array}{lllll}1 & -0.798040 & -0.854280 & 12.616337\end{array}$

$\begin{array}{llll}1 & -1.416296 & 0.262900 & 13.854621\end{array}$

$\begin{array}{lllll}1 & 0.337694 & 0.095741 & 13.594855\end{array}$

$\begin{array}{llll}6 & 0.358296 & -0.178562 & 8.767501\end{array}$ 


\begin{tabular}{|c|c|c|c|}
\hline & 08 & 27 & \\
\hline & 0.028122 & 4.661147 & 11.001302 \\
\hline & 3.508830 & 5.592923 & \\
\hline & 3.292715 & 6.673695 & 11.91860 \\
\hline & 4.278362 & 2222 & 61 \\
\hline & -2.162755 & 1.420020 & 11.584264 \\
\hline & -2.227623 & & \\
\hline & -2.783302 & 164 & \\
\hline & -2.609899 & 442278 & 11. \\
\hline 6 & 1.075715 & & \\
\hline & 0.64 & & \\
\hline & 1.51 & & \\
\hline 6 & 082 & & \\
\hline & 0.181463 & & \\
\hline 1 & -1.235891 & & \\
\hline & -1.1 & & \\
\hline 5 & 2.21 & & \\
\hline & 1.68 & & \\
\hline 1 & 3.28 & & \\
\hline t & & & \\
\hline 6 & -1.1 & & \\
\hline 1 & -1.9 & & \\
\hline 1 & -1.58 & & 12. \\
\hline 1 & -0.7 & & \\
\hline 6 & & & \\
\hline 1 & 3.3 & & \\
\hline 1 & 4.3 & & \\
\hline 1 & $4.9^{\prime}$ & & 672 \\
\hline & & & \\
\hline & 3.9 & & \\
\hline 1 & $3.7 \varepsilon$ & & \\
\hline 1 & 3.52 & & 7.1 \\
\hline 6 & & & \\
\hline & & & \\
\hline & & & \\
\hline 1 & & 635 & 134 \\
\hline 1 & & & 13.771562 \\
\hline & & & \\
\hline & 5.762894 & 0.782538 & 3430 \\
\hline \\
\hline 15 & & & \\
\hline 7 & & & \\
\hline 6 & & & \\
\hline 1 & -0.214889 & 2.593895 & 12. \\
\hline & 4.505388 & 0.567182 & 14.80977 \\
\hline
\end{tabular}




$\begin{array}{rrrc}1 & 4.057881 & -0.199137 & 15.462376 \\ 6 & 5.546467 & 2.824439 & 14.713877 \\ 1 & 5.829069 & 3.717298 & 15.294529 \\ 6 & 6.336182 & 1.052799 & 13.173929 \\ 1 & 7.201091 & 0.630303 & 12.638690 \\ 6 & 2.897282 & 2.375842 & 11.721437 \\ 6 & 4.007176 & 2.075967 & 12.797698 \\ 6 & -0.915939 & 0.291694 & 10.187555 \\ 1 & -1.495735 & -0.401026 & 10.801379 \\ 6 & 5.291625 & 1.491053 & 12.135484 \\ 1 & 5.051511 & 0.609805 & 11.528186 \\ 1 & 5.721572 & 2.245292 & 11.453135 \\ 6 & -0.387641 & 1.441826 & 10.776165 \\ 6 & 2.482797 & -0.370142 & 10.748425 \\ 1 & 2.599325 & -1.143050 & 9.981897 \\ 1 & 2.010839 & -0.694156 & 11.677347 \\ 6 & 3.459231 & 1.009708 & 13.778697 \\ 1 & 2.576131 & 1.420485 & 14.295182 \\ 1 & 3.128231 & 0.130010 & 13.209730 \\ 6 & 4.935535 & 1.775526 & 15.646044 \\ 1 & 5.667753 & 1.473554 & 16.412966 \\ 1 & 4.063973 & 2.198507 & 16.173173 \\ 6 & 2.050520 & 3.449380 & 11.547641 \\ 6 & 0.584218 & 2.048357 & 8.601906 \\ 6 & 4.521432 & 3.254842 & 13.650667 \\ 1 & 5.017228 & 3.975742 & 12.990295 \\ 1 & 3.695507 & 3.761464 & 14.160427 \\ 6 & 0.002854 & 0.897600 & 8.059402 \\ 1 & 0.149837 & 0.680728 & 6.999538 \\ 6 & 5.722086 & -0.019468 & 14.083388 \\ 1 & 5.416042 & -0.891612 & 13.482426 \\ 1 & 6.468935 & -0.370074 & 14.814665 \\ 6 & 6.780429 & 2.246479 & 14.020123 \\ 1 & 7.532112 & 1.933651 & 14.763770 \\ 1 & 7.248700 & 3.012524 & 13.380344 \\ 6 & 0.398963 & 2.319809 & 9.986346 \\ 6 & 2.007203 & 4.821451 & 12.285761 \\ 6 & 1.410954 & 2.907514 & 7.647610 \\ 1 & 1.842604 & 3.728091 & 8.234880 \\ 6 & 1.390304 & 4.622485 & 13.699072 \\ 1 & 0.316803 & 4.418497 & 13.570493 \\ 1 & 1.797182 & 3.714691 & 14.165446 \\ 6 & 0.001920 & 0.556045 & 13.110962 \\ 1 & -0.375156 & -0.444870 & 12.848476 \\ 1 & -0.208202 & 0.727423 & 14.178200 \\ 1 & 1.085894 & 0.570619 & 12.971155\end{array}$




$\begin{array}{rrrr}6 & -0.724882 & 0.012017 & 8.840042 \\ 1 & -1.149616 & -0.891396 & 8.399289 \\ 6 & 0.178838 & 4.729788 & 10.599283 \\ 6 & 3.312622 & 5.667049 & 12.299888 \\ 1 & 2.988802 & 6.717067 & 12.387666 \\ 1 & 3.917021 & 5.494390 & 13.190568 \\ 6 & -2.163790 & 1.671334 & 12.569880 \\ 1 & -2.713746 & 2.375410 & 11.932940 \\ 1 & -2.328276 & 1.955026 & 13.620543 \\ 1 & -2.609541 & 0.675086 & 12.427543 \\ 6 & 1.040621 & 5.664590 & 11.431234 \\ 1 & 0.432270 & 6.331399 & 12.061043 \\ 1 & 1.615519 & 6.307793 & 10.751029 \\ 6 & -0.021416 & 5.319339 & 9.203365 \\ 1 & 0.931516 & 5.473764 & 8.681058 \\ 1 & -0.518367 & 6.295789 & 9.302139 \\ 1 & -0.668865 & 4.674324 & 8.597610 \\ 6 & 1.550188 & 5.809100 & 14.644344 \\ 1 & 1.177611 & 6.741301 & 14.192946 \\ 1 & 2.601471 & 5.972438 & 14.920893 \\ 1 & 0.985883 & 5.640994 & 15.572076 \\ 6 & -1.229713 & 4.556341 & 11.188210 \\ 1 & -1.788636 & 3.788865 & 10.635100 \\ 1 & -1.770021 & 5.507833 & 11.081911 \\ 1 & -1.229510 & 4.293239 & 12.251603 \\ 6 & 4.167888 & 5.517949 & 11.046875 \\ 1 & 3.582279 & 5.701875 & 10.132309 \\ 1 & 4.577614 & 4.501919 & 10.959893 \\ 1 & 5.004243 & 6.231308 & 11.056220 \\ 6 & 2.573553 & 2.146895 & 6.991803 \\ 1 & 3.296298 & 1.779922 & 7.727623 \\ 1 & 3.095161 & 2.815574 & 6.290429 \\ 1 & 2.203568 & 1.287960 & 6.411468 \\ 6 & 0.543922 & 3.473009 & 6.509727 \\ 1 & 0.296327 & 2.673669 & 5.794205 \\ 1 & 1.098395 & 4.245771 & 5.956096 \\ 1 & -0.402677 & 3.907034 & 6.850784\end{array}$

\section{TS4':}

$\begin{array}{cccc}15 & 2.710091 & 1.049281 & 10.293057 \\ 7 & 0.731403 & 3.467969 & 10.867590 \\ 6 & -1.031518 & 1.441125 & 12.148755 \\ 1 & -0.835109 & 2.459816 & 12.493833 \\ 6 & 4.389225 & 0.747486 & 14.770478 \\ 1 & 3.893158 & 0.140165 & 15.544280 \\ 6 & 5.731457 & 2.801924 & 14.322644\end{array}$




$\begin{array}{rrrc}1 & 6.182392 & 3.701744 & 14.770906 \\ 6 & 6.139830 & 0.761226 & 12.981798 \\ 1 & 6.891569 & 0.154496 & 12.452826 \\ 6 & 2.795739 & 2.390742 & 11.612676 \\ 6 & 3.958258 & 2.069656 & 12.617717 \\ 6 & -0.318913 & 0.005016 & 10.198708 \\ 1 & -0.825537 & -0.817518 & 10.710308 \\ 6 & 5.088752 & 1.224124 & 11.963189 \\ 1 & 4.679988 & 0.320972 & 11.493797 \\ 1 & 5.562757 & 1.811700 & 11.157221 \\ 6 & -0.385710 & 1.298870 & 10.783941 \\ 6 & 1.836267 & -0.328347 & 10.852938 \\ 1 & 2.002843 & -1.250707 & 10.289919 \\ 1 & 1.564556 & -0.472240 & 11.897800 \\ 6 & 3.340090 & 1.211019 & 13.752252 \\ 1 & 2.553635 & 1.796729 & 14.257551 \\ 1 & 2.852138 & 0.331533 & 13.307106 \\ 6 & 5.048455 & 1.970857 & 15.414323 \\ 1 & 5.787475 & 1.655456 & 16.169394 \\ 1 & 4.289081 & 2.578753 & 15.934608 \\ 6 & 1.947167 & 3.479389 & 11.574330 \\ 6 & 1.093063 & 1.986684 & 8.948709 \\ 6 & 4.688652 & 3.255460 & 13.285535 \\ 1 & 5.193570 & 3.852522 & 12.512113 \\ 1 & 3.976225 & 3.903712 & 13.801236 \\ 6 & 0.634335 & 0.857814 & 8.182912 \\ 1 & 0.943018 & 0.760359 & 7.140787 \\ 6 & 5.454731 & -0.093133 & 14.056841 \\ 1 & 4.986419 & -0.976443 & 13.592309 \\ 1 & 6.199608 & -0.460353 & 14.782118 \\ 6 & 6.813008 & 1.966949 & 13.636626 \\ 1 & 7.569052 & 1.637029 & 14.368542 \\ 1 & 7.333298 & 2.571072 & 12.875418 \\ 6 & 0.354753 & 2.293080 & 10.165142 \\ 6 & 2.035688 & 4.896543 & 12.216164 \\ 6 & 1.797518 & 3.095622 & 8.152793 \\ 1 & 1.914274 & 3.951561 & 8.831021 \\ 6 & 1.637494 & 4.815939 & 13.713030 \\ 1 & 0.578047 & 4.523225 & 13.760150 \\ 1 & 2.175197 & 3.995024 & 14.204600 \\ 6 & -0.435580 & 0.510181 & 13.208682 \\ 1 & -0.543213 & -0.551369 & 12.936691 \\ 1 & -0.955832 & 0.659736 & 14.166807 \\ 1 & 0.629475 & 0.725698 & 13.365344 \\ 6 & -0.061322 & -0.130727 & 8.802339 \\ 1 & -0.329069 & -1.051902 & 8.281793\end{array}$




$\begin{array}{rrrr}6 & -0.062604 & 4.710819 & 10.872441 \\ 6 & 3.348502 & 5.691938 & 11.980225 \\ 1 & 3.074577 & 6.756971 & 12.064155 \\ 1 & 4.082109 & 5.530579 & 12.773333 \\ 6 & -2.547490 & 1.236136 & 12.053535 \\ 1 & -2.999109 & 1.888848 & 11.292769 \\ 1 & -3.031991 & 1.439967 & 13.020519 \\ 1 & -2.780301 & 0.196282 & 11.774989 \\ 6 & 0.951994 & 5.694058 & 11.448018 \\ 1 & 0.479243 & 6.446480 & 12.096319 \\ 1 & 1.422236 & 6.238828 & 10.616712 \\ 6 & -0.500905 & 5.126766 & 9.465885 \\ 1 & 0.355463 & 5.295859 & 8.802528 \\ 1 & -1.074487 & 6.063200 & 9.524852 \\ 1 & -1.150653 & 4.358126 & 9.021314 \\ 6 & 1.846805 & 6.101895 & 14.507013 \\ 1 & 1.345810 & 6.962167 & 14.037939 \\ 1 & 2.913451 & 6.350192 & 14.601819 \\ 1 & 1.440666 & 5.997044 & 15.522667 \\ 6 & -1.346240 & 4.639654 & 11.716830 \\ 1 & -2.030045 & 3.874337 & 11.324371 \\ 1 & -1.859493 & 5.610396 & 11.659156 \\ 1 & -1.152910 & 4.426688 & 12.775005 \\ 6 & 4.006274 & 5.457350 & 10.624346 \\ 1 & 3.307947 & 5.642828 & 9.792564 \\ 1 & 4.357024 & 4.420554 & 10.524902 \\ 1 & 4.864018 & 6.131362 & 10.489138 \\ 6 & 3.189455 & 2.732352 & 7.627901 \\ 1 & 3.930801 & 2.659757 & 8.432313 \\ 1 & 3.523737 & 3.501412 & 6.915110 \\ 1 & 3.174728 & 1.765687 & 7.099760 \\ 6 & 0.922520 & 3.516537 & 6.959843 \\ 1 & 1.004191 & 2.780179 & 6.146164 \\ 1 & 1.261829 & 4.484163 & 6.559864 \\ 1 & -0.138283 & 3.601076 & 7.226016\end{array}$

2:

$\begin{array}{cccc}15 & 2.713902 & 1.167203 & 10.160004 \\ 7 & 0.712612 & 3.474976 & 10.928450 \\ 6 & -1.047192 & 1.309654 & 12.101502 \\ 1 & -0.962732 & 2.331382 & 12.485695 \\ 6 & 4.271063 & 0.955711 & 14.874991 \\ 1 & 3.734914 & 0.518845 & 15.732547 \\ 6 & 5.833812 & 2.763133 & 14.137006 \\ 1 & 6.410818 & 3.644267 & 14.460506 \\ 6 & 5.939703 & 0.528036 & 13.065724\end{array}$




$\begin{array}{rrrc}1 & 6.594078 & -0.226522 & 12.601760 \\ 6 & 2.807899 & 2.413716 & 11.557238 \\ 6 & 3.927622 & 2.049812 & 12.582385 \\ 6 & 0.143960 & -0.104240 & 10.281417 \\ 1 & -0.468607 & -0.911380 & 10.705470 \\ 6 & 4.912169 & 0.987106 & 12.021728 \\ 1 & 4.361377 & 0.094564 & 11.686021 \\ 1 & 5.423879 & 1.387929 & 11.131858 \\ 6 & -0.253388 & 1.248739 & 10.819885 \\ 6 & 1.644655 & -0.277197 & 10.732905 \\ 1 & 2.052394 & -1.201686 & 10.297077 \\ 1 & 1.711858 & -0.358616 & 11.825517 \\ 6 & 3.248561 & 1.397143 & 13.817560 \\ 1 & 2.520626 & 2.098972 & 14.254559 \\ 1 & 2.673767 & 0.519919 & 13.480652 \\ 6 & 5.092355 & 2.164756 & 15.338623 \\ 1 & 5.810616 & 1.859529 & 16.117444 \\ 1 & 4.428621 & 2.923788 & 15.786859 \\ 6 & 1.950178 & 3.503750 & 11.582423 \\ 6 & 1.346952 & 1.961876 & 9.080592 \\ 6 & 4.804074 & 3.213475 & 13.090135 \\ 1 & 5.313552 & 3.691279 & 12.237784 \\ 1 & 4.174253 & 3.965825 & 13.568453 \\ 6 & 0.796259 & 0.877413 & 8.201053 \\ 1 & 0.944735 & 0.932568 & 7.122032 \\ 6 & 5.210107 & -0.090797 & 14.264491 \\ 1 & 4.631502 & -0.971882 & 13.941067 \\ 1 & 5.938755 & -0.435935 & 15.016475 \\ 6 & 6.775559 & 1.721030 & 13.533371 \\ 1 & 7.522957 & 1.398573 & 14.277217 \\ 1 & 7.324152 & 2.156668 & 12.682375 \\ 6 & 0.400290 & 2.279506 & 10.238835 \\ 6 & 2.062005 & 4.928913 & 12.206678 \\ 6 & 1.871329 & 3.164655 & 8.260389 \\ 1 & 1.945221 & 4.014361 & 8.953486 \\ 6 & 1.767854 & 4.878914 & 13.726845 \\ 1 & 0.746010 & 4.491428 & 13.861772 \\ 1 & 2.418521 & 4.137457 & 14.206760 \\ 6 & -0.503269 & 0.386104 & 13.196344 \\ 1 & -0.513952 & -0.671783 & 12.891251 \\ 1 & -1.124786 & 0.472962 & 14.100084 \\ 1 & 0.525629 & 0.661748 & 13.464575 \\ 6 & 0.142341 & -0.129094 & 8.780752 \\ 1 & -0.304397 & -0.955715 & 8.226012 \\ 6 & -0.093408 & 4.705992 & 10.952484 \\ 6 & 3.359272 & 5.723538 & 11.893118\end{array}$




$\begin{array}{rrrc}1 & 3.095257 & 6.787815 & 12.013737 \\ 1 & 4.142695 & 5.545280 & 12.635036 \\ 6 & -2.525345 & 1.010741 & 11.826104 \\ 1 & -2.940682 & 1.705371 & 11.081629 \\ 1 & -3.120347 & 1.088475 & 12.748631 \\ 1 & -2.648747 & -0.010427 & 11.431578 \\ 6 & 0.930738 & 5.705965 & 11.484299 \\ 1 & 0.471022 & 6.452439 & 12.147427 \\ 1 & 1.356485 & 6.255242 & 10.632400 \\ 6 & -0.596499 & 5.104957 & 9.565557 \\ 1 & 0.232131 & 5.294915 & 8.873350 \\ 1 & -1.194382 & 6.024748 & 9.643684 \\ 1 & -1.237884 & 4.315634 & 9.146022 \\ 6 & 1.918582 & 6.206275 & 14.465320 \\ 1 & 1.312243 & 7.006467 & 14.015693 \\ 1 & 2.963726 & 6.546821 & 14.475624 \\ 1 & 1.597656 & 6.099389 & 15.510894 \\ 6 & -1.326964 & 4.608367 & 11.864237 \\ 1 & -2.034069 & 3.856223 & 11.486223 \\ 1 & -1.842571 & 5.579713 & 11.875450 \\ 1 & -1.062015 & 4.352907 & 12.898278 \\ 6 & 3.935558 & 5.516747 & 10.496571 \\ 1 & 3.205945 & 5.763068 & 9.708853 \\ 1 & 4.240995 & 4.472670 & 10.341533 \\ 1 & 4.811145 & 6.163272 & 10.343163 \\ 6 & 3.265014 & 2.938884 & 7.668924 \\ 1 & 4.038085 & 2.850477 & 8.443664 \\ 1 & 3.530921 & 3.779654 & 7.010158 \\ 1 & 3.291893 & 2.015610 & 7.067373 \\ 6 & 0.908540 & 3.541417 & 7.124086 \\ 1 & 1.066878 & 2.896522 & 6.246946 \\ 1 & 1.084893 & 4.576856 & 6.795227 \\ 1 & -0.144718 & 3.446745 & 7.419652\end{array}$

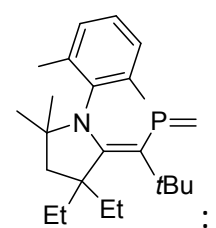

$\begin{array}{cccc}15 & -0.792544 & 2.056857 & -1.012103 \\ 7 & -0.571795 & -0.818178 & -0.088308 \\ 6 & 0.715463 & 1.237249 & -0.252815 \\ 6 & 1.830830 & 2.299482 & -0.058082 \\ 6 & 1.259032 & 3.456735 & 0.792330 \\ 1 & 0.385684 & 3.935643 & 0.328109 \\ 1 & 0.956385 & 3.095035 & 1.787363 \\ 6 & -0.606099 & 1.629591 & -2.606599\end{array}$




$\begin{array}{rrrr}1 & 0.194487 & 0.979377 & -2.976381 \\ 1 & -1.325081 & 1.996246 & -3.346703 \\ 6 & 2.282210 & 2.870749 & -1.418350 \\ 1 & 2.643233 & 2.067008 & -2.078757 \\ 1 & 1.474648 & 3.395609 & -1.943052 \\ 6 & 0.655317 & -0.103648 & 0.007155 \\ 6 & 3.105186 & 1.855762 & 0.662178 \\ 1 & 2.894693 & 1.424944 & 1.646890 \\ 1 & 3.694226 & 1.148067 & 0.068009 \\ 6 & 1.787906 & -1.173316 & 0.186555 \\ 6 & 2.584226 & -1.184420 & -1.146326 \\ 1 & 1.878501 & -1.314003 & -1.978239 \\ 1 & 3.005733 & -0.177891 & -1.286341 \\ 6 & -0.395060 & -2.280575 & -0.249979 \\ 6 & 2.802751 & -1.134727 & 1.360480 \\ 1 & 3.234085 & -2.149608 & 1.392874 \\ 1 & 3.649443 & -0.484878 & 1.120729 \\ 6 & 0.978901 & -2.467282 & 0.389683 \\ 1 & 1.508211 & -3.348261 & 0.000777 \\ 1 & 0.834276 & -2.628243 & 1.469197 \\ 6 & -1.458514 & -3.061685 & 0.519553 \\ 1 & -1.496977 & -2.742920 & 1.571329 \\ 1 & -1.214900 & -4.134008 & 0.484272 \\ 1 & -2.458257 & -2.922262 & 0.084412 \\ 6 & 3.700187 & -2.218554 & -1.265183 \\ 1 & 3.332909 & -3.245746 & -1.121235 \\ 1 & 4.504765 & -2.044996 & -0.537217 \\ 1 & 4.149715 & -2.171708 & -2.266994 \\ 6 & -0.380976 & -2.764431 & -1.715919 \\ 1 & -1.391117 & -2.967574 & -2.088358 \\ 1 & 0.191564 & -3.700942 & -1.790274 \\ 1 & 0.078888 & -2.024979 & -2.383999 \\ 6 & 2.294466 & -0.808476 & 2.758655 \\ 1 & 1.565371 & -1.547052 & 3.121489 \\ 1 & 1.817130 & 0.178707 & 2.809575 \\ 1 & 3.134661 & -0.812649 & 3.468054 \\ 1 & 3.736724 & 2.742996 & 0.819144 \\ 1 & 3.106595 & 3.583630 & -1.261822 \\ 1 & 2.028772 & 4.231817 & 0.929040 \\ 6 & -1.835846 & -0.264927 & 0.276971 \\ 6 & -2.921158 & -0.248133 & -0.629332 \\ 6 & -2.019694 & 0.252561 & 1.582585 \\ 6 & -4.142104 & 0.302280 & -0.225594 \\ 6 & -3.247976 & 0.811417 & 1.937019 \\ 6 & -4.311620 & 0.840437 & 1.042249 \\ 1 & -4.966946 & 0.323594 & -0.941016\end{array}$




$\begin{array}{rrrr}1 & -3.369851 & 1.214549 & 2.944523 \\ 1 & -5.267419 & 1.277961 & 1.334117 \\ 6 & -0.920617 & 0.211215 & 2.599850 \\ 1 & -0.365630 & -0.732828 & 2.532903 \\ 1 & -0.192432 & 1.017527 & 2.424352 \\ 1 & -1.324818 & 0.318049 & 3.614778 \\ 6 & -2.814240 & -0.762077 & -2.036893 \\ 1 & -3.156570 & -1.806742 & -2.107724 \\ 1 & -3.451737 & -0.163044 & -2.701658 \\ 1 & -1.783684 & -0.707400 & -2.403606\end{array}$

\begin{tabular}{|c|c|c|c|}
\hline J & 2.831801 & 2.361597 & 1.228307 \\
\hline & 2.543487 & 1.774627 & 2.118212 \\
\hline U & 2.627599 & 1.665800 & -1.214062 \\
\hline & 2.137084 & 2.617626 & -1.421694 \\
\hline O & 3.028674 & 1.373850 & 0.090014 \\
\hline & 3.857026 & -0.795919 & -0.699784 \\
\hline U & 3.438878 & -0.457582 & -1.987202 \\
\hline & 3.584188 & -1.170929 & -2.7 \\
\hline & 3.661029 & 0.135080 & 665 \\
\hline & 4.540956 & -2.1 & 934 \\
\hline 1 & 4.162958 & -2.46 & 2469 \\
\hline 6 & 1.708899 & & \\
\hline 1 & 1.967641 & 4.0 & 719 \\
\hline & 1.533034 & 3.9 & \\
\hline 1 & 0.770060 & 2.86 & 680 \\
\hline & 2.831392 & 0.76 & 685 \\
\hline 1 & 2.509283 & 1.0 & 3020 \\
\hline & 4.142359 & 3.1 & 325 \\
\hline 1 & 4.978415 & 2.4 & 028 \\
\hline & 4.035140 & 3.7 & 006 \\
\hline 1 & 4.423462 & 3.72 & 7875 \\
\hline & 4.218947 & -3.219694 & 5349 \\
\hline 1 & 3.134422 & -3.337998 & -1.549112 \\
\hline 1 & 4.632327 & -4.179588 & -1.073379 \\
\hline 1 & 4.664361 & -3.004003 & 8214 \\
\hline 6 & 6.062558 & -1.9 & 219 \\
\hline 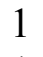 & 6.464674 & -1.6 & -1.277859 \\
\hline 1 & 6.558733 & -2.870433 & -0.000575 \\
\hline 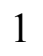 & 6.338123 & -1.150662 & 0.420495 \\
\hline 1 & 4.028132 & -0.198736 & 1.648700 \\
\hline & 4.733031 & -0.914879 & 1.750153 \\
\hline & 4.160732 & 0.568973 & 2.291367 \\
\hline
\end{tabular}




\section{TS-S1:}

$\begin{array}{cccc}15 & 0.551337 & 0.386768 & -0.552515 \\ 7 & -2.340568 & 0.341371 & -0.549255 \\ 6 & 2.767088 & 2.333710 & 1.250183 \\ 1 & 2.645051 & 1.786195 & 2.197570 \\ 6 & -0.478808 & -0.776435 & 0.540456 \\ 6 & 0.226676 & -1.560524 & 1.657141 \\ 6 & 2.997903 & 1.798658 & -1.218227 \\ 1 & 2.804747 & 2.855623 & -1.413292 \\ 6 & 0.785839 & -0.503925 & 2.629951 \\ 1 & 1.419210 & 0.216402 & 2.102264 \\ 1 & -0.037443 & 0.043483 & 3.115247 \\ 6 & 2.932225 & 1.311412 & 0.131968 \\ 6 & 1.237367 & -0.767144 & -1.696395 \\ 1 & 1.084245 & -1.837782 & -1.514959 \\ 1 & 1.104667 & -0.498675 & -2.746176 \\ 6 & 1.384420 & -2.403634 & 1.109315 \\ 1 & 1.011873 & -3.192837 & 0.436413 \\ 1 & 2.100221 & -1.785537 & 0.559172 \\ 6 & -1.807726 & -0.745100 & 0.202350 \\ 6 & 3.835224 & -0.819921 & -0.634386 \\ 6 & -0.640928 & -2.494061 & 2.500092 \\ 1 & -1.536005 & -1.989276 & 2.881489 \\ 1 & -0.933822 & -3.394242 & 1.946853 \\ 6 & 3.231827 & -0.485220 & -1.893228 \\ 1 & 3.408136 & -1.179293 & -2.717090 \\ 6 & 3.651264 & 0.088924 & 0.394241 \\ 6 & -2.862102 & -1.918668 & 0.142166 \\ 6 & 4.528298 & -2.149187 & -0.393094 \\ 1 & 4.214580 & -2.515262 & 0.601599 \\ 6 & -2.166113 & -3.035390 & -0.688487 \\ 1 & -1.777098 & -2.591661 & -1.614982 \\ 1 & -1.272461 & -3.346762 & -0.125550 \\ 6 & 1.537190 & 3.227638 & 1.096147 \\ 1 & 1.545261 & 3.772904 & 0.139973 \\ 1 & 1.508859 & 3.974617 & 1.903675 \\ 1 & 0.604070 & 2.647520 & 1.129575 \\ 6 & 3.169477 & 0.919195 & -2.236256 \\ 1 & 3.119235 & 1.229267 & -3.281597 \\ 6 & -3.451167 & -0.076685 & -1.433768 \\ 6 & -3.526604 & -2.612015 & 1.367301 \\ 1 & -4.359073 & -3.187022 & 0.927884 \\ 1 & -2.858089 & -3.372421 & 1.784640 \\ 6 & 4.044970 & 3.181005 & 1.360780 \\ 1 & 4.945238 & 2.554795 & 1.461082\end{array}$




$\begin{array}{rrrr}1 & 3.994124 & 3.859435 & 2.225491 \\ 1 & 4.175548 & 3.793227 & 0.455676 \\ 6 & -4.013254 & -1.255807 & -0.636868 \\ 1 & -4.544535 & -1.976470 & -1.274476 \\ 1 & -4.747059 & -0.860334 & 0.082304 \\ 6 & -4.507304 & 1.015132 & -1.585106 \\ 1 & -4.860721 & 1.361168 & -0.603028 \\ 1 & -5.362844 & 0.610574 & -2.146390 \\ 1 & -4.116561 & 1.884354 & -2.131463 \\ 6 & -2.998844 & -4.267433 & -1.033709 \\ 1 & -3.930537 & -4.006944 & -1.558513 \\ 1 & -3.267232 & -4.854447 & -0.144553 \\ 1 & -2.426184 & -4.928519 & -1.699552 \\ 6 & -2.976487 & -0.529584 & -2.831781 \\ 1 & -2.947693 & 0.309638 & -3.537282 \\ 1 & -3.663961 & -1.284628 & -3.241818 \\ 1 & -1.967493 & -0.962688 & -2.786849 \\ 6 & -4.118093 & -1.790758 & 2.506641 \\ 1 & -4.892534 & -1.090537 & 2.162560 \\ 1 & -3.365382 & -1.210566 & 3.053850 \\ 1 & -4.598607 & -2.469541 & 3.226196 \\ 6 & 4.138050 & -3.238107 & -1.390800 \\ 1 & 3.046347 & -3.358174 & -1.449670 \\ 1 & 4.572709 & -4.201840 & -1.088622 \\ 1 & 4.513758 & -3.011218 & -2.400006 \\ 6 & 6.052790 & -1.965570 & -0.372766 \\ 1 & 6.403372 & -1.631926 & -1.360913 \\ 1 & 6.565866 & -2.907202 & -0.123480 \\ 1 & 6.358550 & -1.199656 & 0.355742 \\ 1 & -0.049684 & -2.827156 & 3.366837 \\ 1 & 1.920418 & -2.889605 & 1.940224 \\ 1 & 1.398118 & -0.983805 & 3.409293 \\ 6 & -2.119949 & 1.717417 & -0.227100 \\ 6 & -1.671690 & 2.637008 & -1.202593 \\ 6 & -2.395897 & 2.183001 & 1.078800 \\ 6 & -1.513818 & 3.982246 & -0.854146 \\ 6 & -2.204819 & 3.531369 & 1.391948 \\ 6 & -1.770334 & 4.437936 & 0.432613 \\ 1 & -1.159467 & 4.679008 & -1.617551 \\ 1 & -2.428042 & 3.870235 & 2.405875 \\ 1 & -1.632239 & 5.490282 & 0.685381 \\ 6 & -2.942137 & 1.263513 & 2.128185 \\ 1 & -3.734228 & 0.635293 & 1.702788 \\ 1 & -2.168879 & 0.579351 & 2.507328 \\ 1 & -3.355700 & 1.831821 & 2.971641 \\ 6 & -1.327188 & 2.233862 & -2.610619\end{array}$




$\begin{array}{rrrr}1 & -2.157834 & 2.441326 & -3.305740 \\ 1 & -0.455569 & 2.807901 & -2.955697 \\ 1 & -1.077564 & 1.170461 & -2.670993 \\ 7 & 4.028755 & -0.209854 & 1.704873 \\ 1 & 4.694079 & -0.965160 & 1.800322 \\ 1 & 4.274815 & 0.583009 & 2.284017\end{array}$

$\begin{array}{lccc}\text { P-S1: } & & \\ 15 & 0.677544 & 0.382115 & -0.437445 \\ 7 & -2.219752 & 0.420159 & -0.471215 \\ 6 & 2.482322 & 2.188055 & 1.135691 \\ 1 & 2.412344 & 1.719789 & 2.128269 \\ 6 & -0.382231 & -0.701870 & 0.695894 \\ 6 & 0.227284 & -1.537360 & 1.838903 \\ 6 & 2.767935 & 1.631661 & -1.306952 \\ 1 & 2.721142 & 2.711016 & -1.463018 \\ 6 & 0.829245 & -0.528052 & 2.833552 \\ 1 & 1.642224 & 0.031457 & 2.367672 \\ 1 & 0.061776 & 0.175698 & 3.192481 \\ 6 & 2.480531 & 1.066286 & 0.071813 \\ 6 & 1.364867 & -0.944111 & -1.591491 \\ 1 & 1.221212 & -1.947565 & -1.169977 \\ 1 & 0.777281 & -0.884041 & -2.521643 \\ 6 & 1.338304 & -2.491385 & 1.374227 \\ 1 & 0.937242 & -3.272983 & 0.709513 \\ 1 & 2.140707 & -1.963892 & 0.849542 \\ 6 & -1.695598 & -0.670641 & 0.271612 \\ 6 & 3.679870 & -0.946301 & -0.661853 \\ 6 & -0.757585 & -2.381636 & 2.645864 \\ 1 & -1.648035 & -1.811628 & 2.936036 \\ 1 & -1.057514 & -3.282919 & 2.101692 \\ 6 & 2.871158 & -0.687013 & -1.913955 \\ 1 & 3.182766 & -1.344885 & -2.735128 \\ 6 & 3.461255 & -0.063332 & 0.336317 \\ 6 & -2.733619 & -1.861736 & 0.128260 \\ 6 & 4.576983 & -2.157322 & -0.511013 \\ 1 & 4.519830 & -2.481000 & 0.545230 \\ 6 & -1.965210 & -2.963305 & -0.657202 \\ 1 & -1.519425 & -2.506867 & -1.552084 \\ 1 & -1.107678 & -3.267133 & -0.037450 \\ 6 & 1.289631 & 3.132445 & 1.018368 \\ 1 & 1.226962 & 3.579077 & 0.014099 \\ 1 & 1.380828 & 3.952638 & 1.746397 \\ 1 & 0.334893 & 2.619011 & 1.202560 \\ 6 & 2.986984 & 0.769285 & -2.300218 \\ 1 & 3.165031 & 1.078737 & -3.331654\end{array}$




$\begin{array}{rrrr}6 & -3.250451 & 0.002031 & -1.447838 \\ 6 & -3.497567 & -2.575310 & 1.285920 \\ 1 & -4.277515 & -3.150622 & 0.759393 \\ 1 & -2.869662 & -3.336931 & 1.756816 \\ 6 & 3.792833 & 2.989440 & 1.094134 \\ 1 & 4.682444 & 2.343376 & 1.045276 \\ 1 & 3.876913 & 3.631107 & 1.983930 \\ 1 & 3.824008 & 3.647029 & 0.213411 \\ 6 & -3.836552 & -1.211190 & -0.727394 \\ 1 & -4.289035 & -1.932598 & -1.422317 \\ 1 & -4.638951 & -0.855337 & -0.063901 \\ 6 & -4.322976 & 1.070923 & -1.643903 \\ 1 & -4.747537 & 1.383548 & -0.678792 \\ 1 & -5.129451 & 0.656248 & -2.266852 \\ 1 & -3.924028 & 1.962731 & -2.145394 \\ 6 & -2.751230 & -4.205558 & -1.068837 \\ 1 & -3.651468 & -3.956676 & -1.651427 \\ 1 & -3.065808 & -4.806873 & -0.204807 \\ 1 & -2.123595 & -4.848573 & -1.701879 \\ 6 & -2.673348 & -0.408057 & -2.819866 \\ 1 & -2.588638 & 0.447628 & -3.499237 \\ 1 & -3.332247 & -1.148058 & -3.298191 \\ 1 & -1.674164 & -0.853122 & -2.714416 \\ 6 & -4.211716 & -1.780770 & 2.373732 \\ 1 & -4.950246 & -1.076310 & 1.965611 \\ 1 & -3.528429 & -1.210530 & 3.014227 \\ 1 & -4.762838 & -2.479083 & 3.020212 \\ 6 & 4.124583 & -3.358066 & -1.345130 \\ 1 & 3.063239 & -3.590382 & -1.171989 \\ 1 & 4.715885 & -4.248538 & -1.086139 \\ 1 & 4.263696 & -3.175230 & -2.421085 \\ 6 & 6.038504 & -1.806058 & -0.820171 \\ 1 & 6.132377 & -1.469130 & -1.864150 \\ 1 & 6.702996 & -2.672696 & -0.677689 \\ 1 & 6.392754 & -0.985481 & -0.178477 \\ 1 & -0.257138 & -2.717058 & 3.566480 \\ 1 & 1.779798 & -2.991426 & 2.250491 \\ 1 & 1.253626 & -1.057660 & 3.700964 \\ 6 & -2.039340 & 1.794776 & -0.124255 \\ 6 & -1.582904 & 2.744447 & -1.065442 \\ 6 & -2.403163 & 2.231291 & 1.171370 \\ 6 & -1.515463 & 4.092457 & -0.696072 \\ 6 & -2.293681 & 3.582616 & 1.507928 \\ 6 & -1.859072 & 4.521029 & 0.579301 \\ 1 & -1.158087 & 4.814875 & -1.433979 \\ 1 & -2.584379 & 3.897904 & 2.512246\end{array}$




$\begin{array}{rrrr}1 & -1.789447 & 5.576311 & 0.847238 \\ 6 & -2.967009 & 1.273325 & 2.176501 \\ 1 & -3.739661 & 0.654167 & 1.704816 \\ 1 & -2.199682 & 0.583325 & 2.557853 \\ 1 & -3.413593 & 1.809084 & 3.024180 \\ 6 & -1.149882 & 2.384096 & -2.460314 \\ 1 & -1.955486 & 2.560248 & -3.192490 \\ 1 & -0.295079 & 3.009402 & -2.755159 \\ 1 & -0.833784 & 1.339098 & -2.517782 \\ 7 & 3.976626 & -0.215979 & 1.625805 \\ 1 & 4.775662 & -0.834149 & 1.690218 \\ 1 & 4.130281 & 0.640337 & 2.142797\end{array}$

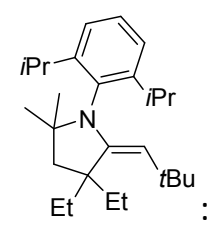

$\begin{array}{llll}7 & 0.755001 & 3.734652 & 10.929168\end{array}$

$\begin{array}{llll}6 & -1.018356 & 1.777106 & 12.292089\end{array}$

$\begin{array}{llll}1 & -0.595918 & 2.708204 & 12.691046\end{array}$

$\begin{array}{llll}6 & 2.607502 & 2.590760 & 11.932271\end{array}$

$\begin{array}{llll}6 & 3.829045 & 2.135058 & 12.726338\end{array}$

$\begin{array}{llll}6 & -1.096812 & 0.606731 & 10.073417\end{array}$

$\begin{array}{llll}1 & -1.809773 & -0.086432 & 10.525824\end{array}$

$\begin{array}{llll}6 & 4.605400 & 1.150010 & 11.833148\end{array}$

$\begin{array}{llll}1 & 3.944666 & 0.356421 & 11.451904\end{array}$

$\begin{array}{lllll}1 & 5.045005 & 1.670792 & 10.968689\end{array}$

$\begin{array}{llll}6 & -0.615811 & 1.682028 & 10.829001\end{array}$

$\begin{array}{llll}6 & 3.337413 & 1.366625 & 13.967799\end{array}$

$\begin{array}{llll}1 & 2.727014 & 2.014607 & 14.614976\end{array}$

$\begin{array}{llll}1 & 2.722141 & 0.501992 & 13.678669\end{array}$

$\begin{array}{llll}6 & 1.918697 & 3.737272 & 11.713168\end{array}$

$\begin{array}{llll}6 & 0.785075 & 2.331275 & 8.925569\end{array}$

$\begin{array}{llll}6 & 4.830633 & 3.193778 & 13.191652\end{array}$

$\begin{array}{llll}1 & 5.250528 & 3.752546 & 12.344683\end{array}$

$\begin{array}{llll}1 & 4.395456 & 3.902365 & 13.908301\end{array}$

$\begin{array}{llll}6 & 0.281245 & 1.241838 & 8.210794\end{array}$

$\begin{array}{lllll}1 & 0.648614 & 1.049589 & 7.200395\end{array}$

$\begin{array}{llll}6 & 0.296069 & 2.581105 & 10.229820\end{array}$

$\begin{array}{llll}6 & 2.120072 & 5.174072 & 12.224530\end{array}$

$\begin{array}{llll}6 & 1.897287 & 3.161987 & 8.307607\end{array}$

$\begin{array}{lllll}1 & 2.072420 & 4.021546 & 8.967250\end{array}$

$\begin{array}{llll}6 & 1.834092 & 5.189680 & 13.744958\end{array}$

$\begin{array}{lllll}1 & 0.790549 & 4.869772 & 13.898393\end{array}$

$\begin{array}{lllll}1 & 2.449663 & 4.413775 & 14.223524\end{array}$

$\begin{array}{llll}6 & -0.400366 & 0.619552 & 13.085028\end{array}$ 


\begin{tabular}{|c|c|c|c|}
\hline & 130 & 48641 & 12.733998 \\
\hline & -0.638840 & 0.714504 & \\
\hline & 0.691141 & 0.611462 & 12.976854 \\
\hline & -0.668818 & 0.393396 & 0908 \\
\hline & -1.057807 & -0.448784 & 194313 \\
\hline & 0.018718 & 9517 & \\
\hline & 3.495533 & 5.808283 & 11.912553 \\
\hline & 3.371305 & 6.899600 & 12.015736 \\
\hline & 4.230304 & 5.528824 & 12.675231 \\
\hline & -2.536778 & 1.788655 & 12.494984 \\
\hline & -3.035319 & 2.557981 & $11.8901 \mathrm{G}$ \\
\hline & -2.778686 & 1.972500 & 13.552510 \\
\hline & -2.976785 & 0.816967 & 12.223443 \\
\hline & 1.051557 & 5.985771 & 11.452679 \\
\hline & 0.577242 & 6.7 & 6356 \\
\hline & 1.528588 & 6.530461 & 10.625083 \\
\hline & -0.359773 & 5.391631 & 9.442562 \\
\hline & 0.531101 & 5.484765 & 8.807374 \\
\hline & -0.879672 & 6.3 & 880 \\
\hline & -1.032823 & 4.6 & 23 \\
\hline & 2.071535 & 6.526614 & 14.440344 \\
\hline & 1.515 & & \\
\hline & 3.136018 & & \\
\hline & 1.745 & 6.4 & 556 \\
\hline & -1.269672 & 4.98 & 11.709290 \\
\hline & -1.964271 & 4.231970 & 11.320901 \\
\hline & -1.766217 & 5.9 & 11.645140 \\
\hline & -1.07 & $4.7^{\prime}$ & 12.7 \\
\hline & 4.048507 & 5.496749 & 10.526552 \\
\hline & 3.367183 & 5.836927 & 9.730459 \\
\hline & 4.194202 & 4.414587 & 10.391516 \\
\hline & 5.01 & & 10.36 \\
\hline & $3.1 \mathrm{C}$ & 2.3 & 8.2 \\
\hline & 3.465404 & 1.992425 & 9.268768 \\
\hline & 4.026122 & 2.971746 & 7.886413 \\
\hline & 3.095363 & 1.479265 & 7.601486 \\
\hline & 1.54 & 3.679482 & 6.911430 \\
\hline & 1.466090 & 2.85 & 6.18 \\
\hline & 2.323525 & 4.362704 & 6.546602 \\
\hline & 0.582954 & 4.216819 & 6.907724 \\
\hline & 5.665 & 2.688849 & 13.702361 \\
\hline & 4.190596 & 0.990035 & 14.554879 \\
\hline & 5.4224 & 0.673135 & 12.396902 \\
\hline & 2.142597 & 1.721047 & 11.45289 \\
\hline
\end{tabular}




$\begin{array}{cccc}15 & 2.516432 & -3.610356 & 11.613618 \\ 6 & 3.083358 & -3.176765 & 10.117286 \\ 1 & 3.967552 & -2.536768 & 10.045757 \\ 1 & 2.634931 & -3.485815 & 9.167241 \\ 6 & 1.042760 & -4.666124 & 11.209304 \\ 1 & 0.145667 & -4.225949 & 11.668894 \\ 1 & 0.882198 & -4.770720 & 10.128157 \\ 1 & 1.177826 & -5.662656 & 11.654865\end{array}$

\section{TS-S2:}

$\begin{array}{crcc}15 & 1.193082 & -0.585808 & 10.912602 \\ 7 & 0.839508 & 3.588580 & 10.954527 \\ 6 & -0.936622 & 1.638221 & 12.276451 \\ 1 & -0.677135 & 2.673458 & 12.519042 \\ 6 & 2.787829 & 2.583504 & 11.914162 \\ 6 & 4.035846 & 2.172590 & 12.686983 \\ 6 & -1.253471 & 0.716095 & 9.896948 \\ 1 & -2.185123 & 0.258307 & 10.231787 \\ 6 & 4.918361 & 1.361454 & 11.720036 \\ 1 & 4.341360 & 0.544308 & 11.257842 \\ 1 & 5.313644 & 2.004379 & 10.918290 \\ 6 & -0.439199 & 1.426782 & 10.838528 \\ 6 & 1.657288 & -0.458238 & 9.249008 \\ 1 & 2.624607 & 0.005052 & 9.048576 \\ 1 & 1.406180 & -1.274301 & 8.564022 \\ 6 & 3.581786 & 1.227083 & 13.815654 \\ 1 & 2.942005 & 1.759068 & 14.536442 \\ 1 & 3.008652 & 0.382716 & 13.402389 \\ 6 & 2.014160 & 3.676863 & 11.723176 \\ 6 & 0.965415 & 2.170012 & 8.984051 \\ 6 & 4.914094 & 3.263493 & 13.299535 \\ 1 & 5.339249 & 3.922453 & 12.531026 \\ 1 & 4.374615 & 3.873894 & 14.036190 \\ 6 & 0.505142 & 0.979661 & 8.307562 \\ 1 & 0.868936 & 0.828807 & 7.287651 \\ 6 & 0.469452 & 2.409380 & 10.246740 \\ 6 & 2.109602 & 5.116127 & 12.247653 \\ 6 & 2.020257 & 3.035165 & 8.319473 \\ 1 & 2.165674 & 3.909672 & 8.967684 \\ 6 & 1.746809 & 5.102158 & 13.752260 \\ 1 & 0.725869 & 4.699651 & 13.854323 \\ 1 & 2.398300 & 4.372480 & 14.255914 \\ 6 & -0.248359 & 0.798207 & 13.356702 \\ 1 & -0.450590 & -0.276412 & 13.231411 \\ 1 & -0.631419 & 1.097009 & 14.344784 \\ 1 & 0.836864 & 0.951006 & 13.346898\end{array}$




$\begin{array}{cccc}6 & -0.813943 & 0.526987 & 8.617232 \\ 1 & -1.375332 & -0.079758 & 7.904863 \\ 6 & 0.057761 & 4.837424 & 10.841198 \\ 6 & 3.462195 & 5.824673 & 12.014123 \\ 1 & 3.273159 & 6.907257 & 12.110253 \\ 1 & 4.165320 & 5.582916 & 12.819572 \\ 6 & -2.454726 & 1.491694 & 12.410266 \\ 1 & -2.999769 & 2.056835 & 11.640166 \\ 1 & -2.779875 & 1.854324 & 13.396359 \\ 1 & -2.763991 & 0.437273 & 12.334850 \\ 6 & 1.038258 & 5.866029 & 11.421793 \\ 1 & 0.517284 & 6.623290 & 12.025502 \\ 1 & 1.524303 & 6.402003 & 10.593498 \\ 6 & -0.291408 & 5.176482 & 9.388975 \\ 1 & 0.612198 & 5.305520 & 8.779608 \\ 1 & -0.855205 & 6.120463 & 9.361342 \\ 1 & -0.913748 & 4.390208 & 8.935274 \\ 6 & 1.847641 & 6.447609 & 14.463619 \\ 1 & 1.245205 & 7.224066 & 13.968710 \\ 1 & 2.885535 & 6.807214 & 14.504785 \\ 1 & 1.487996 & 6.362719 & 15.498542 \\ 6 & -1.262017 & 4.811121 & 11.628878 \\ 1 & -1.923493 & 4.014351 & 11.258627 \\ 1 & -1.782241 & 5.771067 & 11.495847 \\ 1 & -1.100975 & 4.662532 & 12.705163 \\ 6 & 4.112458 & 5.551594 & 10.661827 \\ 1 & 3.450887 & 5.835179 & 9.827740 \\ 1 & 4.348789 & 4.484269 & 10.540372 \\ 1 & 5.042081 & 6.128313 & 10.552822 \\ 6 & 3.377221 & 2.328223 & 8.226400 \\ 1 & 3.716321 & 1.994367 & 9.217117 \\ 1 & 4.131360 & 3.019977 & 7.821180 \\ 1 & 3.333816 & 1.457205 & 7.552744 \\ 6 & 1.584114 & 3.513732 & 6.930110 \\ 1 & 1.547744 & 2.675502 & 6.217437 \\ 1 & 2.302450 & 4.247948 & 6.535039 \\ 1 & 0.588990 & 3.978777 & 6.950725 \\ 1 & 5.754270 & 2.784309 & 13.825632 \\ 1 & 4.452007 & 0.820370 & 14.355887 \\ 1 & 5.772913 & 0.915315 & 12.252843 \\ 6 & -0.061022 & -1.959200 & 10.796706 \\ 1 & -0.797800 & -1.886574 & 11.609458 \\ 1 & -0.588352 & -1.946352 & 9.833018 \\ 1 & 0.465292 & -2.919797 & 10.900649 \\ 1 & 2.375020 & 1.691554 & 11.432381\end{array}$




\section{P-S2:}

$\begin{array}{cccc}15 & 0.786458 & -0.314569 & 10.893137 \\ 7 & 0.805991 & 3.479478 & 11.081221 \\ 6 & -1.193421 & 1.483791 & 12.032562 \\ 1 & -1.561984 & 2.511323 & 11.945202 \\ 6 & 2.835662 & 2.544721 & 11.965841 \\ 6 & 4.090530 & 2.160288 & 12.739673 \\ 6 & -1.332630 & 0.877628 & 9.541785 \\ 1 & -2.399560 & 0.711976 & 9.691633 \\ 6 & 4.917464 & 1.230745 & 11.832639 \\ 1 & 4.290575 & 0.417932 & 11.433142 \\ 1 & 5.343616 & 1.788009 & 10.984082 \\ 6 & -0.437643 & 1.207555 & 10.710886 \\ 6 & 1.360512 & -0.292290 & 9.114237 \\ 1 & 2.455354 & -0.194693 & 9.101107 \\ 1 & 1.097778 & -1.244376 & 8.629410 \\ 6 & 3.609264 & 1.339468 & 13.952837 \\ 1 & 3.032258 & 1.973147 & 14.643714 \\ 1 & 2.957934 & 0.514109 & 13.626255 \\ 6 & 2.051115 & 3.621588 & 11.749971 \\ 6 & 1.098356 & 2.179216 & 9.059550 \\ 6 & 5.025268 & 3.260752 & 13.241756 \\ 1 & 5.433683 & 3.858119 & 12.415775 \\ 1 & 4.536018 & 3.931069 & 13.960563 \\ 6 & 0.734703 & 0.903362 & 8.328157 \\ 1 & 1.136711 & 0.907122 & 7.306184 \\ 6 & 0.519475 & 2.344522 & 10.267179 \\ 6 & 2.184688 & 5.088045 & 12.181823 \\ 6 & 2.108215 & 3.108181 & 8.432798 \\ 1 & 2.197228 & 3.983332 & 9.093416 \\ 6 & 1.866772 & 5.173077 & 13.694581 \\ 1 & 0.898423 & 4.686729 & 13.882938 \\ 1 & 2.608438 & 4.552912 & 14.220865 \\ 6 & -0.300359 & 1.427493 & 13.274468 \\ 1 & -0.061694 & 0.384958 & 13.541280 \\ 1 & -0.825065 & 1.875953 & 14.132442 \\ 1 & 0.649156 & 1.959069 & 13.131916 \\ 6 & -0.761174 & 0.742187 & 8.341373 \\ 1 & -1.314139 & 0.475663 & 7.439309 \\ 6 & 0.028451 & 4.740685 & 10.999937 \\ 6 & 3.540605 & 5.772170 & 11.893396 \\ 1 & 3.357399 & 6.859747 & 11.924358 \\ 1 & 4.241413 & 5.574835 & 12.713492 \\ 6 & -2.422801 & 0.597448 & 12.260166 \\ 1 & -3.227410 & 0.781097 & 11.536410 \\ 1 & -2.836070 & 0.801196 & 13.258869\end{array}$




$\begin{array}{rrrc}1 & -2.170065 & -0.471624 & 12.222357 \\ 6 & 1.116010 & 5.775003 & 11.313522 \\ 1 & 0.711029 & 6.681452 & 11.784039 \\ 1 & 1.581471 & 6.085061 & 10.365008 \\ 6 & -0.558933 & 4.971758 & 9.608835 \\ 1 & 0.229471 & 5.025621 & 8.847591 \\ 1 & -1.111007 & 5.923588 & 9.603376 \\ 1 & -1.254894 & 4.166006 & 9.330845 \\ 6 & 1.863969 & 6.573371 & 14.299627 \\ 1 & 1.121689 & 7.230667 & 13.822911 \\ 1 & 2.846645 & 7.058767 & 14.213969 \\ 1 & 1.615107 & 6.524771 & 15.369071 \\ 6 & -1.130041 & 4.838622 & 12.016894 \\ 1 & -2.039522 & 4.354674 & 11.634905 \\ 1 & -1.379366 & 5.896098 & 12.190377 \\ 1 & -0.875911 & 4.377301 & 12.979510 \\ 6 & 4.201184 & 5.424343 & 10.563325 \\ 1 & 3.578771 & 5.720582 & 9.704534 \\ 1 & 4.385430 & 4.343476 & 10.478086 \\ 1 & 5.162446 & 5.948771 & 10.462794 \\ 6 & 3.486269 & 2.441600 & 8.357088 \\ 1 & 3.811121 & 2.105780 & 9.351628 \\ 1 & 4.234257 & 3.148402 & 7.965988 \\ 1 & 3.468661 & 1.570300 & 7.681856 \\ 6 & 1.673650 & 3.582820 & 7.040559 \\ 1 & 1.707979 & 2.759281 & 6.311190 \\ 1 & 2.353421 & 4.368050 & 6.676956 \\ 1 & 0.650165 & 3.983579 & 7.042158 \\ 1 & 5.873926 & 2.790621 & 13.762682 \\ 1 & 4.465508 & 0.913532 & 14.500989 \\ 1 & 5.749555 & 0.778083 & 12.394281 \\ 6 & -0.316415 & -1.809631 & 10.825654 \\ 1 & -0.756197 & -2.007149 & 11.812318 \\ 1 & -1.110014 & -1.728416 & 10.068709 \\ 1 & 0.325307 & -2.667536 & 10.575804 \\ 1 & 2.395716 & 1.623693 & 11.573080\end{array}$

\section{$\mathbf{S M}(\mathbf{A s}=\mathbf{C})$ :}

$\begin{array}{llll}7 & 0.821118 & 3.162204 & 11.168814\end{array}$

$\begin{array}{llll}6 & -1.372633 & 1.179445 & 11.761645\end{array}$

$\begin{array}{llll}1 & -0.926801 & 1.907522 & 12.451661\end{array}$

$\begin{array}{lllll}6 & 2.971002 & 2.310940 & 12.006856\end{array}$

$\begin{array}{lllll}6 & 4.387626 & 2.241446 & 12.660267\end{array}$

$\begin{array}{llll}6 & -0.783055 & 0.170914 & 9.560274\end{array}$

$\begin{array}{lllll}1 & -1.559886 & -0.565689 & 9.773693\end{array}$

$\begin{array}{llll}6 & 5.348001 & 1.401105 & 11.792292\end{array}$ 


$\begin{array}{rrrc}1 & 4.984331 & 0.382085 & 11.624477 \\ 1 & 5.513235 & 1.881737 & 10.815806 \\ 6 & -0.526479 & 1.176928 & 10.496524 \\ 6 & 2.829379 & -0.438022 & 10.677438 \\ 1 & 3.524629 & -0.066887 & 9.921043 \\ 1 & 2.507356 & -1.478172 & 10.575449 \\ 6 & 4.264138 & 1.555983 & 14.040962 \\ 1 & 3.563819 & 2.103895 & 14.689829 \\ 1 & 3.915881 & 0.515562 & 13.968818 \\ 6 & 2.124355 & 3.351405 & 11.735242 \\ 6 & 1.169146 & 2.080372 & 8.982152 \\ 6 & 5.156676 & 3.545169 & 12.911722 \\ 1 & 5.368421 & 4.084463 & 11.982679 \\ 1 & 4.662309 & 4.212988 & 13.623452 \\ 6 & 0.910739 & 1.027727 & 8.103053 \\ 1 & 1.468207 & 0.972200 & 7.165304 \\ 6 & 0.489729 & 2.132032 & 10.222562 \\ 6 & 2.223708 & 4.867065 & 12.122659 \\ 6 & 2.193089 & 3.113083 & 8.550694 \\ 1 & 2.214817 & 3.894654 & 9.317748 \\ 6 & 2.062521 & 4.957287 & 13.664011 \\ 1 & 1.111885 & 4.488171 & 13.946529 \\ 1 & 2.832879 & 4.328019 & 14.129263 \\ 6 & -1.411504 & -0.174407 & 12.481259 \\ 1 & -1.908752 & -0.941891 & 11.869652 \\ 1 & -1.987978 & -0.079229 & 13.413752 \\ 1 & -0.406414 & -0.531721 & 12.736132 \\ 6 & -0.052768 & 0.067693 & 8.385197 \\ 1 & -0.246695 & -0.744050 & 7.682313 \\ 6 & -0.037157 & 4.375872 & 11.235373 \\ 6 & 3.432875 & 5.732494 & 11.677707 \\ 1 & 3.068997 & 6.773778 & 11.692995 \\ 1 & 4.226867 & 5.711330 & 12.427579 \\ 6 & -2.815665 & 1.595646 & 11.436850 \\ 1 & -2.868459 & 2.518331 & 10.843700 \\ 1 & -3.395415 & 1.744315 & 12.360939 \\ 1 & -3.312302 & 0.805312 & 10.852877 \\ 6 & 1.009608 & 5.470278 & 11.401604 \\ 1 & 0.616984 & 6.352636 & 11.926321 \\ 1 & 1.323584 & 5.803569 & 10.399667 \\ 6 & -0.820431 & 4.602555 & 9.942552 \\ 1 & -0.145855 & 4.656194 & 9.077907 \\ 1 & -1.363819 & 5.556035 & 10.020132 \\ 1 & -1.550026 & 3.803276 & 9.756451 \\ 6 & 2.129043 & 6.360359 & 14.261066 \\ 1 & 1.383032 & 7.037036 & 13.817878\end{array}$




$\begin{array}{cccc}1 & 3.118433 & 6.819930 & 14.125163 \\ 1 & 1.931549 & 6.320772 & 15.341398 \\ 6 & -1.035923 & 4.374802 & 12.411401 \\ 1 & -1.944557 & 3.813822 & 12.167728 \\ 1 & -1.339483 & 5.408203 & 12.636575 \\ 1 & -0.606554 & 3.935146 & 13.319198 \\ 6 & 4.026148 & 5.451411 & 10.303221 \\ 1 & 3.323911 & 5.688409 & 9.489916 \\ 1 & 4.312268 & 4.395542 & 10.198354 \\ 1 & 4.923360 & 6.067021 & 10.144030 \\ 6 & 3.594793 & 2.505472 & 8.455879 \\ 1 & 3.906677 & 2.098136 & 9.426698 \\ 1 & 4.327479 & 3.265304 & 8.142940 \\ 1 & 3.618573 & 1.691534 & 7.714202 \\ 6 & 1.815858 & 3.770622 & 7.218085 \\ 1 & 1.882602 & 3.052013 & 6.387278 \\ 1 & 2.507300 & 4.597419 & 6.994162 \\ 1 & 0.791428 & 4.169012 & 7.228992 \\ 1 & 6.126760 & 3.274786 & 13.354487 \\ 1 & 5.248691 & 1.535014 & 14.532963 \\ 1 & 6.323535 & 1.330951 & 12.297330 \\ 33 & 2.136748 & 0.499931 & 12.010641\end{array}$

\begin{tabular}{|c|c|c|c|}
\hline \multicolumn{4}{|c|}{$S(A s=C):$} \\
\hline & 8347 & 3.338456 & 11.0111 \\
\hline & -1.046012 & 1.222609 & 11.969250 \\
\hline & -0.674097 & 2.084370 & 12.538479 \\
\hline 6 & 2.823097 & 2.267699 & 6412 \\
\hline 5 & 4.152035 & 2.077373 & 12.722073 \\
\hline 5 & -0.502333 & 0.018319 & 9.837 \\
\hline & -1.244219 & -0.720036 & 10.146277 \\
\hline & 5.118626 & 1.153033 & 11.948063 \\
\hline & 4.733213 & & \\
\hline 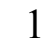 & 5.32 & 1.5 & \\
\hline & -0.170604 & 1.0 & 10 \\
\hline ) & 2.711191 & -0.029011 & 9.881744 \\
\hline 1 & 3.551692 & 8160 & 0673 \\
\hline & 2.63 & -1.0 & \\
\hline & 3.83 & 1.4 & \\
\hline & 3.180763 & 2.06 & 14. \\
\hline & 3.327263 & 0.44 & 4806 \\
\hline 6 & 2.060008 & 3.386924 & 11.707972 \\
\hline f & & & \\
\hline f & 4.99 & & \\
\hline & 5.31 & 3.83 & 12.125153 \\
\hline 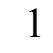 & 4.491855 & 4.024285 & 13.702490 \\
\hline
\end{tabular}




$\begin{array}{rrrc}6 & 1.222977 & 0.798097 & 8.382125 \\ 1 & 1.789476 & 0.661200 & 7.457585 \\ 6 & 0.531898 & 2.227561 & 10.173553 \\ 6 & 2.211438 & 4.871040 & 12.189165 \\ 6 & 2.015763 & 3.197041 & 8.326385 \\ 1 & 1.968642 & 4.065033 & 8.998158 \\ 6 & 1.988747 & 4.913976 & 13.723100 \\ 1 & 0.978091 & 4.542051 & 13.938829 \\ 1 & 2.661006 & 4.188293 & 14.199253 \\ 6 & -1.032830 & 0.004166 & 12.895906 \\ 1 & -1.287471 & -0.917640 & 12.350161 \\ 1 & -1.784729 & 0.135883 & 13.688132 \\ 1 & -0.053880 & -0.144088 & 13.367413 \\ 6 & 0.189172 & -0.132980 & 8.674143 \\ 1 & 0.030009 & -0.996219 & 8.025311 \\ 6 & -0.001626 & 4.554522 & 11.092405 \\ 6 & 3.486300 & 5.669087 & 11.804013 \\ 1 & 3.197523 & 6.732492 & 11.856189 \\ 1 & 4.271503 & 5.559540 & 12.555260 \\ 6 & -2.493091 & 1.492028 & 11.523860 \\ 1 & -2.547238 & 2.246874 & 10.726708 \\ 1 & -3.106822 & 1.830405 & 12.372450 \\ 1 & -2.947270 & 0.569948 & 11.130190 \\ 6 & 1.061424 & 5.588961 & 11.446756 \\ 1 & 0.657608 & 6.422618 & 12.039268 \\ 1 & 1.451901 & 6.017516 & 10.511043 \\ 6 & -0.673049 & 4.870036 & 9.755878 \\ 1 & 0.066357 & 5.004519 & 8.956161 \\ 1 & -1.253469 & 5.799429 & 9.849200 \\ 1 & -1.360464 & 4.064026 & 9.458053 \\ 6 & 2.174306 & 6.279477 & 14.379595 \\ 1 & 1.544563 & 7.051875 & 13.913041 \\ 1 & 3.217230 & 6.622412 & 14.324554 \\ 1 & 1.900097 & 6.230958 & 15.442751 \\ 6 & -1.105142 & 4.511308 & 12.165923 \\ 1 & -1.929486 & 3.854270 & 11.869059 \\ 1 & -1.518878 & 5.522400 & 12.296263 \\ 1 & -0.730407 & 4.168908 & 13.137729 \\ 6 & 4.061956 & 5.387179 & 10.423241 \\ 1 & 3.357803 & 5.646181 & 9.617936 \\ 1 & 4.314146 & 4.324563 & 10.307500 \\ 1 & 4.974814 & 5.977603 & 10.258054 \\ 6 & 3.492127 & 2.816861 & 8.181157 \\ 1 & 3.925241 & 2.543832 & 9.153309 \\ 1 & 4.061924 & 3.667808 & 7.777798 \\ 1 & 3.625664 & 1.970385 & 7.489142\end{array}$




$\begin{array}{cccc}6 & 1.438010 & 3.601046 & 6.964710 \\ 1 & 1.512986 & 2.767889 & 6.248599 \\ 1 & 1.991045 & 4.454834 & 6.544189 \\ 1 & 0.376459 & 3.875579 & 7.041843 \\ 1 & 5.903665 & 2.984898 & 13.559427 \\ 1 & 4.763349 & 1.242699 & 14.643274 \\ 1 & 6.071667 & 1.082428 & 12.493614 \\ 33 & 2.086941 & 0.419059 & 11.549015\end{array}$

\section{$\mathbf{P}(\mathbf{A s}=\mathbf{C})$ :}

$\begin{array}{llll}7 & 0.873015 & 3.423794 & 10.917333\end{array}$

$\begin{array}{lllll}6 & -0.807325 & 1.230952 & 12.028766\end{array}$

$\begin{array}{llll}1 & -0.475921 & 2.136622 & 12.554988\end{array}$

$\begin{array}{llll}6 & 2.731261 & 2.262597 & 11.975833\end{array}$

$\begin{array}{llll}6 & 4.010347 & 2.011336 & 12.825812\end{array}$

$\begin{array}{llll}6 & -0.318094 & -0.030894 & 9.900372\end{array}$

$\begin{array}{lllll}1 & -1.163274 & -0.662260 & 10.180286\end{array}$

$\begin{array}{llll}6 & 4.953386 & 1.055399 & 12.060177\end{array}$

$\begin{array}{lllll}1 & 4.512235 & 0.064793 & 11.878060\end{array}$

$\begin{array}{llll}1 & 5.239840 & 1.491145 & 11.089638\end{array}$

$\begin{array}{lllll}6 & 0.159067 & 1.021635 & 10.850592\end{array}$

$\begin{array}{lllll}6 & 2.618110 & 0.205167 & 9.563505\end{array}$

$\begin{array}{lllll}1 & 3.557610 & 0.753009 & 9.421318\end{array}$

$\begin{array}{lllll}1 & 2.816954 & -0.866020 & 9.413871\end{array}$

$\begin{array}{llll}6 & 3.611166 & 1.348236 & 14.161524\end{array}$

$\begin{array}{lllll}1 & 2.972683 & 2.023061 & 14.751268\end{array}$

$\begin{array}{lllll}1 & 3.059827 & 0.410777 & 14.006264\end{array}$

$\begin{array}{lllll}6 & 2.021798 & 3.414151 & 11.728430\end{array}$

$\begin{array}{llll}6 & 1.243519 & 2.123895 & 8.930078\end{array}$

$\begin{array}{llll}6 & 4.901220 & 3.207677 & 13.184348\end{array}$

$\begin{array}{lllll}1 & 5.288972 & 3.714879 & 12.292422\end{array}$

$\begin{array}{lllll}1 & 4.404564 & 3.936568 & 13.831310\end{array}$

$\begin{array}{llll}6 & 1.524391 & 0.691552 & 8.543944\end{array}$

$\begin{array}{lllll}1 & 1.918473 & 0.616277 & 7.520799\end{array}$

$\begin{array}{lllll}6 & 0.626680 & 2.272755 & 10.125086\end{array}$

$\begin{array}{lllll}6 & 2.191422 & 4.881040 & 12.239037\end{array}$

$\begin{array}{llll}6 & 1.909474 & 3.230823 & 8.150677\end{array}$

$\begin{array}{lllll}1 & 1.843734 & 4.138312 & 8.767530\end{array}$

$\begin{array}{llll}6 & 1.942044 & 4.928712 & 13.766724\end{array}$

$\begin{array}{lllll}1 & 0.901342 & 4.630911 & 13.956591\end{array}$

$\begin{array}{lllll}1 & 2.550262 & 4.156381 & 14.255808\end{array}$

$\begin{array}{llll}6 & -0.790938 & 0.082210 & 13.039549\end{array}$

$\begin{array}{lllll}1 & -0.965177 & -0.886933 & 12.544945\end{array}$

$\begin{array}{lllll}1 & -1.586184 & 0.223824 & 13.786678\end{array}$

$\begin{array}{lllll}1 & 0.167704 & 0.015443 & 13.572178\end{array}$

$\begin{array}{llll}6 & 0.308927 & -0.169143 & 8.730791\end{array}$ 


$\begin{array}{cccc}1 & 0.012850 & -0.901167 & 7.977742 \\ 6 & 0.024237 & 4.621889 & 11.035828 \\ 6 & 3.506448 & 5.616121 & 11.864154 \\ 1 & 3.271261 & 6.693300 & 11.895384 \\ 1 & 4.278208 & 5.476438 & 12.625290 \\ 6 & -2.239581 & 1.443760 & 11.520094 \\ 1 & -2.266041 & 2.097604 & 10.635207 \\ 1 & -2.874153 & 1.885920 & 12.302590 \\ 1 & -2.697925 & 0.485943 & 11.232431 \\ 6 & 1.073423 & 5.638913 & 11.480626 \\ 1 & 0.644740 & 6.443485 & 12.095665 \\ 1 & 1.499903 & 6.108410 & 10.581804 \\ 6 & -0.612002 & 4.989726 & 9.696241 \\ 1 & 0.142993 & 5.225445 & 8.935426 \\ 1 & -1.256104 & 5.871642 & 9.823922 \\ 1 & -1.233451 & 4.161514 & 9.322548 \\ 6 & 2.207017 & 6.278282 & 14.428733 \\ 1 & 1.649874 & 7.091887 & 13.939925 \\ 1 & 3.273287 & 6.544370 & 14.402305 \\ 1 & 1.899230 & 6.254755 & 15.483438 \\ 6 & -1.111045 & 4.519471 & 12.070552 \\ 1 & -1.906547 & 3.851987 & 11.723102 \\ 1 & -1.552077 & 5.516948 & 12.215622 \\ 1 & -0.760222 & 4.159041 & 13.045088 \\ 6 & 4.073761 & 5.270146 & 10.495211 \\ 1 & 3.356325 & 5.476421 & 9.686132 \\ 1 & 4.327879 & 4.202715 & 10.437873 \\ 1 & 4.981711 & 5.854569 & 10.287969 \\ 6 & 3.396415 & 2.973439 & 7.884507 \\ 1 & 3.945645 & 2.810949 & 8.821753 \\ 1 & 3.842935 & 3.841970 & 7.376981 \\ 1 & 3.550235 & 2.097170 & 7.235676 \\ 6 & 1.164291 & 3.473794 & 6.832937 \\ 1 & 1.193536 & 2.573393 & 6.198775 \\ 1 & 1.626391 & 4.297518 & 6.267729 \\ 1 & 0.108594 & 3.720466 & 7.013588 \\ 1 & 5.767870 & 2.825537 & 13.744061 \\ 1 & 4.510024 & 1.117747 & 14.755581 \\ 1 & 5.871685 & 0.890696 & 12.643718 \\ 33 & 2.017853 & 0.451300 & 11.452521\end{array}$

\section{$\mathbf{S M}(\mathbf{S i}=\mathbf{C})$ :}

$\begin{array}{llll}7 & 0.881295 & 3.358393 & 10.893369\end{array}$

$\begin{array}{llll}6 & -0.939206 & 1.250368 & 11.990671\end{array}$

$\begin{array}{lllll}1 & -0.579445 & 2.159530 & 12.483613\end{array}$

$\begin{array}{llll}6 & 2.874595 & 2.272226 & 11.843284\end{array}$ 


$\begin{array}{rrrc}6 & 4.021167 & 2.062288 & 12.901581 \\ 6 & -0.654835 & 0.145409 & 9.755508 \\ 1 & -1.267350 & -0.669436 & 10.147486 \\ 6 & 5.062281 & 1.035181 & 12.398836 \\ 1 & 4.649158 & 0.027336 & 12.259274 \\ 1 & 5.496130 & 1.344068 & 11.436079 \\ 6 & -0.327897 & 1.216984 & 10.596485 \\ 6 & 3.376576 & 1.469530 & 14.172966 \\ 1 & 2.648817 & 2.162688 & 14.618017 \\ 1 & 2.848233 & 0.531143 & 13.948988 \\ 6 & 2.068122 & 3.373262 & 11.649529 \\ 6 & 0.904352 & 2.236439 & 8.724192 \\ 6 & 4.879328 & 3.264348 & 13.323392 \\ 1 & 5.397903 & 3.709912 & 12.464055 \\ 1 & 4.323663 & 4.041146 & 13.852670 \\ 6 & 0.544506 & 1.150414 & 7.929362 \\ 1 & 0.884188 & 1.109463 & 6.892472 \\ 6 & 0.481102 & 2.260071 & 10.077748 \\ 6 & 2.218704 & 4.841239 & 12.165978 \\ 6 & 1.778254 & 3.327456 & 8.126411 \\ 1 & 1.809254 & 4.149064 & 8.851556 \\ 6 & 1.935957 & 4.923303 & 13.687900 \\ 1 & 0.878177 & 4.681578 & 13.855454 \\ 1 & 2.491966 & 4.139804 & 14.213875 \\ 6 & -0.558266 & 0.070546 & 12.892182 \\ 1 & -0.692059 & -0.894529 & 12.379974 \\ 1 & -1.202958 & 0.069171 & 13.783878 \\ 1 & 0.482210 & 0.135530 & 13.230298 \\ 6 & -0.215901 & 0.102828 & 8.440855 \\ 1 & -0.471757 & -0.747503 & 7.806820 \\ 6 & 0.051320 & 4.584888 & 10.986770 \\ 6 & 3.542166 & 5.568025 & 11.791212 \\ 1 & 3.298279 & 6.643605 & 11.783226 \\ 1 & 4.300536 & 5.461729 & 12.569161 \\ 6 & -2.470274 & 1.318148 & 11.871629 \\ 1 & -2.797976 & 2.050723 & 11.120527 \\ 1 & -2.921957 & 1.582103 & 12.839829 \\ 1 & -2.875217 & 0.339442 & 11.570623 \\ 6 & 1.113724 & 5.596485 & 11.394728 \\ 1 & 0.701695 & 6.426034 & 11.987738 \\ 1 & 1.542691 & 6.031989 & 10.479238 \\ 6 & -0.609146 & 4.968467 & 9.660144 \\ 1 & 0.123698 & 5.219302 & 8.884968 \\ 1 & -1.238123 & 5.855142 & 9.824545 \\ 1 & -1.255081 & 4.159415 & 9.287017 \\ 6 & 2.243615 & 6.273328 & 14.331035\end{array}$




$\begin{array}{crrc}1 & 1.736569 & 7.101386 & 13.812967 \\ 1 & 3.320859 & 6.491335 & 14.328689 \\ 1 & 1.907340 & 6.282964 & 15.377131 \\ 6 & -1.083751 & 4.492206 & 12.022865 \\ 1 & -1.877138 & 3.820156 & 11.674182 \\ 1 & -1.527225 & 5.489908 & 12.156290 \\ 1 & -0.742889 & 4.137303 & 13.001892 \\ 6 & 4.147691 & 5.187890 & 10.446890 \\ 1 & 3.434589 & 5.325096 & 9.619336 \\ 1 & 4.459838 & 4.134151 & 10.435997 \\ 1 & 5.026235 & 5.812043 & 10.229689 \\ 6 & 3.225535 & 2.870772 & 7.922496 \\ 1 & 3.683485 & 2.562980 & 8.872182 \\ 1 & 3.821964 & 3.695768 & 7.502755 \\ 1 & 3.276608 & 2.020565 & 7.224760 \\ 6 & 1.201969 & 3.860325 & 6.810350 \\ 1 & 1.288599 & 3.112174 & 6.008052 \\ 1 & 1.758427 & 4.752424 & 6.485869 \\ 1 & 0.139813 & 4.127109 & 6.905572 \\ 1 & 5.649851 & 2.900929 & 14.019452 \\ 1 & 4.150003 & 1.247661 & 14.926008 \\ 1 & 5.871768 & 0.949736 & 13.138672 \\ 1 & 3.037327 & -1.187700 & 9.475784 \\ 14 & 2.564773 & 0.595709 & 11.010756 \\ 1 & 2.028493 & -0.420174 & 11.948822 \\ 6 & 3.213859 & -0.115145 & 9.599731 \\ 1 & 3.716893 & 0.379406 & 8.771340\end{array}$

\section{$\mathbf{T S}(\mathbf{S i}=\mathbf{C})$ :}

$\begin{array}{rrrc}7 & 0.882926 & 3.447708 & 10.834412 \\ 6 & -0.751071 & 1.280282 & 12.039041 \\ 1 & -0.400639 & 2.211995 & 12.496963 \\ 6 & 2.738147 & 2.261705 & 11.889869 \\ 6 & 3.915159 & 1.987085 & 12.882233 \\ 6 & -0.478425 & 0.089013 & 9.837866 \\ 1 & -1.134260 & -0.681320 & 10.249199 \\ 6 & 4.903015 & 0.965228 & 12.275035 \\ 1 & 4.451910 & -0.019673 & 12.094527 \\ 1 & 5.302151 & 1.325794 & 11.314759 \\ 6 & -0.051060 & 1.156939 & 10.682945 \\ 6 & 3.323400 & 1.362388 & 14.162764 \\ 1 & 2.628330 & 2.054990 & 14.659561 \\ 1 & 2.772747 & 0.437748 & 13.937549 \\ 6 & 2.016499 & 3.411922 & 11.668947 \\ 6 & 1.016647 & 2.218700 & 8.743100 \\ 6 & 4.811178 & 3.157980 & 13.309669\end{array}$




$\begin{array}{cccc}1 & 5.313823 & 3.617213 & 12.448089 \\ 1 & 4.282037 & 3.930859 & 13.872635 \\ 6 & 0.948244 & 0.941702 & 8.127042 \\ 1 & 1.395174 & 0.821067 & 7.137349 \\ 6 & 0.535060 & 2.319121 & 10.044122 \\ 6 & 2.187952 & 4.865874 & 12.205075 \\ 6 & 1.775275 & 3.331760 & 8.046264 \\ 1 & 1.776710 & 4.190760 & 8.728319 \\ 6 & 1.902042 & 4.918634 & 13.726825 \\ 1 & 0.839738 & 4.686784 & 13.885509 \\ 1 & 2.446351 & 4.113243 & 14.234129 \\ 6 & -0.465178 & 0.151894 & 13.033783 \\ 1 & -0.580100 & -0.838662 & 12.566332 \\ 1 & -1.176232 & 0.211818 & 13.871176 \\ 1 & 0.547476 & 0.219117 & 13.448476 \\ 6 & 0.007195 & -0.017809 & 8.565272 \\ 1 & -0.231747 & -0.878219 & 7.938695 \\ 6 & 0.046313 & 4.659978 & 10.958870 \\ 6 & 3.529379 & 5.564664 & 11.847830 \\ 1 & 3.320810 & 6.647455 & 11.866609 \\ 1 & 4.286915 & 5.411599 & 12.619973 \\ 6 & -2.270458 & 1.369201 & 11.814837 \\ 1 & -2.527183 & 2.004347 & 10.955479 \\ 1 & -2.772861 & 1.765748 & 12.710153 \\ 1 & -2.687064 & 0.369622 & 11.616886 \\ 6 & 1.098882 & 5.654397 & 11.437717 \\ 1 & 0.668860 & 6.455028 & 12.057149 \\ 1 & 1.548529 & 6.130982 & 10.553908 \\ 6 & -0.575290 & 5.078291 & 9.625270 \\ 1 & 0.181393 & 5.363869 & 8.884610 \\ 1 & -1.229715 & 5.946287 & 9.789432 \\ 1 & -1.187004 & 4.264718 & 9.206196 \\ 6 & 2.227634 & 6.249712 & 14.399082 \\ 1 & 1.732326 & 7.094643 & 13.897090 \\ 1 & 3.307924 & 6.452355 & 14.400648 \\ 1 & 1.891371 & 6.243044 & 15.445208 \\ 6 & -1.110450 & 4.532634 & 11.966281 \\ 1 & -1.883928 & 3.852185 & 11.591651 \\ 1 & -1.573098 & 5.520934 & 12.105184 \\ 1 & -0.782571 & 4.171720 & 12.948131 \\ 6 & 4.109976 & 5.190900 & 10.490908 \\ 1 & 3.388821 & 5.356915 & 9.675489 \\ 1 & 4.390549 & 4.128464 & 10.463247 \\ 1 & 5.003616 & 5.791709 & 10.269781 \\ 6 & 3.243034 & 2.984233 & 7.779273 \\ 1 & 3.769454 & 2.743077 & 8.712942\end{array}$




$\begin{array}{cccc}1 & 3.749256 & 3.842505 & 7.311345 \\ 1 & 3.340978 & 2.125739 & 7.096608 \\ 6 & 1.075745 & 3.738870 & 6.745179 \\ 1 & 1.099897 & 2.914278 & 6.015979 \\ 1 & 1.578657 & 4.603813 & 6.286592 \\ 1 & 0.022022 & 3.999740 & 6.918076 \\ 1 & 5.594697 & 2.761958 & 13.973035 \\ 1 & 4.127061 & 1.107957 & 14.872363 \\ 1 & 5.743257 & 0.814204 & 12.968995 \\ 1 & 2.818158 & -1.009100 & 9.291545 \\ 14 & 2.244864 & 0.635782 & 11.032805 \\ 1 & 2.012215 & -0.479302 & 11.982323 \\ 6 & 2.838807 & 0.068274 & 9.481325 \\ 1 & 3.498846 & 0.635681 & 8.829244\end{array}$

\begin{tabular}{lccc}
\multicolumn{4}{l}{$\mathbf{P}(\mathbf{S i}=\mathbf{C}):$} \\
7 & 0.856625 & 3.477603 & 10.875197 \\
6 & -0.811519 & 1.272141 & 12.032834 \\
1 & -0.469775 & 2.188170 & 12.533317 \\
6 & 2.683738 & 2.280092 & 11.927494 \\
6 & 3.945064 & 1.982171 & 12.783481 \\
6 & -0.421990 & 0.050824 & 9.846605 \\
1 & -1.279986 & -0.561330 & 10.132135 \\
6 & 4.843683 & 0.990784 & 12.010689 \\
1 & 4.343918 & 0.037167 & 11.785513 \\
1 & 5.177816 & 1.435312 & 11.059815 \\
6 & 0.098013 & 1.068202 & 10.811238 \\
6 & 3.505303 & 1.322783 & 14.106855 \\
1 & 2.884611 & 2.014618 & 14.695321 \\
1 & 2.918511 & 0.410386 & 13.933937 \\
6 & 1.992550 & 3.449684 & 11.702834 \\
6 & 1.185642 & 2.169923 & 8.880598 \\
6 & 4.882763 & 3.138186 & 13.153430 \\
1 & 5.273267 & 3.650601 & 12.264983 \\
1 & 4.418589 & 3.872160 & 13.820171 \\
6 & 1.412829 & 0.733118 & 8.443650 \\
1 & 1.754585 & 0.682922 & 7.400513 \\
6 & 0.581419 & 2.325299 & 10.086481 \\
6 & 2.168155 & 4.901862 & 12.234321 \\
6 & 1.864767 & 3.273535 & 8.108032 \\
1 & 1.820861 & 4.175881 & 8.733517 \\
6 & 1.925964 & 4.924935 & 13.762845 \\
1 & 0.889196 & 4.612395 & 13.954349 \\
1 & 2.548164 & 4.152535 & 14.234525 \\
6 & -0.696240 & 0.131388 & 13.046405 \\
1 & -0.871052 & -0.844168 & 12.564131 \\
& & &
\end{tabular}




$\begin{array}{cccc}1 & -1.443804 & 0.248630 & 13.845246 \\ 1 & 0.297108 & 0.100161 & 13.516014 \\ 6 & 0.164211 & -0.079700 & 8.653462 \\ 1 & -0.185268 & -0.779964 & 7.893232 \\ 6 & 0.012563 & 4.678624 & 11.003299 \\ 6 & 3.497953 & 5.613744 & 11.871614 \\ 1 & 3.292178 & 6.695715 & 11.932537 \\ 1 & 4.267889 & 5.429911 & 12.625921 \\ 6 & -2.279402 & 1.446259 & 11.619816 \\ 1 & -2.376506 & 2.070310 & 10.718521 \\ 1 & -2.867472 & 1.903888 & 12.429734 \\ 1 & -2.740313 & 0.472897 & 11.393874 \\ 6 & 1.058033 & 5.683050 & 11.487213 \\ 1 & 0.619898 & 6.467909 & 12.120565 \\ 1 & 1.493062 & 6.180415 & 10.607889 \\ 6 & -0.601184 & 5.074778 & 9.661218 \\ 1 & 0.166309 & 5.326405 & 8.917953 \\ 1 & -1.246929 & 5.954372 & 9.795969 \\ 1 & -1.217213 & 4.254734 & 9.261198 \\ 6 & 2.183820 & 6.266785 & 14.442874 \\ 1 & 1.615563 & 7.082645 & 13.971098 \\ 1 & 3.247593 & 6.542521 & 14.412389 \\ 1 & 1.884774 & 6.224634 & 15.499503 \\ 6 & -1.137368 & 4.556762 & 12.018153 \\ 1 & -1.914779 & 3.874007 & 11.658690 \\ 1 & -1.597617 & 5.546633 & 12.155381 \\ 1 & -0.794152 & 4.204097 & 12.998605 \\ 6 & 4.049894 & 5.288023 & 10.490906 \\ 1 & 3.334835 & 5.535758 & 9.691163 \\ 1 & 4.275366 & 4.215963 & 10.403430 \\ 1 & 4.972114 & 5.853423 & 10.294448 \\ 6 & 3.344769 & 2.985522 & 7.834860 \\ 1 & 3.890218 & 2.794823 & 8.769698 \\ 1 & 3.810670 & 3.850771 & 7.339372 \\ 1 & 3.475021 & 2.114325 & 7.174139 \\ 6 & 1.116356 & 3.543198 & 6.797600 \\ 1 & 1.127019 & 2.651314 & 6.151366 \\ 1 & 1.590369 & 4.365927 & 6.241060 \\ 1 & 0.066390 & 3.807218 & 6.986880 \\ 1 & 5.744645 & 2.718877 & 13.694434 \\ 1 & 4.384695 & 1.048438 & 14.711661 \\ 1 & 5.736347 & 0.744968 & 12.605736 \\ 1 & 2.612990 & -0.899778 & 9.322410 \\ 14 & 1.959499 & 0.684187 & 11.176081 \\ 1 & 2.147279 & -0.482774 & 12.085298 \\ 6 & 2.518472 & 0.193036 & 9.417801\end{array}$


$\begin{array}{llll}1 & 3.493129 & 0.637824 & 9.174354\end{array}$

\begin{tabular}{lccc}
\multicolumn{4}{l}{$\mathbf{S M}(\mathbf{G e}=\mathbf{C}) \mathbf{:}$} \\
7 & 0.819008 & 3.175021 & 11.175800 \\
6 & -1.372152 & 1.134311 & 11.726871 \\
1 & -0.922914 & 1.816161 & 12.462012 \\
6 & 2.998954 & 2.370578 & 11.967589 \\
6 & 4.427596 & 2.294867 & 12.585417 \\
6 & -0.757942 & 0.186451 & 9.514678 \\
1 & -1.531702 & -0.557293 & 9.711791 \\
6 & 5.359234 & 1.515824 & 11.633439 \\
1 & 4.948457 & 0.539423 & 11.343139 \\
1 & 5.555403 & 2.101344 & 10.722773 \\
6 & -0.506715 & 1.172257 & 10.475386 \\
6 & 2.776527 & -0.854303 & 10.877236 \\
1 & 3.513551 & -0.785073 & 10.075938 \\
1 & 2.272755 & -1.813290 & 11.008335 \\
6 & 4.328859 & 1.519471 & 13.918572 \\
1 & 3.643181 & 2.025622 & 14.615384 \\
1 & 3.977366 & 0.484926 & 13.780682 \\
6 & 2.120549 & 3.389696 & 11.736766 \\
6 & 1.195388 & 2.104608 & 8.983127 \\
6 & 5.195418 & 3.583567 & 12.907692 \\
1 & 5.398263 & 4.178367 & 12.011251 \\
1 & 4.704714 & 4.207162 & 13.662591 \\
6 & 0.931172 & 1.077852 & 8.078011 \\
1 & 1.490556 & 1.043207 & 7.140941 \\
6 & 0.510196 & 2.137436 & 10.227571 \\
6 & 2.190879 & 4.893979 & 12.144705 \\
6 & 2.237098 & 3.128900 & 8.577209 \\
1 & 2.290077 & 3.881130 & 9.370460 \\
6 & 2.039014 & 4.957932 & 13.687958 \\
1 & 1.118833 & 4.435908 & 13.978421 \\
1 & 2.849187 & 4.363433 & 14.132105 \\
6 & -1.462789 & -0.262104 & 12.360934 \\
1 & -2.087634 & -0.933314 & 11.753771 \\
1 & -1.934716 & -0.189561 & 13.352128 \\
1 & -0.480465 & -0.733902 & 12.481361 \\
6 & -0.033257 & 0.113287 & 8.335988 \\
1 & -0.227029 & -0.682139 & 7.615044 \\
6 & -0.058845 & 4.374794 & 11.251218 \\
6 & 3.398071 & 5.768369 & 11.717072 \\
1 & 3.031707 & 6.808556 & 11.743560 \\
1 & 4.184969 & 5.735966 & 12.474918 \\
6 & -2.803626 & 1.593591 & 11.404728 \\
1 & -2.837499 & 2.551015 & 10.870057
\end{tabular}




$\begin{array}{rrrc}1 & -3.397693 & 1.690142 & 12.326672 \\ 1 & -3.297838 & 0.847946 & 10.763235 \\ 6 & 0.971388 & 5.485121 & 11.423993 \\ 1 & 0.563075 & 6.360613 & 11.947833 \\ 1 & 1.286051 & 5.823263 & 10.423708 \\ 6 & -0.845804 & 4.595432 & 9.960491 \\ 1 & -0.169955 & 4.659685 & 9.097851 \\ 1 & -1.401822 & 5.541365 & 10.039769 \\ 1 & -1.563835 & 3.787294 & 9.768769 \\ 6 & 2.044697 & 6.355449 & 14.300532 \\ 1 & 1.256160 & 6.997521 & 13.880490 \\ 1 & 3.006563 & 6.866148 & 14.151315 \\ 1 & 1.871280 & 6.293625 & 15.383906 \\ 6 & -1.047662 & 4.348202 & 12.433907 \\ 1 & -1.941167 & 3.760320 & 12.201651 \\ 1 & -1.379569 & 5.372743 & 12.659447 \\ 1 & -0.597012 & 3.924142 & 13.338587 \\ 6 & 4.004034 & 5.508340 & 10.343429 \\ 1 & 3.301891 & 5.740504 & 9.528592 \\ 1 & 4.310824 & 4.458702 & 10.229813 \\ 1 & 4.890611 & 6.141822 & 10.195599 \\ 6 & 3.615295 & 2.475227 & 8.442571 \\ 1 & 3.881383 & 1.929956 & 9.357284 \\ 1 & 4.389929 & 3.231935 & 8.243113 \\ 1 & 3.624697 & 1.758991 & 7.606584 \\ 6 & 1.868939 & 3.846897 & 7.273723 \\ 1 & 1.877134 & 3.152550 & 6.420151 \\ 1 & 2.602702 & 4.639424 & 7.060623 \\ 1 & 0.870602 & 4.304423 & 7.318677 \\ 1 & 6.170866 & 3.289868 & 13.323596 \\ 1 & 5.320850 & 1.456138 & 14.391209 \\ 1 & 6.325433 & 1.335166 & 12.129432 \\ 1 & 1.212151 & 0.378474 & 12.943860 \\ 32 & 2.253340 & 0.558548 & 11.821935\end{array}$

\section{$\mathbf{T S}(\mathbf{G e}=\mathbf{C})$ :

$\begin{array}{cccc}7 & 0.910751 & 3.403197 & 10.871728 \\ 6 & -0.764818 & 1.250545 & 12.055106 \\ 1 & -0.413663 & 2.180053 & 12.517136 \\ 6 & 2.793241 & 2.275791 & 11.963126 \\ 6 & 3.969584 & 2.038902 & 12.961398 \\ 6 & -0.467611 & 0.054239 & 9.859500 \\ 1 & -1.140729 & -0.707444 & 10.259155 \\ 6 & 4.977468 & 1.027676 & 12.368491 \\ 1 & 4.553701 & 0.024178 & 12.223099 \\ 1 & 5.355790 & 1.373327 & 11.394428\end{array}$




$\begin{array}{rrrc}6 & -0.050167 & 1.121635 & 10.708627 \\ 6 & 2.887065 & -0.028280 & 9.470183 \\ 1 & 3.545129 & 0.556082 & 8.832712 \\ 1 & 2.870319 & -1.103700 & 9.275900 \\ 6 & 3.384845 & 1.422665 & 14.249519 \\ 1 & 2.693650 & 2.120547 & 14.744114 \\ 1 & 2.831120 & 0.496688 & 14.036927 \\ 6 & 2.048562 & 3.400340 & 11.706760 \\ 6 & 1.071037 & 2.161415 & 8.786985 \\ 6 & 4.844790 & 3.230049 & 13.377519 \\ 1 & 5.347723 & 3.683196 & 12.512992 \\ 1 & 4.299706 & 4.003456 & 13.924248 \\ 6 & 0.999391 & 0.884200 & 8.169115 \\ 1 & 1.448826 & 0.762907 & 7.180789 \\ 6 & 0.571364 & 2.270253 & 10.081003 \\ 6 & 2.201226 & 4.868418 & 12.212932 \\ 6 & 1.843838 & 3.269483 & 8.097365 \\ 1 & 1.835508 & 4.131739 & 8.775685 \\ 6 & 1.903594 & 4.948494 & 13.731284 \\ 1 & 0.842749 & 4.709849 & 13.887315 \\ 1 & 2.451333 & 4.157552 & 14.257388 \\ 6 & -0.494477 & 0.120060 & 13.052279 \\ 1 & -0.614754 & -0.869538 & 12.583905 \\ 1 & -1.209149 & 0.185090 & 13.886193 \\ 1 & 0.516561 & 0.177937 & 13.473306 \\ 6 & 0.038709 & -0.061524 & 8.595223 \\ 1 & -0.200381 & -0.921179 & 7.967556 \\ 6 & 0.065339 & 4.612504 & 10.969864 \\ 6 & 3.536569 & 5.577629 & 11.853536 \\ 1 & 3.312528 & 6.657506 & 11.846987 \\ 1 & 4.287469 & 5.452962 & 12.636978 \\ 6 & -2.281541 & 1.346857 & 11.816415 \\ 1 & -2.527106 & 1.986755 & 10.957275 \\ 1 & -2.791698 & 1.741590 & 12.708178 \\ 1 & -2.700098 & 0.349861 & 11.609644 \\ 6 & 1.109962 & 5.627121 & 11.421865 \\ 1 & 0.674311 & 6.443526 & 12.016454 \\ 1 & 1.559038 & 6.079532 & 10.524875 \\ 6 & -0.560393 & 4.996265 & 9.628063 \\ 1 & 0.195159 & 5.258655 & 8.877826 \\ 1 & -1.211120 & 5.870696 & 9.771842 \\ 1 & -1.175968 & 4.173937 & 9.232578 \\ 6 & 2.210847 & 6.295223 & 14.380752 \\ 1 & 1.709495 & 7.125189 & 13.860033 \\ 1 & 3.288760 & 6.510172 & 14.385676 \\ 1 & 1.867481 & 6.304338 & 15.424560 \\ & & & \end{array}$




$\begin{array}{cccc}6 & -1.090049 & 4.501659 & 11.981315 \\ 1 & -1.866766 & 3.819703 & 11.616567 \\ 1 & -1.549419 & 5.493045 & 12.109032 \\ 1 & -0.762130 & 4.150661 & 12.966638 \\ 6 & 4.138223 & 5.184829 & 10.511380 \\ 1 & 3.428434 & 5.334979 & 9.683230 \\ 1 & 4.423818 & 4.123382 & 10.504395 \\ 1 & 5.032118 & 5.786577 & 10.293683 \\ 6 & 3.314836 & 2.919479 & 7.852532 \\ 1 & 3.826312 & 2.669942 & 8.792604 \\ 1 & 3.831652 & 3.779088 & 7.398718 \\ 1 & 3.420323 & 2.065970 & 7.164698 \\ 6 & 1.166513 & 3.672793 & 6.783184 \\ 1 & 1.204058 & 2.846595 & 6.056393 \\ 1 & 1.676539 & 4.537013 & 6.330988 \\ 1 & 0.109583 & 3.932665 & 6.936690 \\ 1 & 5.628011 & 2.855974 & 14.053711 \\ 1 & 4.192094 & 1.174028 & 14.957029 \\ 1 & 5.830559 & 0.914495 & 13.053590 \\ 1 & 2.084946 & -0.597871 & 12.116935 \\ 32 & 2.318518 & 0.547786 & 11.111269\end{array}$

$\begin{array}{lccc}\mathbf{P}(\mathbf{G e}=\mathbf{C}): & & \\ 7 & 0.882475 & 3.425997 & 10.920222 \\ 6 & -0.835379 & 1.233422 & 12.034723 \\ 1 & -0.496987 & 2.142998 & 12.549626 \\ 6 & 2.743938 & 2.291152 & 12.008124 \\ 6 & 4.008624 & 2.035396 & 12.868993 \\ 6 & -0.392473 & 0.003215 & 9.866874 \\ 1 & -1.252663 & -0.612901 & 10.137882 \\ 6 & 4.928880 & 1.049239 & 12.114820 \\ 1 & 4.463482 & 0.067242 & 11.942785 \\ 1 & 5.221695 & 1.465656 & 11.138150 \\ 6 & 0.102525 & 1.024055 & 10.837641 \\ 6 & 2.581449 & 0.115766 & 9.426329 \\ 1 & 3.553811 & 0.565069 & 9.185536 \\ 1 & 2.669413 & -0.974015 & 9.304280 \\ 6 & 3.577262 & 1.388201 & 14.201096 \\ 1 & 2.954404 & 2.082869 & 14.783734 \\ 1 & 2.994620 & 0.470437 & 14.041135 \\ 6 & 2.027808 & 3.433497 & 11.743344 \\ 6 & 1.235615 & 2.116186 & 8.928545 \\ 6 & 4.923104 & 3.214971 & 13.224753 \\ 1 & 5.317122 & 3.713380 & 12.329969 \\ 1 & 4.438436 & 3.955869 & 13.868220 \\ 6 & 1.463301 & 0.681848 & 8.481383\end{array}$




$\begin{array}{rrrc}1 & 1.797399 & 0.643253 & 7.434790 \\ 6 & 0.615752 & 2.273107 & 10.126461 \\ 6 & 2.187139 & 4.902645 & 12.238161 \\ 6 & 1.925578 & 3.220563 & 8.165648 \\ 1 & 1.870798 & 4.123344 & 8.790019 \\ 6 & 1.939147 & 4.961659 & 13.765126 \\ 1 & 0.904991 & 4.645766 & 13.961944 \\ 1 & 2.565358 & 4.205912 & 14.257721 \\ 6 & -0.764264 & 0.086235 & 13.044792 \\ 1 & -0.942305 & -0.884564 & 12.554228 \\ 1 & -1.528616 & 0.210611 & 13.826498 \\ 1 & 0.216497 & 0.036759 & 13.539698 \\ 6 & 0.213785 & -0.131147 & 8.683593 \\ 1 & -0.122042 & -0.839235 & 7.924280 \\ 6 & 0.029850 & 4.623999 & 11.026803 \\ 6 & 3.509293 & 5.624327 & 11.865740 \\ 1 & 3.285509 & 6.704075 & 11.893211 \\ 1 & 4.274485 & 5.475809 & 12.632401 \\ 6 & -2.288217 & 1.430144 & 11.580366 \\ 1 & -2.350980 & 2.076149 & 10.691451 \\ 1 & -2.898149 & 1.874647 & 12.381376 \\ 1 & -2.749820 & 0.466814 & 11.316419 \\ 6 & 1.071731 & 5.650545 & 11.468728 \\ 1 & 0.633987 & 6.457988 & 12.073520 \\ 1 & 1.501715 & 6.114778 & 10.568644 \\ 6 & -0.602974 & 4.981257 & 9.682890 \\ 1 & 0.154459 & 5.207902 & 8.921700 \\ 1 & -1.244281 & 5.866435 & 9.801905 \\ 1 & -1.226552 & 4.151825 & 9.315446 \\ 6 & 2.182832 & 6.321745 & 14.413737 \\ 1 & 1.609036 & 7.120990 & 13.920678 \\ 1 & 3.244129 & 6.606469 & 14.380506 \\ 1 & 1.880068 & 6.302088 & 15.470009 \\ 6 & -1.108229 & 4.521493 & 12.058004 \\ 1 & -1.896374 & 3.843106 & 11.714204 \\ 1 & -1.558841 & 5.516179 & 12.192616 \\ 1 & -0.756827 & 4.172794 & 13.036693 \\ 6 & 4.081991 & 5.268927 & 10.501254 \\ 1 & 3.372285 & 5.481751 & 9.687072 \\ 1 & 4.326085 & 4.198819 & 10.447254 \\ 1 & 4.997043 & 5.844012 & 10.299125 \\ 6 & 3.409443 & 2.934329 & 7.912929 \\ 1 & 3.940694 & 2.739212 & 8.855050 \\ 1 & 3.883436 & 3.800978 & 7.427494 \\ 1 & 3.548616 & 2.065509 & 7.250713 \\ 6 & 1.197233 & 3.490485 & 6.843685\end{array}$




$\begin{array}{cccc}1 & 1.222657 & 2.600946 & 6.194663 \\ 1 & 1.676124 & 4.317074 & 6.296958 \\ 1 & 0.142822 & 3.748250 & 7.016031 \\ 1 & 5.783923 & 2.821737 & 13.786279 \\ 1 & 4.459810 & 1.124892 & 14.806101 \\ 1 & 5.844455 & 0.862252 & 12.695845 \\ 1 & 2.222424 & -0.624318 & 12.226958 \\ 32 & 2.028367 & 0.585235 & 11.274394\end{array}$

\section{$\mathbf{S M}(\mathbf{P}=\mathbf{S i})$ :}

$\begin{array}{cccc}15 & 2.065426 & 0.633599 & 12.179287 \\ 7 & 0.827034 & 3.158964 & 11.149417 \\ 6 & -1.364218 & 1.145881 & 11.658662 \\ 1 & -0.906430 & 1.830741 & 12.383401 \\ 6 & 2.932641 & 2.285653 & 12.063944 \\ 6 & 4.357315 & 2.225295 & 12.701787 \\ 6 & -0.782178 & 0.242249 & 9.414857 \\ 1 & -1.570539 & -0.491978 & 9.590572 \\ 6 & 5.267659 & 1.243165 & 11.933290 \\ 1 & 4.929685 & 0.205214 & 12.025205 \\ 1 & 5.329416 & 1.501446 & 10.865166 \\ 6 & -0.529951 & 1.211891 & 10.387791 \\ 6 & 4.245604 & 1.716009 & 14.155089 \\ 1 & 3.618294 & 2.384120 & 14.764073 \\ 1 & 3.810766 & 0.707980 & 14.201622 \\ 6 & 2.123489 & 3.345118 & 11.727686 \\ 6 & 1.206116 & 2.140291 & 8.941031 \\ 6 & 5.158807 & 3.529484 & 12.754044 \\ 1 & 5.283128 & 3.974991 & 11.760175 \\ 1 & 4.726443 & 4.264280 & 13.438361 \\ 6 & 0.968867 & 1.105077 & 8.031522 \\ 1 & 1.553085 & 1.066418 & 7.109178 \\ 6 & 0.501450 & 2.164000 & 10.165578 \\ 6 & 2.234164 & 4.868890 & 12.107414 \\ 6 & 2.234578 & 3.183568 & 8.550650 \\ 1 & 2.242214 & 3.944734 & 9.336941 \\ 6 & 2.122134 & 4.956461 & 13.654376 \\ 1 & 1.189341 & 4.470263 & 13.966876 \\ 1 & 2.918603 & 4.341184 & 14.092706 \\ 6 & -1.380016 & -0.249411 & 12.295927 \\ 1 & -1.911207 & -0.976028 & 11.663051 \\ 1 & -1.910397 & -0.208661 & 13.259309 \\ 1 & -0.364614 & -0.620861 & 12.480722 \\ 6 & -0.016340 & 0.152141 & 8.259255 \\ 1 & -0.200066 & -0.639540 & 7.531125 \\ 6 & -0.047020 & 4.355886 & 11.273135\end{array}$




$\begin{array}{cccc}6 & 3.410630 & 5.770230 & 11.637296 \\ 1 & 3.027412 & 6.801170 & 11.720484 \\ 1 & 4.243779 & 5.732779 & 12.342729 \\ 6 & -2.814579 & 1.570635 & 11.385814 \\ 1 & -2.886423 & 2.539103 & 10.873682 \\ 1 & -3.383591 & 1.635213 & 12.326050 \\ 1 & -3.311614 & 0.826143 & 10.744618 \\ 6 & 0.988978 & 5.461836 & 11.431064 \\ 1 & 0.598078 & 6.327779 & 11.983510 \\ 1 & 1.266408 & 5.818143 & 10.426656 \\ 6 & -0.871228 & 4.605204 & 10.010729 \\ 1 & -0.222139 & 4.678663 & 9.127523 \\ 1 & -1.415375 & 5.554579 & 10.125107 \\ 1 & -1.601911 & 3.806897 & 9.828352 \\ 6 & 2.184554 & 6.357986 & 14.256221 \\ 1 & 1.421313 & 7.028361 & 13.833197 \\ 1 & 3.165169 & 6.830536 & 14.103156 \\ 1 & 2.010195 & 6.309342 & 15.340140 \\ 6 & -0.999762 & 4.304882 & 12.485442 \\ 1 & -1.916057 & 3.749569 & 12.256520 \\ 1 & -1.296632 & 5.326445 & 12.766692 \\ 1 & -0.529794 & 3.827824 & 13.353900 \\ 6 & 3.946755 & 5.581352 & 10.224628 \\ 1 & 3.198516 & 5.824113 & 9.455737 \\ 1 & 4.278493 & 4.549234 & 10.052547 \\ 1 & 4.806135 & 6.246911 & 10.059198 \\ 6 & 3.638375 & 2.581141 & 8.461348 \\ 1 & 3.948626 & 2.181036 & 9.436788 \\ 1 & 4.370274 & 3.339552 & 8.144184 \\ 1 & 3.668875 & 1.761043 & 7.726837 \\ 6 & 1.868264 & 3.876070 & 7.233123 \\ 1 & 1.935724 & 3.179669 & 6.383497 \\ 1 & 2.563363 & 4.706327 & 7.034827 \\ 1 & 0.844918 & 4.277551 & 7.252306 \\ 1 & 6.162366 & 3.297300 & 13.138628 \\ 1 & 5.247488 & 1.673073 & 14.610416 \\ 1 & 6.285016 & 1.292485 & 12.348979 \\ 14 & 2.686393 & -0.599261 & 10.633248 \\ 1 & 3.701185 & -0.390241 & 9.568899 \\ 1 & 2.134306 & -1.976485 & 10.604982\end{array}$

\section{$\mathbf{T S}(\mathbf{P}=\mathbf{S i})$ : \\ $\begin{array}{llll}15 & 2.071709 & 0.517852 & 11.799763\end{array}$ \\ $\begin{array}{llll}7 & 0.813537 & 3.293638 & 11.055162\end{array}$ \\ $\begin{array}{llll}6 & -1.169195 & 1.203998 & 11.845986\end{array}$ \\ $\begin{array}{lllll}1 & -0.770179 & 2.005454 & 12.479916\end{array}$}




$\begin{array}{rrrc}6 & 2.836379 & 2.251945 & 11.972995 \\ 6 & 4.207880 & 2.117482 & 12.717945 \\ 6 & -0.569080 & 0.064474 & 9.707638 \\ 1 & -1.327143 & -0.675326 & 9.966195 \\ 6 & 5.162149 & 1.176189 & 11.952065 \\ 1 & 4.797250 & 0.144080 & 11.918376 \\ 1 & 5.314094 & 1.524621 & 10.917984 \\ 6 & -0.278024 & 1.116684 & 10.618305 \\ 6 & 3.963050 & 1.512221 & 14.117378 \\ 1 & 3.318679 & 2.167653 & 14.722657 \\ 1 & 3.483094 & 0.526494 & 14.056346 \\ 6 & 2.073527 & 3.367296 & 11.716548 \\ 6 & 1.212063 & 2.118813 & 8.965659 \\ 6 & 5.034963 & 3.388852 & 12.938346 \\ 1 & 5.293446 & 3.882184 & 11.994128 \\ 1 & 4.551654 & 4.104024 & 13.608641 \\ 6 & 1.242008 & 0.834310 & 8.329901 \\ 1 & 1.797650 & 0.734953 & 7.392922 \\ 6 & 0.518522 & 2.225968 & 10.162329 \\ 6 & 2.222443 & 4.865172 & 12.171873 \\ 6 & 2.067895 & 3.225542 & 8.388329 \\ 1 & 2.013422 & 4.069606 & 9.088520 \\ 6 & 2.036674 & 4.917105 & 13.711747 \\ 1 & 1.040609 & 4.524823 & 13.955137 \\ 1 & 2.736096 & 4.209836 & 14.175504 \\ 6 & -1.221566 & -0.076473 & 12.682845 \\ 1 & -1.604009 & -0.927095 & 12.098347 \\ 1 & -1.907226 & 0.069374 & 13.530612 \\ 1 & -0.230682 & -0.341474 & 13.070730 \\ 6 & 0.195711 & -0.093842 & 8.585346 \\ 1 & 0.069420 & -0.960193 & 7.932778 \\ 6 & -0.023856 & 4.505383 & 11.161467 \\ 6 & 3.463545 & 5.703552 & 11.757458 \\ 1 & 3.140544 & 6.756216 & 11.823279 \\ 1 & 4.269559 & 5.618238 & 12.488871 \\ 6 & -2.595605 & 1.562504 & 11.394201 \\ 1 & -2.610876 & 2.398470 & 10.681358 \\ 1 & -3.218380 & 1.831198 & 12.260957 \\ 1 & -3.066773 & 0.700815 & 10.897452 \\ 6 & 1.040721 & 5.554981 & 11.457437 \\ 1 & 0.650764 & 6.397421 & 12.046495 \\ 1 & 1.393853 & 5.968043 & 10.499850 \\ 6 & -0.744754 & 4.810891 & 9.848770 \\ 1 & -0.031998 & 4.927327 & 9.021734 \\ 1 & -1.309337 & 5.748891 & 9.954069 \\ 1 & -1.452706 & 4.012266 & 9.583194\end{array}$




$\begin{array}{cccc}6 & 2.207362 & 6.290376 & 14.356352 \\ 1 & 1.544431 & 7.045138 & 13.906954 \\ 1 & 3.239034 & 6.659550 & 14.269789 \\ 1 & 1.965807 & 6.238762 & 15.427220 \\ 6 & -1.080175 & 4.461216 & 12.282793 \\ 1 & -1.942014 & 3.846215 & 12.002064 \\ 1 & -1.450708 & 5.480024 & 12.470822 \\ 1 & -0.673345 & 4.065862 & 13.221131 \\ 6 & 4.020851 & 5.455402 & 10.363298 \\ 1 & 3.304311 & 5.723742 & 9.572163 \\ 1 & 4.285532 & 4.399313 & 10.223887 \\ 1 & 4.924649 & 6.059767 & 10.198566 \\ 6 & 3.540194 & 2.834007 & 8.244877 \\ 1 & 3.960595 & 2.512924 & 9.208595 \\ 1 & 4.124546 & 3.692722 & 7.881850 \\ 1 & 3.670770 & 2.013449 & 7.522011 \\ 6 & 1.509141 & 3.679748 & 7.033535 \\ 1 & 1.602523 & 2.878438 & 6.284180 \\ 1 & 2.066472 & 4.552501 & 6.660645 \\ 1 & 0.445412 & 3.947555 & 7.102004 \\ 1 & 5.978671 & 3.097062 & 13.422202 \\ 1 & 4.923172 & 1.391842 & 14.643657 \\ 1 & 6.141556 & 1.167204 & 12.453306 \\ 14 & 2.714949 & -0.222948 & 9.897551 \\ 1 & 3.971958 & 0.146699 & 9.203595 \\ 1 & 2.443351 & -1.667700 & 9.674485\end{array}$

$\begin{array}{lccc}\mathbf{P}(\mathbf{P}=\mathbf{S i}): \\ 15 & 2.043777 & 0.537095 & 11.530654 \\ 7 & 0.928135 & 3.424715 & 10.878538 \\ 6 & -0.705480 & 1.190211 & 11.935057 \\ 1 & -0.394823 & 2.074451 & 12.506615 \\ 6 & 2.689236 & 2.223617 & 12.072566 \\ 6 & 3.884837 & 1.969741 & 13.043495 \\ 6 & -0.107185 & -0.082276 & 9.862698 \\ 1 & -0.863889 & -0.794385 & 10.195510 \\ 6 & 4.831060 & 0.892935 & 12.462811 \\ 1 & 4.343408 & -0.082751 & 12.329334 \\ 1 & 5.245681 & 1.216371 & 11.495676 \\ 6 & 0.336544 & 1.001211 & 10.807914 \\ 6 & 3.328758 & 1.440475 & 14.381453 \\ 1 & 2.688738 & 2.190645 & 14.868501 \\ 1 & 2.729947 & 0.531693 & 14.226987 \\ 6 & 2.020589 & 3.386582 & 11.768672 \\ 6 & 1.234552 & 2.194939 & 8.820649 \\ 6 & 4.818887 & 3.145205 & 13.356734\end{array}$




$\begin{array}{rrrc}1 & 5.294166 & 3.535534 & 12.447003 \\ 1 & 4.327128 & 3.964624 & 13.886857 \\ 6 & 1.511505 & 0.810862 & 8.300056 \\ 1 & 1.782570 & 0.792609 & 7.237157 \\ 6 & 0.703199 & 2.283648 & 10.064461 \\ 6 & 2.232862 & 4.862128 & 12.237451 \\ 6 & 1.730600 & 3.369544 & 8.008916 \\ 1 & 1.693511 & 4.245541 & 8.671010 \\ 6 & 2.000904 & 5.016987 & 13.761469 \\ 1 & 0.930869 & 4.872041 & 13.961943 \\ 1 & 2.504423 & 4.205687 & 14.300989 \\ 6 & -0.769471 & 0.018026 & 12.915883 \\ 1 & -0.979219 & -0.931775 & 12.399076 \\ 1 & -1.577547 & 0.183689 & 13.643866 \\ 1 & 0.171051 & -0.105249 & 13.468645 \\ 6 & 0.433379 & -0.165981 & 8.644976 \\ 1 & 0.153091 & -0.947185 & 7.935899 \\ 6 & 0.062391 & 4.607801 & 11.059166 \\ 6 & 3.575677 & 5.507111 & 11.789634 \\ 1 & 3.395046 & 6.594270 & 11.753628 \\ 1 & 4.358794 & 5.376175 & 12.540047 \\ 6 & -2.094266 & 1.441534 & 11.337412 \\ 1 & -2.057734 & 2.168885 & 10.512765 \\ 1 & -2.789786 & 1.814857 & 12.103857 \\ 1 & -2.520120 & 0.510930 & 10.933756 \\ 6 & 1.127376 & 5.625661 & 11.464084 \\ 1 & 0.719213 & 6.455179 & 12.060305 \\ 1 & 1.552330 & 6.058055 & 10.545348 \\ 6 & -0.660579 & 4.996893 & 9.772844 \\ 1 & 0.033776 & 5.305642 & 8.982137 \\ 1 & -1.337616 & 5.838611 & 9.977361 \\ 1 & -1.265131 & 4.156674 & 9.398469 \\ 6 & 2.428541 & 6.360111 & 14.346939 \\ 1 & 1.966773 & 7.204067 & 13.812004 \\ 1 & 3.518080 & 6.498393 & 14.307291 \\ 1 & 2.125502 & 6.431624 & 15.400805 \\ 6 & -1.004391 & 4.462816 & 12.161792 \\ 1 & -1.838434 & 3.834603 & 11.829638 \\ 1 & -1.410888 & 5.456870 & 12.401067 \\ 1 & -0.597078 & 4.030569 & 13.083666 \\ 6 & 4.092694 & 5.040995 & 10.436350 \\ 1 & 3.355055 & 5.206427 & 9.636878 \\ 1 & 4.317254 & 3.964078 & 10.452335 \\ 1 & 5.008299 & 5.583534 & 10.160716 \\ 6 & 3.174274 & 3.229633 & 7.517068 \\ 1 & 3.878262 & 3.114576 & 8.350590\end{array}$




$\begin{array}{cccc}1 & 3.461174 & 4.130402 & 6.953498 \\ 1 & 3.297126 & 2.368737 & 6.841661 \\ 6 & 0.791280 & 3.608016 & 6.819390 \\ 1 & 0.817506 & 2.750885 & 6.128212 \\ 1 & 1.095649 & 4.502278 & 6.254428 \\ 1 & -0.248379 & 3.739267 & 7.148767 \\ 1 & 5.620558 & 2.775400 & 14.013152 \\ 1 & 4.155011 & 1.193122 & 15.067016 \\ 1 & 5.670321 & 0.737641 & 13.156715 \\ 14 & 2.972093 & 0.241559 & 9.462961 \\ 1 & 4.215231 & 1.037176 & 9.278733 \\ 1 & 3.294287 & -1.190656 & 9.204472\end{array}$

\section{$\mathbf{S M}(\mathbf{P}=\mathbf{G e})$ :}

$\begin{array}{lllll}15 & 2.059491 & 0.634920 & 12.215685\end{array}$

$\begin{array}{llll}7 & 0.827716 & 3.156351 & 11.151658\end{array}$

$\begin{array}{lllll}6 & -1.368406 & 1.143287 & 11.650423\end{array}$

$\begin{array}{llll}1 & -0.909232 & 1.822581 & 12.379381\end{array}$

$\begin{array}{llll}6 & 2.931339 & 2.281530 & 12.073464\end{array}$

$\begin{array}{lllll}6 & 4.360578 & 2.229848 & 12.703692\end{array}$

$\begin{array}{llll}6 & -0.788890 & 0.253061 & 9.400989\end{array}$

$\begin{array}{llll}1 & -1.581210 & -0.478223 & 9.571099\end{array}$

$\begin{array}{llll}6 & 5.263282 & 1.232649 & 11.945289\end{array}$

$\begin{array}{lllll}1 & 4.924544 & 0.197505 & 12.061728\end{array}$

$\begin{array}{llll}1 & 5.316119 & 1.469671 & 10.871732\end{array}$

$\begin{array}{llll}6 & -0.533092 & 1.215148 & 10.380413\end{array}$

$\begin{array}{llll}6 & 4.261193 & 1.743223 & 14.165511\end{array}$

$\begin{array}{llll}1 & 3.640765 & 2.420844 & 14.770851\end{array}$

$\begin{array}{lllll}1 & 3.825291 & 0.736827 & 14.230101\end{array}$

$\begin{array}{lllll}6 & 2.123658 & 3.341867 & 11.731418\end{array}$

$\begin{array}{llll}6 & 1.209066 & 2.144155 & 8.941761\end{array}$

$\begin{array}{llll}6 & 5.164322 & 3.533372 & 12.728363\end{array}$

$\begin{array}{lllll}1 & 5.272226 & 3.968874 & 11.728068\end{array}$

$\begin{array}{llll}1 & 4.742819 & 4.274801 & 13.412176\end{array}$

$\begin{array}{lllll}6 & 0.969673 & 1.114291 & 8.026149\end{array}$

$\begin{array}{lllll}1 & 1.555235 & 1.078476 & 7.104498\end{array}$

$\begin{array}{llll}6 & 0.502323 & 2.164819 & 10.165088\end{array}$

$\begin{array}{llll}6 & 2.233925 & 4.867621 & 12.109018\end{array}$

$\begin{array}{lllll}6 & 2.240384 & 3.186905 & 8.557729\end{array}$

$\begin{array}{lllll}1 & 2.245437 & 3.945918 & 9.345948\end{array}$

$\begin{array}{lllll}6 & 2.130568 & 4.954876 & 13.656569\end{array}$

$\begin{array}{lllll}1 & 1.205141 & 4.458491 & 13.975345\end{array}$

$\begin{array}{lllll}1 & 2.936766 & 4.348402 & 14.089167\end{array}$

$\begin{array}{llll}6 & -1.387311 & -0.255733 & 12.279538\end{array}$

$\begin{array}{lllll}1 & -1.914072 & -0.979194 & 11.639327\end{array}$

$\begin{array}{llll}1 & -1.923870 & -0.220488 & 13.239711\end{array}$ 


$\begin{array}{rrrc}1 & -0.372995 & -0.627417 & 12.469589 \\ 6 & -0.021221 & 0.165285 & 8.245986 \\ 1 & -0.208126 & -0.620799 & 7.512586 \\ 6 & -0.049144 & 4.350357 & 11.281859 \\ 6 & 3.404445 & 5.775349 & 11.634660 \\ 1 & 3.017026 & 6.803938 & 11.726924 \\ 1 & 4.242847 & 5.737848 & 12.333764 \\ 6 & -2.818299 & 1.571528 & 11.380411 \\ 1 & -2.889747 & 2.544105 & 10.876217 \\ 1 & -3.387161 & 1.629132 & 12.321169 \\ 1 & -3.316198 & 0.832501 & 10.733572 \\ 6 & 0.984570 & 5.458295 & 11.438608 \\ 1 & 0.593225 & 6.322124 & 11.993930 \\ 1 & 1.257149 & 5.817404 & 10.433937 \\ 6 & -0.877885 & 4.601600 & 10.022799 \\ 1 & -0.231339 & 4.677488 & 9.137810 \\ 1 & -1.422395 & 5.550306 & 10.141019 \\ 1 & -1.608339 & 3.803233 & 9.840386 \\ 6 & 2.183041 & 6.356617 & 14.258867 \\ 1 & 1.410245 & 7.019872 & 13.842105 \\ 1 & 3.157887 & 6.838917 & 14.099627 \\ 1 & 2.016779 & 6.305196 & 15.343943 \\ 6 & -0.995860 & 4.294525 & 12.498633 \\ 1 & -1.912865 & 3.738909 & 12.273345 \\ 1 & -1.292270 & 5.314878 & 12.784708 \\ 1 & -0.520065 & 3.815465 & 13.362924 \\ 6 & 3.931954 & 5.600377 & 10.216905 \\ 1 & 3.175680 & 5.839919 & 9.454948 \\ 1 & 4.274526 & 4.573589 & 10.035261 \\ 1 & 4.782639 & 6.276617 & 10.049962 \\ 6 & 3.643859 & 2.583244 & 8.472888 \\ 1 & 3.951560 & 2.186163 & 9.450500 \\ 1 & 4.377253 & 3.339982 & 8.155257 \\ 1 & 3.675691 & 1.761039 & 7.740698 \\ 6 & 1.879424 & 3.883806 & 7.241146 \\ 1 & 1.948769 & 3.190370 & 6.389241 \\ 1 & 2.576269 & 4.713823 & 7.048113 \\ 1 & 0.856531 & 4.286619 & 7.258722 \\ 1 & 6.173956 & 3.305636 & 13.099604 \\ 1 & 5.267323 & 1.705988 & 14.612032 \\ 1 & 6.284395 & 1.288148 & 12.350803 \\ 32 & 2.674981 & -0.635428 & 10.608074 \\ 1 & 3.725964 & -0.426361 & 9.498980 \\ 1 & 2.107412 & -2.069055 & 10.572877\end{array}$

\section{TS $(\mathbf{P}=\mathbf{G e})$ :}




$\begin{array}{rrrc}15 & 2.056305 & 0.523596 & 11.798840 \\ 7 & 0.820382 & 3.302241 & 11.040925 \\ 6 & -1.138082 & 1.198370 & 11.861908 \\ 1 & -0.741990 & 2.004191 & 12.492431 \\ 6 & 2.827724 & 2.250803 & 11.980997 \\ 6 & 4.191098 & 2.115727 & 12.741803 \\ 6 & -0.551926 & 0.056645 & 9.721680 \\ 1 & -1.297536 & -0.690360 & 9.995361 \\ 6 & 5.155571 & 1.177130 & 11.985773 \\ 1 & 4.796734 & 0.142802 & 11.954031 \\ 1 & 5.313817 & 1.523591 & 10.951922 \\ 6 & -0.244415 & 1.105627 & 10.634191 \\ 6 & 3.930873 & 1.507803 & 14.137227 \\ 1 & 3.283166 & 2.163890 & 14.738196 \\ 1 & 3.447926 & 0.523886 & 14.068816 \\ 6 & 2.068447 & 3.368730 & 11.718978 \\ 6 & 1.191241 & 2.130520 & 8.944428 \\ 6 & 5.015720 & 3.386866 & 12.973487 \\ 1 & 5.292245 & 3.875899 & 12.032078 \\ 1 & 4.520958 & 4.105549 & 13.631285 \\ 6 & 1.205343 & 0.854306 & 8.291207 \\ 1 & 1.734885 & 0.766765 & 7.338363 \\ 6 & 0.521925 & 2.229015 & 10.153634 \\ 6 & 2.221000 & 4.866302 & 12.174784 \\ 6 & 2.041128 & 3.240421 & 8.363236 \\ 1 & 1.989026 & 4.083077 & 9.065167 \\ 6 & 2.028225 & 4.925768 & 13.713553 \\ 1 & 1.019718 & 4.563197 & 13.950782 \\ 1 & 2.703906 & 4.201119 & 14.185345 \\ 6 & -1.194214 & -0.076539 & 12.706839 \\ 1 & -1.564187 & -0.933148 & 12.123036 \\ 1 & -1.892147 & 0.070975 & 13.544257 \\ 1 & -0.208275 & -0.335405 & 13.110799 \\ 6 & 0.175371 & -0.083439 & 8.572730 \\ 1 & 0.028368 & -0.941056 & 7.912827 \\ 6 & -0.019606 & 4.511740 & 11.152371 \\ 6 & 3.467833 & 5.695769 & 11.758303 \\ 1 & 3.149476 & 6.750383 & 11.814134 \\ 1 & 4.271358 & 5.613407 & 12.492516 \\ 6 & -2.563989 & 1.552340 & 11.404600 \\ 1 & -2.577298 & 2.376517 & 10.678115 \\ 1 & -3.187398 & 1.834922 & 12.266507 \\ 1 & -3.035562 & 0.683723 & 10.920599 \\ 6 & 1.043914 & 5.560215 & 11.456061 \\ 1 & 0.652431 & 6.401216 & 12.046433 \\ 1 & 1.401500 & 5.975425 & 10.501039\end{array}$




$\begin{array}{cccc}6 & -0.740955 & 4.823185 & 9.841498 \\ 1 & -0.029205 & 4.950855 & 9.015426 \\ 1 & -1.311200 & 5.756869 & 9.954341 \\ 1 & -1.443828 & 4.022194 & 9.569719 \\ 6 & 2.230535 & 6.296586 & 14.354506 \\ 1 & 1.597420 & 7.068128 & 13.890695 \\ 1 & 3.273869 & 6.634472 & 14.281294 \\ 1 & 1.971701 & 6.258130 & 15.421892 \\ 6 & -1.077146 & 4.458396 & 12.272230 \\ 1 & -1.936956 & 3.843440 & 11.985851 \\ 1 & -1.449727 & 5.475239 & 12.466694 \\ 1 & -0.671649 & 4.056848 & 13.208444 \\ 6 & 4.027791 & 5.431715 & 10.368368 \\ 1 & 3.312872 & 5.691606 & 9.573084 \\ 1 & 4.291550 & 4.373666 & 10.241485 \\ 1 & 4.932578 & 6.033183 & 10.198488 \\ 6 & 3.513162 & 2.847844 & 8.218565 \\ 1 & 3.932126 & 2.523350 & 9.182103 \\ 1 & 4.100397 & 3.706095 & 7.858862 \\ 1 & 3.642628 & 2.028623 & 7.493815 \\ 6 & 1.480318 & 3.698500 & 7.011021 \\ 1 & 1.573767 & 2.900960 & 6.257711 \\ 1 & 2.035315 & 4.573766 & 6.640552 \\ 1 & 0.416205 & 3.964251 & 7.082377 \\ 1 & 5.949855 & 3.095511 & 13.475818 \\ 1 & 4.885168 & 1.382088 & 14.672814 \\ 1 & 6.131293 & 1.173449 & 12.494134 \\ 32 & 2.777677 & -0.263451 & 9.860314 \\ 1 & 4.088468 & 0.125642 & 9.150924 \\ 1 & 2.485551 & -1.760353 & 9.608460\end{array}$

\begin{tabular}{lrrr}
\multicolumn{4}{l}{$\mathbf{P}(\mathbf{P}=\mathbf{G e}):$} \\
15 & 2.047644 & 0.534549 & 11.550388 \\
7 & 0.937802 & 3.419706 & 10.878716 \\
6 & -0.700329 & 1.185723 & 11.931252 \\
1 & -0.390465 & 2.068645 & 12.505169 \\
6 & 2.689626 & 2.220425 & 12.089621 \\
6 & 3.876499 & 1.971460 & 13.073601 \\
6 & -0.096738 & -0.087453 & 9.863061 \\
1 & -0.838057 & -0.810942 & 10.205992 \\
6 & 4.821763 & 0.880202 & 12.518487 \\
1 & 4.331179 & -0.095800 & 12.401207 \\
1 & 5.244592 & 1.183287 & 11.548209 \\
6 & 0.346858 & 0.996518 & 10.807166 \\
6 & 3.306302 & 1.462802 & 14.413494 \\
1 & 2.667031 & 2.222095 & 14.886930
\end{tabular}




$\begin{array}{rrrc}1 & 2.702677 & 0.556128 & 14.264452 \\ 6 & 2.024083 & 3.382876 & 11.775602 \\ 6 & 1.221348 & 2.197118 & 8.810653 \\ 6 & 4.815776 & 3.145660 & 13.375974 \\ 1 & 5.297490 & 3.519971 & 12.462737 \\ 1 & 4.326871 & 3.975564 & 13.891884 \\ 6 & 1.480662 & 0.822838 & 8.267000 \\ 1 & 1.729952 & 0.813658 & 7.199512 \\ 6 & 0.708958 & 2.278763 & 10.063711 \\ 6 & 2.240161 & 4.860886 & 12.235924 \\ 6 & 1.710013 & 3.377424 & 8.001615 \\ 1 & 1.682399 & 4.249316 & 8.669282 \\ 6 & 2.010116 & 5.030618 & 13.758839 \\ 1 & 0.938832 & 4.897625 & 13.960866 \\ 1 & 2.506305 & 4.220055 & 14.305902 \\ 6 & -0.772916 & 0.013335 & 12.911487 \\ 1 & -0.986655 & -0.935425 & 12.394525 \\ 1 & -1.582433 & 0.183478 & 13.636815 \\ 1 & 0.164706 & -0.115120 & 13.467965 \\ 6 & 0.421645 & -0.161098 & 8.634499 \\ 1 & 0.134269 & -0.944934 & 7.930959 \\ 6 & 0.070735 & 4.602813 & 11.060549 \\ 6 & 3.584960 & 5.496723 & 11.780291 \\ 1 & 3.408774 & 6.584304 & 11.736692 \\ 1 & 4.369391 & 5.367869 & 12.529631 \\ 6 & -2.085609 & 1.440726 & 11.327419 \\ 1 & -2.044235 & 2.172333 & 10.506894 \\ 1 & -2.784976 & 1.810207 & 12.092283 \\ 1 & -2.509051 & 0.512365 & 10.916168 \\ 6 & 1.136219 & 5.622321 & 11.458621 \\ 1 & 0.729256 & 6.455784 & 12.050206 \\ 1 & 1.561046 & 6.048697 & 10.536956 \\ 6 & -0.661139 & 4.990978 & 9.779170 \\ 1 & 0.026170 & 5.306846 & 8.985277 \\ 1 & -1.341615 & 5.828130 & 9.990815 \\ 1 & -1.263213 & 4.148703 & 9.405708 \\ 6 & 2.449711 & 6.375173 & 14.332088 \\ 1 & 1.995248 & 7.218250 & 13.789560 \\ 1 & 3.540423 & 6.503786 & 14.291295 \\ 1 & 2.147346 & 6.458838 & 15.385241 \\ 6 & -0.989036 & 4.456587 & 12.169881 \\ 1 & -1.825416 & 3.829154 & 11.841995 \\ 1 & -1.393633 & 5.450347 & 12.413653 \\ 1 & -0.575668 & 4.022404 & 13.088163 \\ 6 & 4.097085 & 5.019191 & 10.429032 \\ 1 & 3.357748 & 5.181354 & 9.630562\end{array}$




$\begin{array}{cccc}1 & 4.319094 & 3.941794 & 10.451806 \\ 1 & 5.013429 & 5.557308 & 10.147314 \\ 6 & 3.148026 & 3.236226 & 7.493639 \\ 1 & 3.860959 & 3.115492 & 8.318795 \\ 1 & 3.431819 & 4.138176 & 6.930409 \\ 1 & 3.260613 & 2.377266 & 6.813951 \\ 6 & 0.760232 & 3.626586 & 6.822454 \\ 1 & 0.782609 & 2.777166 & 6.121831 \\ 1 & 1.058430 & 4.527392 & 6.264619 \\ 1 & -0.277080 & 3.752329 & 7.161027 \\ 1 & 5.612004 & 2.780526 & 14.041566 \\ 1 & 4.125197 & 1.217708 & 15.108657 \\ 1 & 5.655968 & 0.735553 & 13.220653 \\ 32 & 3.039885 & 0.214493 & 9.441589 \\ 1 & 4.327997 & 1.048782 & 9.244169 \\ 1 & 3.356200 & -1.275821 & 9.154755\end{array}$




\section{References}

S1. Niehues, M.; Kehr, G.; Erker, G.; Wibbeling, B.; Fröhlich, R.; Blacque, O.; Berke, H. J. Organomet. Chem. 2002, 663, 192.

S2. Liu, L. L.; Cao, L. L.; Zhou, J.; Stephan, D. W. Angew. Chem., Int. Ed. 2019, 58, 273.

S3. Gaussian 09, Revision B.01: Frisch, M. J.; Trucks, G. W.; Schlegel, H. B.; Scuseria, G. E.; Robb, M. A.; Cheeseman, J. R.; Scalmani, G.; Barone, V.; Mennucci, B.; Petersson, G. A.; Nakatsuji, H.; Caricato, M.; Li, X.; Hratchian, H. P.; Izmaylov, A. F.; Bloino, J.; Zheng, G.; Sonnenberg, J. L.; Hada, M.; Ehara, M.; Toyota, K.; Fukuda, R.; Hasegawa, J.; Ishida, M.; Nakajima, T.; Honda, Y.; Kitao, O.; Nakai, H.; Vreven, T.; Montgomery, J. A., Jr.; Peralta, J. E.; Ogliaro, F.; Bearpark, M.; Heyd, J. J.; Brothers, E.; Kudin, K. N.; Staroverov, V. N.; Keith, T.; Kobayashi, R.; Normand, J.; Raghavachari, K.; Rendell, A.; Burant, J. C.; Iyengar, S. S.; Tomasi, J.; Cossi, M.; Rega, N.; Millam, J. M.; Klene, M.; Knox, J. E.; Cross, J. B.; Bakken, V.; Adamo, C.; Jaramillo, J.; Gomperts, R.; Stratmann, R. E.; Yazyev, O.; Austin, A. J.; Cammi, R.; Pomelli, C.; Ochterski, J. W.; Martin, R. L.; Morokuma, K.; Zakrzewski, V. G.; Voth, G. A.; Salvador, P.; Dannenberg, J. J.; Dapprich, S.; Daniels, A. D.; Farkas, O.; Foresman, J. B.; Ortiz, J. V.; Cioslowski, J.; and Fox, D. J. Gaussian, Inc., Wallingford, CT, 2010.

S4. Zhao, Y.; Truhlar, D. G. Theor. Chem. Acc. 2008, 120, 215.

S5. Bondi, A. J. Phys. Chem. 1964, 68, 441.

S6. Glendening, E. D.; Badenhoop, J. K.; Reed, A. E.; Carpenter, J. E.; Bohmann, J. A.; Morales, C. M.; Landis, C. R.; Weinhold, F. NBO 6.0, University of Wisconsin: Madison, WI, 2013.

S7. Legault, C. Y. CYLview, 1.0b; Université de Sherbrooke: Sherbrooke, Quebec, Canada, 2009; www.cylview.org.

S8. Andrienko, G. A. ChemCraft, http://www.chemcraftprog.com.

S9. Knizia, G. J. Chem. Theory Comput. 2013, 9, 4834. 